\title{
WestVirginiaUniversity
}

THE RESEARCH REPOSITORY @ WVU

Graduate Theses, Dissertations, and Problem Reports

2021

\section{Heterogeneous Generalizations of Vertex Coloring}

\author{
Lucian Ciletti Mazza
}

Imazza@mix.wvu.edu

Follow this and additional works at: https://researchrepository.wvu.edu/etd

Part of the Discrete Mathematics and Combinatorics Commons

\section{Recommended Citation}

Mazza, Lucian Ciletti, "Heterogeneous Generalizations of Vertex Coloring" (2021). Graduate Theses, Dissertations, and Problem Reports. 8325.

https://researchrepository.wvu.edu/etd/8325

This Thesis is protected by copyright and/or related rights. It has been brought to you by the The Research Repository @ WVU with permission from the rights-holder(s). You are free to use this Thesis in any way that is permitted by the copyright and related rights legislation that applies to your use. For other uses you must obtain permission from the rights-holder(s) directly, unless additional rights are indicated by a Creative Commons license in the record and/ or on the work itself. This Thesis has been accepted for inclusion in WVU Graduate Theses, Dissertations, and Problem Reports collection by an authorized administrator of The Research Repository @ WVU. For more information, please contact researchrepository@mail.wvu.edu. 
Graduate Theses, Dissertations, and Problem Reports

2021

Heterogeneous Generalizations of Vertex Coloring

Lucian Ciletti Mazza

Follow this and additional works at: https://researchrepository.wvu.edu/etd

Part of the Discrete Mathematics and Combinatorics Commons 


\title{
Heterogeneous Generalizations of Vertex Coloring
}

\author{
Lucian Mazza
}

Dissertation submitted to the Eberly College of Arts and Sciences at West Virginia University in partial fulfillment of the requirements for the degree of Doctor of Philosophy in Mathematics

\author{
Hong-Jian Lai, Ph.D., Chair \\ John Goldwasser, Ph.D. \\ Guodong Guo, Ph.D. \\ Rong Luo, Ph.D. \\ Jerzy Wojciechowski, Ph.D.
}

Morgantown, West Virginia 2021

Keywords: Vertex Coloring, Group Coloring, Group List Coloring, DP Coloring Copyright 2021 Lucian Mazza 


\section{Abstract}

\section{Heterogeneous Generalizations of Vertex Coloring}

\section{Lucian Mazza}

This dissertation proves a collection of results in some heterogeneous generalizations of vertex coloring, i.e. generalizations in which the relationship between the colors of two adjacent vertices may differ throughout the graph. For the most part, the results are from group coloring, group list coloring, and DP coloring. The main results are as follows: a group list coloring analogue of Brooks' Theorem for multigraphs, a result linking group structure (rather than only group size) with group coloring, some results involving coloring edge-disjoint unions, and an examination of the relationship between the group and DP coloring numbers of a multigraph and its simplification. 


\section{Acknowledgments}

Many thanks to all of the wonderful people of the WVU Math Department, and my PhD Committee in particular, especially my supervisor Dr. Hong-Jian Lai. I must also thank my family, who have been there for me in even the most difficult of times. 


\section{Contents}

1 Definitions and Notation $\quad 1$

1.1 Graph Theory and Group Theory Notation . . . . . . . . . . . . . 1

1.2 Coloring Definitions and Properties . . . . . . . . . . . 3

1.2.1 Group Coloring ................ 3

1.2.2 Group List Coloring . . . . . . . . . . . . . 6

1.2.3 DP Coloring . . . . . . . . . . . . . . . . . 8

1.2.4 $S_{k}$ Coloring ................. 11

2 Group Coloring $\quad 12$

2.1 Previous Results and Immediate Consequences . . . . . . . . . . . 12

2.2 Group Coloring and Group Structure . . . . . . . . . . . . . . 13

2.2.1 Motivation .................. . . 13

2.2.2 The Snarl . . . . . . . . . . . . . . . 14

2.2.3 Counterexamples ................... 19

2.2.4 Further Questions . . . . . . . . . . . . 23

2.3 Group Coloring and Disjoint Unions . . . . . . . . . . . . 24

2.3.1 Motivation .................. 24

2.3.2 A Partial Analogue of Burr's Theorem . . . . . . . . 25

2.3.3 Forests and Arboricity . . . . . . . . . . . . . . 27

2.3.4 Analysis .................... . . 28

2.4 Group Chromatic Number of Multigraphs . . . . . . . . . . . . 30

2.4.1 Motivation ................. 30

2.4.2 A Bound from Multiplicity . . . . . . . . . . 30 
2.4 .3 Coloring Wheels . . . . . . . . . . . . . . . . 32

3 Group List Coloring $\quad 37$

3.1 An Analogue of Brooks' Theorem . . . . . . . . . . . . . . 37

3.1 .1 Motivation . . . . . . . . . . . . . . . . 37

3.1 .2 Coloring Irregular Cycles . . . . . . . . . . . . . . 38

3.1 .3 Coloring Regular Cycles . . . . . . . . . . . . . . . . 39

3.1.4 D-Group Choosability and Brooks' Theorem . . . . . . . . 44

4 DP Coloring and $S_{k}$ Coloring 48

4.1 DP Coloring and Disjoint Unions . . . . . . . . . . . . 48

4.1 .1 Motivation . . . . . . . . . . . . . . . 48

4.1.2 A Partial DP Coloring Analogue of Burr's Theorem . . . . 49

4.1 .3 Forests and Arboricity . . . . . . . . . . . . . 51

4.1 .4 Analysis . . . . . . . . . . . . . . . . 53

4.2 Comparing $\chi_{g}(G)$ and $\chi_{D P}(G) \ldots \ldots \ldots \ldots \ldots \ldots$

4.2 .1 Motivation . . . . . . . . . . . . . . . . 54

$4.2 .2 \quad$ Halin Graphs . . . . . . . . . . . . . . . . . . 54

4.2.3 An Example where $\chi_{g}(G)<\chi_{D P}(G) \ldots \ldots \ldots \ldots$

4.3 DP Chromatic Number of Multigraphs . . . . . . . . . . . . 59

4.3 .1 Motivation . . . . . . . . . . . . . . . . . . 59

4.3 .2 A Bound From Multiplicity . . . . . . . . . . . . 60

4.3 .3 Coloring Wheels . . . . . . . . . . . . . . . . 61

5 Appendices $\quad 66$

5.1 Appendix: Proof of Lemma 2.2.16 . . . . . . . . . . . 66 


\section{Chapter 1}

\section{Definitions and Notation}

\subsection{Graph Theory and Group Theory Notation}

Graphs in this dissertation are finite and loopless with multiple parallel edges permitted, unless otherwise stated. We follow Bondy and Murty [2] for undefined terms and notation in graph theory. Thus $\chi(G), \chi_{L}(G)$, and $\delta(G)$ denote the chromatic number, the list chromatic number, and the minimum degree of a graph $G$. For a positive integer $n$, let $C_{n}$ and $K_{n}$ denote the cycle of order $n$ and the complete graph of order $n$, respectively. Let $G$ be a graph and let $X$ be a set of edges not in $E(G)$ and with ends in $V(G)$, then $G \cup X$ denotes the graph (possibly with multiple edges) with vertex set $V(G)$ and edges set $E(G) \cup X$. Given any graph $G$, a block $B$ of $G$ is a maximal 2-connected subgraph of $G$.

Given a graph $G$, define an equivalence relation " " on $E(G)$ such that $e_{1} \sim e_{2}$ if and only if either $e_{1}=e_{2}$ or both $e_{1}$ and $e_{2}$ is a cycle in $G$. Two or more edges in the same equivalence class are called parallel edges. For $u, v \in V(G)$, let [uv] denote the set of parallel edges between $u$ and $v$ and let $m_{G}(u, v)$ or $|u v|$ denote $|[u v]|$. The multiplicity of $G$ (written $\mu(G)$ ) is defined as the cardinality of the largest set of parallel edges in $G$. For a graph $H$ and a positive integer $k$, we define $k H$ to be the graph obtained by replacing each edge of $H$ by a class of $k$ parallel edges joining the same pair of end vertices. Inversely, given a multigraph $G$, we define the simplification of $G$ (denoted $\hat{G}$ ) to be the simple graph formed 
by removing all but one edge from each equivalence class of parallel edges.

For $V_{1}, V_{2} \subseteq V(G)$ and $V_{1} \cap V_{2}=\varnothing$, let $G\left[V_{1}\right]$ denote the subgraph induced by $V_{1}$ and let $E_{G}\left[V_{1}, V_{2}\right]$ denote the set of edges between $V_{1}$ and $V_{2}$. We abbreviate $E_{G}\left[\{u\}, V_{2}\right]$ to $E_{G}\left[u, V_{2}\right]$, and omit the subscript when it is clear from context. Likewise, in a directed graph, we write $E^{+}(v)$ and $E^{-}(v)$ to denote the sets of arc entering and leaving $v$, respectively.

Define $\bar{\delta}(G)=\max \{\delta(H)$ : for any subgraph $H$ of $G\}$. $\bar{\delta}(G)$ is equivalent to the degeneracy of $G$.

Groups are not assumed to be abelian unless so specified. We follow Hungerford [12] for undefined terms and notation in group theory. $\Gamma$ denotes a multiplicative group with identity $1_{\Gamma}, A$ denotes specifically an abelian group, and for a subset $F \subseteq \Gamma$, define $F^{-1}=\left\{x^{-1}: x \in F\right\}$. For a collection $\Gamma_{1}, \Gamma_{2} \ldots \Gamma_{k}$ of groups, we define the direct product of these groups, denoted by $\left(\Gamma_{1} \times \Gamma_{2} \times \ldots \times \Gamma_{k}\right)$, as a group whose elements are the Cartesian product of the elements of $\Gamma_{1}, \Gamma_{2} \ldots \Gamma_{k}$ and whose operation is given by $\left(a_{1}, a_{2} \ldots a_{k}\right) \bullet\left(b_{1}, b_{2} \ldots b_{k}\right)=\left(a_{1} \bullet \bullet_{1} b_{1}, a_{2} \bullet{ }_{2} b_{2} \ldots a_{k} \bullet{ }_{k} b_{k}\right)$, where $a_{i}, b_{i} \in \Gamma_{i}$ and $\bullet_{i}$ is the group operation for $\Gamma_{i}$. In general, we define $[k]=\{1,2,3 \ldots k\}$, and let $S_{k}$ denote the symmetric group on $k$ letters, also viewed as the set of all permutations on $[k]$. 


\subsection{Coloring Definitions and Properties}

In this section, definitions and basic properties are given for various generalizations of vertex coloring. Some previous results are also given, especially those that will be referenced to prove new results in this dissertation. A more comprehensive look at the current state of research in each generalization can be found at the beginning of its corresponding chapter.

\subsubsection{Group Coloring}

Group coloring of a graph was introduced in [14] for abelian group as the dual concept of group-connectivity of graphs. In [26], the group coloring problem is relaxed to arbitrary groups. Let $F(G, \Gamma)=\{f: E(G) \rightarrow \Gamma\}$ denote the set of all mappings from $E(G)$ to $\Gamma$. Fix an orientation $D$ of $G$. For an $f \in F(G, \Gamma)$, a mapping $c: V(G) \rightarrow \Gamma$ is a $(\Gamma, f)$-coloring if $c(u) c(v)^{-1} \neq f(e)$ for any $\operatorname{arc} e=(u, v) \in A(D)$. If for any $f \in F(G, \Gamma), G$ always admits a $(\Gamma, f)$ coloring, then $G$ is $\Gamma$-colorable. It is indicated in [23] that the question of whether $G$ is $\Gamma$-colorable is independent of the choice of the orientation of $G$. The group chromatic number, denoted $\chi_{g}(G)$, is the minimum $m$ such that $G$ is $\Gamma$-colorable for any group $\Gamma$ with $|\Gamma| \geq m$. When $f: E(G) \rightarrow\{1\}$ is the constant map, a $(\Gamma, f)$-coloring is a proper $|\Gamma|$-coloring. Therefore it is known that group coloring is a generalization of vertex coloring and furthermore that $\chi(G) \leq \chi_{g}(G)$.

Early research on group chromatic number and group choice number was almost entirely restricted to simple graphs, and usually considered only abelian groups in the definition of $\chi_{g}(G)$. To make a distinction here, we will define $\chi_{a}(G)$ to be the minimum $m$ such that $G$ is $A$-colorable for any abelian group $A$ with $|A| \geq m$. While it follows from the definitions that $\chi_{a}(G) \leq \chi_{g}(G)$ for

any given graph $G$, it has been remarked in [19] that the difference between these two values in not well-understood. Indeed, we are not aware of an example of a graph $G$ for which $\chi_{a}(G)<\chi_{g}(G)$. 
Recall that $\bar{\delta}(G)=\max \{\delta(H)$ : for any subgraph $H$ of $G\}$. The following property is shown in Lemma 4.2 of [23] (see also Corollary 2.3 of [26])

Theorem 1.2.1 For any multigraph $G, \chi_{g}(G) \leq \bar{\delta}(G)+1$.

This familiar bound will often be used throughout this dissertation, and will sometimes be referenced as the degeneracy bound. We also have the following analogues of Brooks' Theorem for group chromatic number that will often be used in the following chapters.

Theorem 1.2.2 Let $G$ be a graph.

(i) (Theorem 4.2 of [23]) If $G$ is simple and connected, then $\chi_{g}(G) \leq \Delta(G)+1$, where equality holds if and only if $G$ is a cycle or a complete graph.

(ii) (Theorem 1.3 of [26]) If $G$ is connected, then $\chi_{g}(G) \leq \Delta(G)+1$, where equality holds if and only if $G$ is a $k C_{n}$ or a $k K_{n}$, for some positive integers $k$ and $n$.

The following Lemma characterizes all graphs $G$ with $\chi_{g}(G)=2$, and will also be frequently used throughout this dissertation.

Lemma 1.2.3 (Theorem 3.1 of [23]) $\chi_{g}(G)=2$ if and only if $G$ is a forest.

The following Theorem is very useful in ruling out cases, and provides a property of group coloring that further generalizations do not share.

Theorem 1.2.4 (Theorem 2.2 of [23]) A graph $G$ is $\Gamma$-colorable if and only if each block of $G$ is $\Gamma$-colorable.

Corollary 1.2.5 $\chi_{g}(G)=\max \left\{\chi_{g}(B): B\right.$ is a block of $\left.G\right\}$

Group coloring, when first introduced in [14], was conceived as a dual to the concept of group connectivity, which is itself a generalization of nowhere-zero flows. While this thesis will not make use of this dual relationship, it would be incomplete without giving the involved definitions and properties. A more 
complete overview of group connectivity can be found in [19]. It is important to note that group connectivity is only defined for abelian groups, so the dual relationship only extends to $\chi_{a}$.

Let $A$ be an additive abelian group, let $G$ be a directed multigraph, and let $f \in F(G, A)$. The boundary of $f$ is a function $\partial f: V(G) \rightarrow A$ defined by:

$$
\partial f(v)=\sum_{e \in E^{+}(v)} f(e)-\sum_{e \in E^{-}(v)} f(e)
$$

A function $b: V(G) \rightarrow A$ is an $A$-valued zero sum function on $G$ if we have:

$$
\sum_{v \in V(G)} b(v)=0
$$

We denote the set of all such $A$-valued zero sum function on $G$ by $Z(G, A)$, and say that a graph $G$ is $A$-connected if $G$ has an orientation $D$ such that for every for every $b \in Z(D, A)$, there is some $f \in F\left(D, A-\left\{0_{A}\right\}\right)$ such that $b=\partial f$. This function $f$ is then called a nowhere-zero $(A, b)$-flow. Like group colorability, group connectivity is independent of orientation (see [14]). Finally, we define $\Lambda_{g}(G)=\min \{k \mid G$ is $A$-colorable for all $A$ with $|A| \geq k\}$, the group connectivity number of $G$. The dual relationship is as follows:

Theorem 1.2.6 [19] Let $G$ be a 2-connected graph and $A$ be an abelian group. Then $G$ is A-colorable if and only if $M^{*}(G)$ is A-connected.

Since we have only defined group connectivity for graphs and not matroids here, the above theorem will only work with planar $G$. When $G$ is planar, the $A$-connectivity of $M^{*}(G)$ is equivalent to the $A$-connectivity of $G^{*}$, the planar dual of $G$. As a direct consequence of Theorem 1.2.6 we have the following:

Corollary 1.2.7 Let $G$ be a 2-connected planar graph. Then $\chi_{a}(G)=\Lambda_{g}\left(G^{*}\right)$.

This Corollary 1.2.7 will be referenced in this dissertation to give context to some cited results, but will not be directly used in any proofs. 


\subsubsection{Group List Coloring}

Group coloring problems, defined in Subection 1.2.1, can be extended to a more general type of problem which will be called group list coloring in this dissertation. This generalization was introduced in [18] and can be defined as follows:

A list assignment of a graph $G$ is a map $L: V(G) \rightarrow 2^{\Gamma}$ that assigns to each vertex $v \in V(G)$ a list $L(v)$ of colors, and $L$ is a $k$-list if for any $v \in V(G)$, $|L(v)|=k$. Fix an orientation $D$ of $G$ and list assignment $L: V(G) \rightarrow 2^{\Gamma}$. For an $f \in F(G, \Gamma), G$ is $(\Gamma, L, f)$-colorable if there exists a map $c: V(G) \rightarrow$ $\cup_{v \in V(G)} L(v)$ such that $c(v) \in L(v)$, and $c(x)-c(y) \neq f(e)$ for any vertex $v \in V(G)$ and for arc $e=(x, y) \in E(G)$. If for any $f \in F(G, \Gamma), G$ has a $(\Gamma, L, f)$-coloring, then $G$ is $(\Gamma, L)$-colorable. We may note that this last property does not depend on the orientation $D$ of $G$.

If $G$ is $(\Gamma, L)$-colorable for any group $\Gamma$ of order at least $k$ and for any $k$-list assignment $L: V(G) \rightarrow 2^{\Gamma}$, then $G$ is $k$-group list colorable. The group list chromatic number of $G$, denoted $\chi_{g l}(G)$, is the minimum $k$ such that $G$ is $k$-group list colorable. It follows from the definitions that for a graph $G$, $\max \left\{\chi_{g}(G), \chi_{L}(G)\right\} \leq \chi_{g l}(G)$.

A list $L: V(G) \rightarrow 2^{\Gamma}$ is a $(\Gamma, D)$-list if for all $v \in V(G),|L(v)|=d(v)$. When the group $\Gamma$ is understood from the context, we often use a $D$-list in place of a $(\Gamma, D)$-list. A graph $G$ is $D$-group list colorable or $D$-group choosable if for any group $\Gamma$ with $|\Gamma| \geq \Delta(G)$ and for any $(\Gamma, D)$-list $L, G$ is $(\Gamma, L)$-colorable. It follows from the definitions that if a graph $G$ is $D$-group list colorable, then $\chi_{g l}(G) \leq \Delta(G)$.

Earlier research on group chromatic number and group choice number was almost entirely restricted to simple graphs, and usually considered only abelian groups in the definition of $\chi_{g}(G)$. Results in this dissertation will not require such restrictions. It is observed that, unlike classical coloring, $\chi_{g}(G)>\chi_{g}(\hat{G})$ is possible. For example, it follows from the results in Theorem 1.2.2 that $\chi_{g}\left(2 K_{2}\right)=$ 3 and $\chi_{g}\left(K_{2}\right)=2$, though surely $\chi\left(2 K_{2}\right)=\chi\left(K_{2}\right)=2$.

The investigation of $D$-choosabiliy plays an important role in the extension of 
Brooks' theorem to its group list coloring version in Chapter 3. The following two relevant lemmas were written and proved for simple graphs, but they also apply to multigraphs, with only superficial modification of their proofs. The proofs written here have been superficially adapted from the cited sources to include the case that $G$ might not be simple.

Lemma 1.2.8 (Chuang et al. Lemma 2.3 of [7]) Let $G$ be a multigraph, then $\chi_{g l}(G) \leq \bar{\delta}(G)+1$.

Proof. Define $t=\bar{\delta}(G)+1$, and define, for $1 \leq k \leq n, H_{k}=G\left[\left\{v_{1}, v_{2} \ldots v_{k}\right\}\right]$. Let $G$ be a multigraph, let $L$ be an $t$-list assignment, and let $f \in F(G, \Gamma)$ for some $\Gamma$ with $|\Gamma| \geq t$. Then by the definition of $\bar{\delta}(G)$ (see Section 1.1) we may order $V(G)=\left\{v_{1}, v_{2} \ldots v_{n}\right\}$ such that $d_{H_{k}}\left(v_{k}\right) \leq t-1$. We now construct a $(\Gamma, L, f)$ coloring of $G$ by induction: First choose an arbitrary color for $v_{1}$. Now note that since $d_{H_{k}}\left(v_{k}\right) \leq t-1$ we may extend a $\left(\Gamma,\left.L\right|_{V\left(H_{k-1}\right)},\left.f\right|_{E\left(H_{k-1}\right)}\right)$-coloring of $H_{k-1}$ to a $\left(\Gamma,\left.L\right|_{V\left(H_{k}\right)},\left.f\right|_{E\left(H_{k}\right)}\right)$-coloring of $H_{k}$ by simply choosing for $c\left(v_{k}\right)$ the element of $L\left(v_{k}\right)$ that is not forbidden by the previous color choices for $N_{H_{k}}\left(v_{k}\right)$.

Lemma 1.2.9 (Chuang et al. Lemma 2.7 of [7]) If a connected multigraph G has a connected induced D-group choosable subgraph $H$, then $G$ is D-group choosable.

Proof. Let $G$ be a multigraph, let $L$ be a $(\Gamma, D)$ list for some group $\Gamma$ with $|\Gamma| \geq \Delta(G)$, and let $f \in F(G, \Gamma)$. Assume that $H$ is a $D$-group choosable induced subgraph of $G$. We proceed by an induction on $|V(G)-V(H)|$ :

In the case that $|V(G)-V(H)|=0$, the result is holds trivially. Now assume that the result holds when $|V(G)-V(H)|=k$, and examine the case where $|V(G)-V(H)|=k+1$. Select $x \in V(G)-V(H)$ such that the distance between $H$ and $x$ is maximized. Then $G-x$ is connected and contains $H$ as an induced subgraph. Thus, by the inductive hypothesis, $G-x$ is $D$-group choosable. We may assume without loss of generality that all arcs incident to $x$ are directed away from $x$. Choose some arbitrary $t \in L(x)$ and define $\bar{L}: V(G)-x \rightarrow 2^{\Gamma}$ as follows: 


$$
\bar{L}(v)=\left\{\begin{array}{ll}
L(v)-\{-f(x v)+t\} & \text { if } v \in N_{G}(x) \\
L(v) & \text { otherwise }
\end{array}\right\}
$$

Since $G-x$ is $D$-group choosable, we may construct a $\left(\Gamma,\left.L\right|_{V(G)-x},\left.f\right|_{E(G-x)}\right)$ coloring $c$ of $G$. Considering the construction of $\bar{L}$, it is not difficult to see that we can extend $c$ to be a $(\Gamma, L, f)$-coloring of $G$ by setting $c(x)=t$.

\subsubsection{DP Coloring}

A further generalization of vertex coloring called DP-coloring or correspondence coloring has been recently introduced by Dvořák and Postle in [8]. We use a notation developed in [1].

Definition 1.2.10 Let $G$ be a multigraph. A cover of $G$ is a pair $(L, H)$, where $L$ is an assignment of pairwise disjoint sets to the vertices of $G$ and $H$ is a graph with vertex set $\bigcup_{v \in V(G)} L(v)$ satisfying the following:

1. $H[L(v)]$ is a complete graph for each $v \in V(G)$.

2. For each $u, v \in V(G), E_{H}[L(u), L(v)]$ is the union of $m_{G}(u, v)$ (possibly empty) matchings.

Now, if $(L, H)$ is a cover of a multigraph $G$. An $(L, H)$-coloring of $G$ is an independent set in $H$ of size $|V(G)|$, and graph $G$ with such a coloring is called $(L, H)$-colorable. A $k$-cover of a graph $G$ has $|L(v)|=k$ for each $v \in V(G)$. A $k$-cover $(L, H)$ of a simple graph $G$ is full if $E_{H}[L(u), L(v)]$ is a perfect matching for each $u v \in E(G)$. Similarly, a $k$-cover $(L, H)$ of a multigraph $G$ is full if $E_{H}[L(u), L(v)]$ is a union of $m_{G}(u, v)$ perfect matchings for each $u v \in E(G)$. If a graph $G$ is $(L, H)$-colorable for any $k$-cover $(L, H)$ then $G$ is DP- $k$-colorable. Similarly, we say that $G$ is DP-degree-colorable if it is $(L, H)$ colorable for any $L$ where $|L(v)|=d(v)$ for each $v \in V(G)$. The DP-chromatic number is defined as $\chi_{D P}(G)=\min \{k \in \mathbb{N}: G$ is DP- $k$-colorable $\}$. Unlike in group 
coloring and group list coloring, it is worth noting that DP coloring has the following property:

Proposition 1.2.11 If a multigraph $G$ is DP-k-colorable, then $G$ is $(L, H)$ colorable for any $(L, H)$ such that $|L(v)| \geq k$ for each $v \in V(G)$

Proof. Assume that $G$ is DP- $k$-colorable, and let $(L, H)$ be a cover of $G$ such that $|L(v)| \geq k$ for each $v \in V(G)$. We may arbitrarily remove vertices from $H$ until we form a $k$-cover $\left(L^{\prime}, H^{\prime}\right)$ of $G$. Since $G$ is DP- $k$-colorable, there is an $\left(L^{\prime}, H^{\prime}\right)$-coloring of $G$, i.e. an independent set $S$ in $H^{\prime}$ of size $|V(G)|$. Assume, by way of contradiction, that $S$ is not independent in $H$. Then there exist $x, y \in S$ with $x y \in E(H)$. Since $S \subseteq V\left(H^{\prime}\right) \subseteq V(H)$, and by construction of $H^{\prime}$, we then have $x y \in E\left(H^{\prime}\right)$ implying that $S$ is not independent in $H^{\prime}$, a contradiction. Therefore, $S$ is independent in $H$ as well, and thus it constitutes an $(L, H)$-coloring of $G$.

As with many other coloring notions, we have the following familiar degeneracy bound for $\chi_{D P}$ as well. It is not formally stated, but mentioned in passing by Dvořák and Postle in [8]. The proof does not differ meaningfully from that of its analogues Theorem 1.2.1 and Lemma 1.2.8 in group coloring and group list coloring, respectively.

Lemma 1.2.12 [8] $\chi_{D P}(G) \leq \bar{\delta}(G)+1$

Now consider the following Proposition:

Proposition 1.2.13 For any multigraph $G$ we have $\chi_{g l}(G) \leq \chi_{D P}(G)$.

Proof: Let $G$ be a multigraph with $\chi_{D P}(G) \leq k$. Let $\Gamma$ be a finite group, let $f \in F(G, \Gamma)$ and let $L: V(G) \rightarrow 2^{\Gamma}$ with $|\Gamma| \geq k$ and $|L(w)| \geq k$. Now to show that $\chi_{g l}(G) \leq k$ (and thus prove the proposition) it suffices to show that $G$ is $(\Gamma, f, L)$-colorable for such arbitrarily chosen $(\Gamma, f, L)$. We construct a $k$-cover $(K, H)$ as follows. For each $w \in V(G)$ label $K(w)$ with $L(w)$. For each $e \in E(G)$, 
define a matching $J_{e}$ on $K(u) \cup K(v)$ by $E\left(J_{e}\right):\{x y: x \in L(u), y \in L(v), x-y=$ $f(u v)\}$. Now let $E_{H}[L(u), L(v)]=\bigcup\left\{J_{e}: e \in[u v]\right\}$. Then since $\chi_{D P}(G) \leq k, G$ is $(K, H)$-colorable, so by the construction of $(K, H)$ we see that $G$ is $(\Gamma, f, L)$ colorable as well.

Together with previous bounds, we now have the following inequality for any multigraph $G$ :

$$
\chi(G) \leq \chi_{g}(G) \leq \chi_{g l}(G) \leq \chi_{D P}(G)
$$

Applying this inequality we may characterize all graphs $G$ with $\chi_{D P}(G)=2$

Lemma 1.2.14 $\chi_{D P}(G)=2$ if and only if $G$ is a nontrivial forest.

Proof: Assume $\chi_{D P}(G)=2$. Then $G$ is nontrivial and $\chi_{g}(G) \leq 2$ so that by Lemma 1.2.3 $G$ must be a forest. Assume that $G$ is a nontrivial forest. Then $\bar{\delta}(G)=1$ so that by Lemma 1.2.12 we must have $\chi_{D P}(G) \leq 2$. By Lemma 1.2.3 we have $\chi_{g}(G)=2$ and thus $\chi_{D P}(G)=2$ as well.

The following result by Bernshteyn, Kostochka, and Pron will be used in constructing counterexamples in Chapter 4. We may note that it generalizes 3.1.16.

Theorem 1.2.15 (Theorem 9 of [1]) If a multigraph $G$ is connected, then $G$ is not DP-degree-colorable if and only if each block of $G$ is one of the graphs $k K_{n}$ and $k C_{n}$ for some $n$ and $k$. 


\subsection{4 $S_{k}$ Coloring}

In [15], a generalization of DP-coloring is introduced which we will find useful. Let $S$ be a subset of $S_{k}$ for some integer $k>0$, where $S$ is closed under taking inverses. Let $\sigma: E(G) \rightarrow S$ such that $\sigma_{u v}=\left(\sigma_{v u}\right)^{-1}$ for each pair $u, v$ of adjacent vertices in $G$. We say that $G$ is $\sigma$-colorable if there exists a function $c: V(G) \rightarrow[k]$ such that $\sigma_{u v}(c(u)) \neq c(v)$ for any $u v \in E(G)$. We say that $G$ is $S$ - $k$-colorable if $G$ is $\sigma$-colorable for any $\sigma: E(G) \rightarrow S$ with the inverse value requirement as described above. We will demonstrate that, in some sense, this concept generalizes DP-coloring.

Proposition 1.2.16 A graph $G$ is $S_{k}$-k-colorable if and only if $\chi_{D P}(G) \leq k$.

Proof: First we assume that $G$ is $S_{k}$-k-colorable and let $(L, H)$ be a $k$-cover of $G$. We may assume without loss of generality that $(L, H)$ is full, otherwise we might consider a more "difficult" $H$ with the desired property. For each $v \in V(G)$, we may arbitrarily identify $L(v)$ with $[k]$. Now for each $u v \in E(G)$ we may covert $H[L(u), L(v)]$ into a permutation $\sigma_{u v}$ where $\sigma_{u v}(i)=j$ when $i j \in E_{H}[L(u), L(v)]$. If $c$ is a $\sigma$-coloring of $G$, then it is also an $(L, H)$-coloring of $G$. Since we assumed that $G$ is $S_{k^{-}} k$-colorable, and $(L, H)$ was arbitrarily chosen, we have $\chi_{D P}(G) \leq k$.

Conversely, we assume that $\chi_{D P}(G) \leq k$. Let $\sigma: E(G) \rightarrow S_{k}$ be such that $\sigma_{u v}=\left(\sigma_{v u}\right)^{-1}$ for each pair $u, v$ of adjacent vertices in $G$. We convert $\sigma$ to a cover $(L, H)$ of $G$ as follows: For each $v \in V(G)$ let $L(v)=[k]$. For each $u v \in E(G)$ let $E_{H}[L(u), L(v)]=\left\{x y: x \in L(u), y \in L(v)\right.$, and $\left.\sigma_{u v}(x)=y\right\}$. Finally, let $H[L(v)]=K_{k}$ for each $v \in V(G)$. No other edges are present in $H$, besides those described above. Now as $c$ is an $(L, H)$-coloring of $G$, then it is also a $\sigma$-coloring of $G$. Since $\chi_{D P}(G) \leq k, G$ is $(L, H)$-colorable for any $k$-cover $(L, H)$ of $G$. Therefore $G$ is $\sigma$-colorable for any $\sigma$ as described, so by definition $G$ is $S_{k^{-}} k$-colorable.

This notion of $S_{k}$-coloring will not be extensively explored here, but it will be applied to several DP coloring problems in Chapter 4. 


\section{Chapter 2}

\section{Group Coloring}

In this chapter we first describe previous results in group coloring, and then present new ones. See Subsection 1.2.1 for definitions, basic properties, and a more explicit statement of certain previously published results.

\subsection{Previous Results and Immediate Consequences}

Group coloring of a graph was introduced by Jaeger et al. in [14] for abelian group as the dual concept of group-connectivity of graphs. The main substantive coloring result in this paper, besides the conception of group coloring, was the bound $\chi_{g}(G) \leq 6$ for simple planar $G$. This result was improved to $\chi_{g}(G) \leq 5$ in [24], and the result was at the same time generalized to $K_{5}$-minor-free graphs. It is shown in [22] that $\chi_{g}(G) \leq 5$ for $K_{3,3}$-minor-free graphs as well. All of these bound have been proven to be sharp by an example presented in [17] of a planar graph $G$ with $\chi_{g}(G)=5$. It is worth noting that this last result shows that Hadwiger's Conjecture ([10]) does not extend to group colorings.

For higher genus, it is not hard to see that the degree conditions used to prove the Heawood Bound (see [11]) for chromatic number will also suffice to show that the same bound bound applies for graphs embeddable in surfaces other than the sphere. Later authors noted that the same reasoning could be extended to $\chi(G)$ on non-orientable surfaces, and again these results apply to group coloring as 
well:

Theorem 2.1.1 For a graph $G$ embedded in a surface $S$ of Euler Characteristic $e(S) \neq 2$, we have $\chi_{g}(G) \leq\left\lfloor\frac{7+\sqrt{49-24 e(S)}}{2}\right\rfloor$.

It is now natural to ask now whether the bound in Theorem 2.1.1 is tight. Franklin found in [9] that Heawood's Bound is not tight on the Klein sack. His

proof can be generalized to show that $\chi_{g}(G) \leq 6$ for $G$ embedded on the Klein Bottle as well. However, Ringel and Youngs have finished showing in [28] that the Heawood bound is tight for all orientable surfaces other than the sphere, and their proof can be easily generalized to $\chi_{g}$ : since $\chi(G) \leq \chi_{g}(G)$, we see that the bound in Theorem 2.1.1 is tight for group chromatic number as well, as far as orientable surfaces are concerned.

Moving away from embedding problems, we note that Lai and Zhang [23] proved analogues of Brooks' Theorem [4] and the Nordhaus-Gaddum Theorem [27], as well as giving examples of bipartite graphs $G$ with arbitrarily high $\chi_{g}(G)$. Shortly thereafter Král [16] showed that group coloring is $\Pi_{2}^{P}$-Complete. Then Lai, Li, and $\mathrm{Yu}$ [20] extended the work of Szekeres and Wilf [30], a generalization of Brooks' Theorem, to group coloring. Next, Lai and Li extended the Brooks Theorem analogue from [23] to the multigraph case in [26]. Most recently, Li has determined the group chromatic number of all Halin Graphs in [25].

\subsection{Group Coloring and Group Structure}

\subsubsection{Motivation}

In [14] a problem is posed: for any two abelian groups $\Gamma_{1}$ and $\Gamma_{2}$ with $\left|\Gamma_{1}\right|=\left|\Gamma_{2}\right|$, is a $\Gamma_{1}$-connected graph also $\Gamma_{2}$-connected? In [13], R. Hǔsek, L. Mohelníková, R. Šámal first answer this question with the following conclusion.

Theorem 2.2.1 (R. Hǔsek, L. Mohelniková, R. Šámal, Theorem 3 of [13]) Let $\mathbb{Z}_{n}$ denote the cyclic group of order $n$ and $\mathbb{Z}_{n}^{2}=\mathbb{Z}_{n} \times \mathbb{Z}_{n}$. Each of the following 
holds.

(i) There exists a graph that is $\mathbb{Z}_{4}$-connected but not $\mathbb{Z}_{2}^{2}$-connected.

(ii) There exists a graph that is $\mathbb{Z}_{2}^{2}$-connected but not $\mathbb{Z}_{4}$-connected.

Naturally there is a corresponding problem in group coloring: for given groups $\Gamma_{1}$ and $\Gamma_{2}$ with $\left|\Gamma_{1}\right|=\left|\Gamma_{2}\right|$, is a $\Gamma_{1}$-colorable graph also $\Gamma_{2}$-colorable? Equivalently, we may ask whether any aspect of group structure (other than the cardinality of the group) plays a role in group colorings. The purpose of this research is an attempt to address this question. More generally, we are to investigate answers to the following question.

Question 2.2.2 Is the $\Gamma$-colorability of a graph $G$ only dependent on $G$ and $|\Gamma|$ ?

As the graphs showing the validity of Theorem 2.2.1 in [13] are nonplanar, we cannot apply duality and quote Theorem 2.2.1 to answer Question 2.2.2. In this paper, we show that there are infinitely many ordered triples $\left(G, \Gamma_{1}, \Gamma_{2}\right)$ in which $G$ is a graph, $\Gamma_{1}$ and $\Gamma_{2}$ are groups with $\left|\Gamma_{1}\right|=\left|\Gamma_{2}\right|$, and $G$ is $\Gamma_{1}$-colorable but not $\Gamma_{2}$-colorable. While the justification of Theorem 2.2.1 uses computer checking, we develop a concept called snarls which characterize certain phenomena observed in group colorings, and use snarls to construct our examples. In the following subsections we will develop the concept of snarls, construct examples negating Question 2.2.2, and finally outline possible refinements of Question 2.2.2.

\subsubsection{The Snarl}

To give a negative answer to Question 2.2.2, it is useful to first introduce the concept of a snarl. This section will define this phenomenon as well as give sufficient and necessary conditions for a snarl to occur. Throughout this section, $G$ denotes a graph, $\Gamma$ denotes a multiplicative group with identity 1 , and as in [12], for a subset $F \subseteq \Gamma$, define $F^{-1}=\left\{x^{-1}: x \in F\right\}$. Before we consider the definition of a snarl, it will be useful to prove a small proposition that will remove some cases from consideration and significantly simplify the notation. 
Definition 2.2.3 Let $G$ be a multigraph under an orientation $D$ such that any two parallel edges have the same direction, and let $\Gamma$ be a group such that $|u v| \leq$ $|\Gamma|$ for each $u v \in E(\hat{G})$. If $f \in F(G, \Gamma)$ such that $\left.f\right|_{[u v]}$ is injective for each $u v \in E(\hat{G})$, then we say that $f$ is piecewise injective.

Proposition 2.2.4 Let $G$ be a multigraph and let $\Gamma$ be a group such that $|u v| \leq$ $|\Gamma|$ for each uv $\in \hat{E(G)}$. If $G$ is $(\Gamma, f)$-colorable for every piecewise injective $f \in F(G, \Gamma)$, then $G$ is $\Gamma$-colorable.

Proof: Let $G$ and $\Gamma$ be as described above and fix some orientation $D$ of $G$ such that any two parallel edges have the same direction. Assume that $G$ is $(\Gamma, f)$-colorable for any $f \in F(G, \Gamma)$ such that $f$ is piecewise injective. Let $f_{0} \in F(G, \Gamma)$ such that $\left.f_{0}\right|_{[u v]}$ is not piecewise injective. We now recursively define, for some $p \in \mathbb{N}, f_{1}, f_{2} \ldots f_{p}$ as follows: Choose $[u v]$ such that $u v \in E(\hat{G})$ and any $e_{1}, e_{2} \in[u v]$ with $e_{1} \neq e_{2}$ such that $f_{i}\left(e_{1}\right)=f_{i}\left(e_{2}\right)$. Let $f_{i+1}\left(e_{1}\right)=f_{i}\left(e_{1}\right)$ and $f_{i+1}\left(e_{2}\right) \in \Gamma-\left\{f_{i}(e): e \in[u v]\right\}$. Since we consider only finite graphs, this recursion will eventually halt, yielding $f_{p} \in F(G, \Gamma)$ such that $\left.f_{p}\right|_{[u v]}$ is piecewise injective. By assumption, there exists a $\left(\Gamma, f_{p}\right)$-coloring $c$ of $G$ under $D$. Since $f_{0}([u v]) \subseteq f_{p}([u v])$ for each $u v \in E(\hat{G}), c$ must also be a $\left(\Gamma, f_{0}\right)$-coloring of $G$ under $D$. Since $f_{0}$ was arbitrarily chosen, it follows directly from the definition that $G$ is $\Gamma$-colorable.

With Proposition 2.2.4 in mind, for the remainder of the paper we need only consider piecewise injective $f \in F(G, \Gamma)$. While doing so, we will work under the assumption that $G$ is under an orientation $D$ such as is described in Definition 2.2.3.

Definition 2.2.5 For a fixed piecewise injective $f \in F(G, \Gamma)$ and an edge uv $\in$ $E(G)$, let $f\left(A_{u v}\right)=\left\{f(e): e \in A_{u v}\right\}$ where $A_{u v} \subseteq[u v] . A(u, v ; f)$-snarl is a 3-tuple $\left(L_{u}, L_{v}, A_{u v}\right)$ with $\left|L_{u}\right|=\left|L_{v}\right|=\left|A_{u v}\right|$ and $L_{u}, L_{v} \subseteq \Gamma$, satisfying the property that for any $a \in L_{u}, f\left(A_{u v}\right)^{-1} a=L_{v}$. 
To simplify our notation, when $f$ is understood or assumed in the context, we often use a $(u, v)$-snarl for a $(u, v ; f)$-snarl. We further define that a $(u, v)$-snarl with $A_{u v}=[u v]$ is a full width $(u, v)$-snarl.

Proposition 2.2.6 For a given piecewise injective $f \in F(G, \Gamma)$, if there exists a $(u, v)$-snarl $\left(L_{u}, L_{v}, A_{u v}\right)$, then each of the following holds.

(i) For any $e_{p} \in A_{u v}$ and $a_{q} \in L_{u}$, there exists $b_{z} \in L_{v}$ such that $f\left(e_{p}\right)^{-1} a_{q}=b_{z}$.

(ii) For any $a_{p} \in L_{u}$ and $b_{q} \in L_{v}$, there exists $e_{z} \in A_{u v}$ such that $f\left(e_{z}\right)^{-1} a_{p}=b_{q}$.

(iii) For any $e_{p} \in A_{u v}$ and $b_{q} \in L_{v}$, there exists $a_{z} \in L_{u}$ such that $f\left(e_{p}\right)^{-1} a_{z}=b_{q}$. (iv) For any $a_{0} \in \Gamma-L_{u}$, we have $\left\{f(e)^{-1} a_{0}: e \in A_{u v}\right\} \cap L_{v}=\varnothing$.

Proof. By Definition 2.2.5, (i) and (ii) follow directly. We argue by contradiction to prove (iii) and assume that for any $a_{z} \in L_{u}, f\left(e_{p}\right)^{-1} a_{z} \neq b_{q}$. Then $f\left(e_{p}\right)^{-1} L_{u} \subsetneq L_{v}$. This implies that there exist distinct $a_{r}$ and $a_{s}$ in $L_{u}$ and $b_{t} \in L_{v}$ such that $f\left(e_{p}\right)^{-1} a_{r}=f\left(e_{p}\right)^{-1} a_{s}=b_{t}$, which forces $a_{r}=a_{s}$, a contradiction. Hence (iii) must hold.

We also argue by contradiction to prove (iv) and assume that there exists an $a_{0} \in \Gamma-L_{u}$ and an $e_{p} \in A_{u v}$ such that $f\left(e_{p}\right)^{-1} a_{0}=b_{q}$ for some $b_{q} \in L_{v}$. Then, by (iii), there must be an $a_{z} \in L_{u}$ such that $f\left(e_{p}\right)^{-1} a_{z}=b_{q}$, forcing $a_{0}=a_{z} \in L_{u}$, a contradiction.

We will now work towards necessary and sufficient conditions for a snarl to occur. Through the rest of this subsection, we assume that $G$ is a given graph, that $u v \in E(G)$, and unless otherwise stated, we always use $\left(L_{u}, L_{v}, A_{u v}\right)$ to denote a $(u, v)$-snarl, assuming the existence of it.

Definition 2.2.7 For such a $(u, v)$-snarl, $\left(L_{u}, L_{v}, A_{u v}\right)$, we may define:

$$
H_{1}=\left\{a_{i} a_{j}^{-1}: a_{i}, a_{j} \in L_{u}\right\}, H_{2}=\left\{a_{i}^{-1} a_{j}: a_{i}, a_{j} \in L_{u}\right\} \text {, and } H_{3}=\left\{b_{i} b_{j}^{-1}:\right.
$$
$\left.b_{i}, b_{j} \in L_{v}\right\}$.

Proposition 2.2.8 For a given piecewise injective $f \in F(G, \Gamma)$, and $a(u, v)$ $\operatorname{snarl}\left(L_{u}, L_{v}, A_{u v}\right)$, each of the following holds.

(i) If $F_{1}=\left\{f_{i} f_{j}^{-1}: f_{i}, f_{j} \in f\left(A_{u v}\right)\right\}$, then $H_{1}=F_{1}$. 
(ii) If $F_{2}=\left\{b_{i}^{-1} b_{j}: b_{i}, b_{j} \in L_{v}\right\}$, then $H_{2}=F_{2}$.

(iii) If $F_{3}=\left\{f_{i}^{-1} f_{j}: f_{i}, f_{j} \in f\left(A_{u v}\right)\right\}$, then $H_{3}=F_{3}$.

(iv) Given any $a_{i}, a_{j}, a_{k} \in L_{u}$, there exists $a_{l} \in L_{u}$ such that $a_{i} a_{j}^{-1}=a_{l} a_{k}^{-1}$.

(v) Given any $b_{i}, b_{j}, b_{k} \in L_{v}$, there exists $b_{l} \in L_{v}$ such that $b_{i} b_{j}^{-1}=b_{l} b_{k}^{-1}$.

Proof. For any $a_{i}, a_{j} \in L_{u}$, by Proposition 2.2.6(ii), there exist $f_{y}, f_{z} \in f\left(A_{u v}\right)$ and $b_{q} \in L_{v}$ such that $a_{i} a_{j}^{-1}=f_{y} b_{q}^{-1}\left(f_{z} b_{q}^{-1}\right)^{-1}=f_{y} f_{z}^{-1} \in F_{1}$, implying $H_{1} \subseteq F_{1}$. Conversely, for any $f_{i}, f_{j} \in f\left(A_{u v}\right)$, by Proposition 2.2.6(iii) there exist $a_{i}, a_{j} \in L_{u}$ and $b_{q} \in L_{v}$ such that $f_{i} f_{j}^{-1}=a_{i} b_{q}\left(a_{j} b_{q}^{-1}\right)^{-1}=a_{i} a_{j}^{-1} \in H_{1}$, implying $F_{1} \subseteq H_{1}$. This proves (i). The proofs for (ii) and (iii) are similar and so are omitted.

By Proposition 2.2.6(i), there exists an element $b_{x} \in L_{v}$ such that $f_{z}=a_{k} b_{x}^{-1}$, and by Proposition 2.2.6(iii), there exists $a_{l} \in L_{u}$ such that $f_{y}=a_{l} b_{x}^{-1}$. Therefore, $a_{i} a_{j}^{-1}=f_{y} f_{z}^{-1}=\left(a_{l} b_{x}^{-1}\right)\left(a_{k} b_{x}^{-1}\right)^{-1}=a_{l} a_{k}^{-1}$. This proves (iv).

Finally, by Proposition 2.2.6(iii) there exists $a_{z} \in L_{u}$ such that $f_{x}^{-1} a_{z}=b_{k}$ and by Proposition 2.2.6(i) there exists $b_{l} \in L_{v}$ such that $f_{w}^{-1} a_{z}=b_{l}$. Thus $b_{i} b_{j}^{-1}=f_{w}^{-1} f_{x}=\left(a_{z} b_{l}^{-1}\right)^{-1}\left(a_{z} b_{k}^{-1}\right)=b_{l} a_{z}^{-1} a_{z} b_{k}^{-1}=b_{l} b_{k}^{-1}$. This proves (v).

There is some symmetry at play here, so there are more properties similar to those written in Proposition 2.2.8, including an " $f$ " property corresponding to (iv) and (v). In the interest of brevity, Proposition 2.2.8 is limited to include only what is needed to prove Theorem 2.2.10.

Lemma 2.2.9 The subsets $H_{1}, H_{2}, H_{3}$ defined in Definition 2.2.7 are conjugate subgroups of $\Gamma$.

Proof. Given $h_{1}, h_{2} \in H_{1}$, by Definition 2.2.7, there exist some $a_{i}, a_{j}, a_{p}, a_{q} \in L_{u}$ such that we have $h_{1}=a_{i} a_{j}^{-1}$ and $h_{2}=a_{p} a_{q}^{-1}$. By Proposition 2.2.8(iv), we may also write $h_{1}=a_{l} a_{q}^{-1}$ for some element $a_{l}$ so that $h_{1} h_{2}^{-1}=a_{l} a_{q}^{-1}\left(a_{p} a_{q}^{-1}\right)^{-1}=$ $a_{l} a_{p}^{-1} \in H_{1}$. As $H_{1} \neq \varnothing, H_{1}$ is a subgroup of $\Gamma$. To prove the lemma, we first justify the following two claims.

Claim 1: For any element $a \in L_{u}, a H_{2}=L_{u}=H_{1} a$.

By Definition 2.2.7, we have $H_{2}=\left\{a_{i}^{-1} a_{j}: a_{i}, a_{j} \in L_{u}\right\}$. Thus for any $a_{j} \in L_{u}$, as $a \in L_{u}$, we have $a_{j}=a a^{-1} a_{j} \in a H_{2}$, and so $L_{u} \subseteq a H_{2}$. Conversely, 
take an arbitrary element $a a_{i}^{-1} a_{j} \in a H_{2}$. By Proposition 2.2.8(iv), there exists an element $a_{l} \in L_{u}$, with which we may write $a a_{i}^{-1} a_{j}=a_{l} a_{j}^{-1} a_{j}=a_{l} \in L_{u}$. This implies that $a H_{2} \subseteq L_{u}$, and so $a H_{2}=L_{u}$. Again by Definition 2.2.7, we have $H_{1}=\left\{a_{i} a_{j}^{-1}: a_{i}, a_{j} \in L_{u}\right\}$. Thus for any $a_{i} \in L_{u}$, as $a \in L_{u}$, we have $a_{i}=a_{i} a^{-1} a \in H_{1} a$ as well, and so $L_{u} \subseteq H_{1} a$. Conversely, pick an arbitrary element $a_{i} a_{j}^{-1} a \in H_{1} a$. By Proposition 2.2.8(iv), there exists an element $a_{l} \in L_{u}$ so that $a_{i} a_{j}^{-1} a=a_{l} a^{-1} a=a_{l} \in L_{u}$. Thus $H_{1} a \subseteq L_{u}$, and so $H_{1} a=L_{u}$. This completes the proof of the claim.

Claim 2: For any element $b \in L_{v}, b H_{2}=L_{v}=H_{3} b$.

By Proposition 2.2.8(ii), we have $H_{2}=\left\{b_{i}^{-1} b_{j}: b_{i}, b_{j} \in L_{v}\right\}$. Thus for any $b_{j} \in L_{v}$, as $b \in L_{v}$, we have $b_{j}=b b^{-1} b_{j} \in b H_{2}$, and so $L_{v} \subseteq b H_{2}$. Conversely, take an arbitrary element $b b_{i}^{-1} b_{j} \in b H_{2}$. By Proposition 2.2.8(v), there exists an element $b_{l} \in L_{v}$ such that $b b_{i}^{-1} b_{j}=b_{l} b_{j}^{-1} b_{j}=b_{l} \in L_{v}$. This proves $b H_{2}=L_{v}$. By Definition 2.2.7, we have $H_{3}=\left\{b_{i} b_{j}^{-1}: b_{i}, b_{j} \in L_{v}\right\}$. Thus, for any $b_{i} \in L_{v}$, as $b \in L_{v}$, we have $b_{i}=b_{i} b^{-1} b \in H_{3} b$, and so $L_{v} \subseteq H_{3} b$. Conversely, for any $b_{i} b_{j}^{-1} b \in H_{3} b$, by Proposition 2.2.8(v), there exists an element $b_{l} \in L_{v}$ such that $b_{i} b_{j}^{-1} b=b_{l} b^{-1} b=b_{l} \in L_{v}$, and so $H_{3} b \subseteq L_{v}$. This validates the claim.

By Claims 1 and 2, for elements $a \in L_{u}$ and $b \in L_{v}$, we have $H_{1}=a H_{2} a^{-1}$ and $H_{3}=b H_{2} b^{-1}$. Thus $H_{2}$ and $H_{3}$ are conjugates of $H_{1}$, and so $H_{1}, H_{2}$ and $H_{3}$ are conjugates of one another. It is well-known (see [12] Chapter II Section 4) that the conjugates of a subgroup is itself a subgroup. Thus $H_{1}, H_{2}, H_{3}$ are conjugate subgroups of $\Gamma$.

Now we may show some sufficient and necessary conditions for a snarl to occur:

Theorem 2.2.10 Let $f \in F(G, \Gamma)$ be a given piecewise injective mapping. For some $u v \in E(G)$, let $L_{u}, L_{v}, F \subseteq \Gamma$ such that for some $A_{u v} \subseteq[u v]$, we have $F=f\left(A_{u v}\right)$. Then the following are equivalent.

(i) $\left(L_{u}, L_{v}, A_{u v}\right)$ is a $(u, v)$-snarl.

(ii) There exist conjugate subgroups $H_{1}, H_{2}$, and $H_{3}$ of $\Gamma$ and elements $a, b, f \in$ $\Gamma$ satisfying $a b^{-1}=f$ such that $L_{u}=H_{1} a=a H_{2}, L_{v}=H_{3} b=b H_{2}$, and 
$F=H_{1} f=f H_{3}$.

Proof. By Lemma 2.2.9, (i) implies (ii). Assume that (ii) holds. We are to show that Definition 2.2.5 must hold. For any $a_{j} \in L_{u}$, there exists an element $h_{1} \in H_{1}$ such that $a_{j}=h_{1} a$, and so $\left\{f(e)^{-1} a_{j}: e \in A_{u v}\right\}=F^{-1} a_{j}=\left(H_{1} f\right)^{-1} h_{1} a$. As

$h_{1} \in H_{1}$ and as $H_{1}$ is a subgroup, $H_{1}^{-1} h_{1}=H_{1}^{-1}$, and so we have $\left(H_{1} f\right)^{-1} h_{1} a=$ $f^{-1} H_{1}^{-1} h_{1} a=f^{-1} H_{1}^{-1} a=\left(H_{1} f\right)^{-1} a=\left(f H_{3}\right)^{-1} a=H_{3}^{-1} f^{-1} a=H_{3} b=L_{v}$. It follows, by Definition 2.2.5, $\left(L_{u}, L_{v}, A_{u v}\right)$ is a $(u, v)$-snarl.

Corollary 2.2.11 follows from Theorem 2.2.10 immediately:

Corollary 2.2.11 Let $G$ be a graph, let $A$ be an abelian group, and $f \in F(G, A)$ be a fixed piecewise injective mapping. For some uv $\in E(G)$, let $L_{u}, L_{v}, F \subseteq A$ be subsets of $A$ such that for some $A_{u v} \subseteq[u v]$, we have $F=f\left(A_{u v}\right)$. Then the following are equivalent.

(i) $\left(L_{u}, L_{v}, A_{u v}\right)$ is a $(u, v)$-snarl.

(ii) There exists a subgroup $H$ of $A$ and elements $a, b, f \in A$ satisfying $a b^{-1}=f$ such that $L_{u}=a H, L_{v}=b H$, and $F=f H$.

Proof. By Theorem 2.2.10, Corollary 2.2.11(i) holds if and only if that there exist conjugate subgroups $H_{1}, H_{2}$, and $H_{3}$ of $A$ and elements $a, b, f \in A$ satisfying $a b^{-1}=f$ such that $L_{u}=H_{1} a=a H_{2}, L_{v}=H_{3} b=b H_{2}$, and $F=H_{1} f=f H_{3}$. Since $A$ is abelian, and since $H_{1}, H_{2}$, and $H_{3}$ are conjugate subgroups of $A$, we have $H:=H_{1}=H_{2}=H_{3}$, and so in this case, Theorem 2.2.10(ii) holds if and only if Corollary 2.2.11(ii) holds.

\subsubsection{Counterexamples}

We now use the characterization of a snarl developed in the previous subsection to give a counterexample for Question 2.2.2 in the two propositions that follow. We denote by $A_{4}$ the alternating group of degree 4 . Recall that $\left|A_{4}\right|=12$.

Proposition 2.2.12 The graph $6 K_{3}$ is $A_{4}$-colorable. 
Proof: Let $f \in F\left(6 K_{3}, A_{4}\right)$, and let $v_{1}, v_{2}$, and $v_{3}$ denote the three vertices of $6 K_{3}$. To show that $6 K_{3}$ is $A_{4}$-colorable, it suffices to construct an $\left(A_{4}, f\right)$-coloring of $6 K_{3}$. First, we arbitrarily choose $c\left(v_{1}\right) \in A_{4}$. With Proposition 2.2.4 in mind, we may assume that $f$ is piecewise injective. This leaves us with at exactly 6 choices for $c\left(v_{2}\right)$ (which we denote by $L_{2}$ ) and exactly 6 choices for $c\left(v_{3}\right)$ (which we denote by $L_{3}$ ). We may also assume without loss of generality that each arc in $\left[v_{2} v_{3}\right]$ is oriented towards $v_{3}$.

Assume by way of contradiction that for each $\gamma \in L_{2}$ we have $\left(f\left[v_{2} v_{3}\right]\right)^{-1} \gamma=$ $L_{3}$. Then we have, by Definition 2.2.5, a full-width $v_{2} v_{3}$-snarl. By Theorem 2.2.10 $A_{4}$ must then have a subgroup $H$ with $|H|=6$, though it is well known that $A_{4}$ has no subgroup of order 6 . We assume then that there exists $\gamma \in L_{2}$ such that $L_{3} \not \subset\left(f\left[v_{2} v_{3}\right]\right)^{-1} \gamma$, and so we may set $c\left(v_{2}\right)=\gamma$ and $c\left(v_{3}\right) \in\left(f\left[v_{2} v_{3}\right]\right)^{-1} \gamma-L_{3}$ to complete the coloring.

Proposition 2.2.13 The graph $6 K_{3}$ is not $\mathbb{Z}_{12}$-colorable.

Proof: Define $\Gamma_{e}=2 \mathbb{Z}_{12}$ to be the cyclic subgroup of $\mathbb{Z}_{12}$ with 6 elements, and let $\Gamma_{o}=\mathbb{Z}_{12}-\Gamma_{e}$. Let $v_{1}, v_{2}$, and $v_{3}$ denote the three vertices of $6 K_{3}$. We may assume without loss of generality that arcs are oriented from $v_{1}$ to $v_{2}, v_{2}$ to $v_{3}$, and $v_{3}$ to $v_{1}$. We define $f \in F\left(G, \mathbb{Z}_{12}\right)$ with $f\left[v_{1} v_{2}\right]=f\left[v_{2} v_{3}\right]=f\left[v_{3} v_{1}\right]=\Gamma_{e}$. To complete the proof, it suffices to show that $6 K_{3}$ is not $\left(\mathbb{Z}_{12}, f\right)$-colorable. Indeed, if we choose $c\left(v_{1}\right) \in \Gamma_{o}$, then by the definition of $f$, we must have $c\left(v_{2}\right) \in \Gamma_{e}$ (yielding a conflict at $\left[v_{3} v_{1}\right]$ ) so we cannot choose $c\left(v_{1}\right) \in \Gamma_{o}$. A similar argument shows that $c\left(v_{1}\right) \in \Gamma_{e}$ cannot be chosen either, and thus there can be no $\left(\mathbb{Z}_{12}, f\right)$ coloring of $6 K_{3}$.

Later, we shall show that for any abelian group $\Gamma$ with $|\Gamma|=12,6 K_{3}$ is not $\Gamma$-colorable. The important structural distinction here lies in the fact that $A_{4}$ has no subgroup of order 6 , whereas $\mathbb{Z}_{12}$ does. Since $\left|A_{4}\right|=\left|\mathbb{Z}_{12}\right|$, it is clear that group structure does play some role in group coloring, and we can answer Question 2.2.2 in the negative.

Before constructing more counterexamples it will be useful to define some terminology. First, consider the following well-known theorem of Lagrange: 
Theorem 2.2.14 If $H$ is a subgroup of a finite group $\Gamma$, then $|H|$ divides $|\Gamma|$.

It is important to note that the converse of Theorem 2.2.14 does not always hold; there exist many groups $\Gamma$ such that $h$ divides $|\Gamma|$ but $\Gamma$ has no subgroup of order $h$. Furthermore, this failure of the converse was vital to constructing the counter-example for Question 2.2.2 in Propositions 2.2.12 and 2.2.13. The converse of Theorem 2.2.14 has in fact been studied widely, but there is no known characterization of all groups for which it holds.

Definition 2.2.15 A group $\Gamma$ is called a CLT group or a Lagrangian group if for each $h$ such that $h$ divides $|\Gamma|$ there is a subgroup $H$ of $\Gamma$ with $|H|=h$.

It is well known (see [12] II Corollary 2.4) that any abelian group is a CLT group. Therefore further counterexamples for Question 2.2.2 using the same technique as above must have at least one of $\Gamma_{1}$ or $\Gamma_{2}$ be non-abelian. Any such counterexample must specifically include a group that is not CLT. The following lemma appears as an exercise in [29] and gives an infinite set of such non-CLT groups:

Lemma 2.2.16 For $n \geq 5$, the symmetric group $S_{n}$ has no subgroup $H$ with $2<\left[S_{n}: H\right]<n$.

The proof is heavy on algebra, so it is given in Appendix 5.1. We shall use Lemma 2.2.16 to construct an infinite set of counterexamples for Question 2.2.2.

Theorem 2.2.17 For each $n \geq 5$ and $2<t<n, \frac{n !}{t} K_{t+1}$ is $S_{n}$-colorable.

Proof: Let $G=\frac{n !}{t} K_{t+1}$ and let $f \in F\left(G, S_{n}\right)$ be piecewise injective. Fix two vertices $u, v \in V(G)$ and define $J=G-[u v]$ to be the graph formed by removing all parallel edges in $[u v]$. Then $\bar{\delta}(J)=(t-1) \frac{n !}{t}<n !=\left|S_{n}\right|$. By Theorem 1.2.1, $\left|S_{n}\right| \geq \bar{\delta}(J)+1 \geq \chi_{g}(J)$ and so $J$ is $S_{n}$-colorable. In particular, $J$ has an $\left(S_{n}, f \mid J\right)$-coloring $c$. We will show that at least one such $\left(S_{n}, f \mid J\right)$-coloring of $J$ is also an $\left(S_{n}, f\right)$-coloring of $G$. Since $d_{J}(u)=d_{J}(v)=(t-1) \frac{n !}{t}<\left|S_{n}\right|$, we may modify $c$ by changing $c(u)$ and $c(v)$, and it will still be an $\left(S_{n}, f \mid J\right)$-coloring of $J$. To be precise, we have at least $\left|S_{n}\right|-d_{J}(u)=\frac{n !}{t}$ choices for modifying $c(u)$ and 
likewise at least $\frac{n !}{t}$ choices for modifying $c(v)$. Let $L_{u}, L_{v} \subset S_{n}$ denote the sets of choices for $c(u)$ and $c(v)$ respectively. We may assume without loss of generality that each arc in $[u v]$ is oriented towards $v$.

First we consider the case where $\left|L_{u}\right|>\frac{n !}{t}$ or $\left|L_{v}\right|>\frac{n !}{t}$. Without loss of generality we assume $\left|L_{u}\right|>\frac{n !}{t}$. Then we may arbitrarily choose $c(v) \in L_{v}$, and be assured that at least one choice remains for $c(u) \in u$.

Now we consider the case where $\left|L_{u}\right|=\left|L_{v}\right|=\frac{n !}{t}$. Assume by way of contradiction that for each $\gamma \in L_{u}$ we have $(f[u v])^{-1} \gamma=L_{v}$. Then we have, by Definition 2.2.5, a full-width $u v$-snarl. By Theorem 2.2.10 $S_{n}$ must then have a subgroup $H$ with $|H|=|u v|=\frac{n !}{t}$. However, this would imply that $\left[S_{n}: H\right]=t$, contradicting Lemma 2.2.16. We assume then that there exists $\gamma \in L_{u}$ such that $L_{v} \not \subset(f[u v])^{-1} \gamma$, and so we may set $c(u)=\gamma$ and $c(v) \in(f[u v])^{-1} \gamma-L_{v}$. Now $c$ is an $\left(S_{n}, f\right)$-coloring of $G$, and thus $G$ is $S_{n}$-colorable.

Theorem 2.2.18 Let $\Gamma$ be a group with $|\Gamma|=$ st and let $H$ be a subgroup of $\Gamma$ such that $|H|=t$. Then $t K_{s+1}$ is not $\Gamma$-colorable.

Proof: Let $\Gamma$ be a group with $|\Gamma|=s t, H$ be a subgroup of $\Gamma$ such that $|H|=$ $t$ and $G=t K_{s+1}$. We shall construct a mapping $f \in F(G, \Gamma)$ such that $G$ is not $(\Gamma, f)$-colorable. For each $u, v \in V\left(K_{s+1}\right)$, let $f[u v]=H$. Assume by contradiction that there exists a $(\Gamma, f)$-coloring $c: V(G) \rightarrow \Gamma$.

Claim 1. For any pair of vertices $u, v \in V\left(t K_{s+1}\right), c(u)$ and $c(v)$ must be in different right cosets of $H$ in $\Gamma$.

By way of contradiction, we assume there exists such vertices $u, v \in V\left(t K_{s+1}\right)$ such that $c(u), c(v) \in H g$ for some $g \in \Gamma$. We may assume without loss of generality that each arc in $[u v]$ is oriented towards $v$. It is well known that two cosets of a subgroup are either identical or disjoint since congruence modulo $H$ is an equivalence relation (see, for example, Chapter I, Theorem 4.2 and Corollary 4.3 of [12]). Therefore, since $c(v) \in H g$, we have $H c(v) \cap H g \neq \varnothing$, and so $H c(v)=$ $H g$. As we also have $c(u) \in H g=H c(v)$, it follows that $c(u)[c(v)]^{-1} \in H$. As $f[u v]=H, c$ cannot be a $(\Gamma, f)$-coloring, contrary to the assumption that $c$ is a $(\Gamma, f)$-coloring. This proves Claim 1. 
By Claim 1, for any $u, v \in V\left(t K_{s+1}\right), c(u)$ and $c(v)$ must be in different cosets of $H$ in $\Gamma$. However, as there are $s+1$ vertices and only $s$ cosets, we conclude that such a coloring $c$ does not exist.

Corollary 2.2.19 Let $A$ be an abelian group with $|A|=$ st. Then $t K_{s+1}$ is not A-colorable.

Proof: Since $A$ is abelian and $t$ divides $A$, there must be a subgroup $H$ with $|H|=t$ (see [12] II Corollary 2.4). Corollary 2.2.19 now follows directly from Theorem 2.2.18.

By Corollary 2.2.19 with $s=2$ and $t=6$, we observe that the conclusion of Proposition 2.2.13 can be extended to the form that for every abelian group $A$ with $|A|=12,6 K_{3}$ is not $A$-colorable, as mentioned earlier. Moreover, it is not difficult to see that Theorem 2.2.17 and Theorem 2.2.18 together constitute an infinite and somewhat diverse set of examples for graphs where group structure matters in group coloring. As stated before, the CLT property has not been characterized, but has been otherwise studied. A look at [3] tells us that CLT groups are solvable, a more well-known property than CLT. Therefore, one could create more examples using non-solvable groups.

All of the previous examples in this section have only one of $\Gamma_{1}$ and $\Gamma_{2}$ as a non-abelian group, but this is not a necessary condition for a counter-example to exist. We may note that there is a (nonabelian) dicyclic group $\mathrm{Dic}_{3}$ (also written as $Q_{12}$ ) with order 12 and a subgroup of order 6 . Using virtually the same proof as in Theorem 2.2.18, we see that $6 K_{3}$ is not $\mathrm{Dic}_{3}$-colorable. However, as previously stated, $6 K_{3}$ is $A_{4}$-colorable even though $\left|\operatorname{Dic}_{3}\right|=\left|A_{4}\right|=12$.

\subsubsection{Further Questions}

After answering Question 2.2.2 in the negative, there are some natural first steps toward determining whether $\chi_{a}(G)$ and $\chi_{g}(G)$ can differ. In this last subsection, we state these further questions and discuss them briefly. 
Question 2.2.20 Is there a graph $G$ such that $G$ is $A$-colorable but not $\Gamma$ colorable where $A$ is an abelian group, $\Gamma$ is a non-abelian group, and $|A|=|\Gamma|$ ?

Question 2.2.21 Is there a graph $G$ such that $G$ is $A_{1}$-colorable but not $A_{2}$ colorable where $A_{1}$ and $A_{2}$ are two abelian groups with $\left|A_{1}\right|=\left|A_{2}\right|$ ?

Questions 2.2.20 and 2.2.21 can, like Question 2.2.2, be considered steps toward determining whether $\chi_{a}(G)$ and $\chi_{g}(G)$ can differ. The methods used in this section to answer Question 2.2.2 cannot be easily applied to these further questions since abelian groups are CLT, i.e. they always have subgroups of order $k$ for any $k$ that divides the order of the group (see [12] II Corollary 2.4). Indeed, Corollary 2.2.19 demonstrates that a new approach is needed in answering these further questions. Finally, the goal of all previously mentioned questions is to work towards answering the following:

Question 2.2.22 Does there exist a graph $G$ such that $\chi_{a}(G)<\chi_{g}(G)$ ?

\subsection{Group Coloring and Disjoint Unions}

\subsubsection{Motivation}

The research in this section is motivated by the coloring result proved by Burr in [5], stated as follows:

Theorem 2.3.1 [5] Let $G$ be a simple graph and $m_{1}, m_{2} \ldots m_{k}$ be positive integers. Then $\chi(G) \leq m_{1} m_{2} \ldots m_{k}$ if and only if $G$ is the edge-disjoint union of $k$ graphs $G_{1}, G_{2} \ldots G_{k}$ such that $\chi\left(G_{i}\right) \leq m_{i}$ for $1 \leq i \leq k$.

It is natural to consider to what extent a group coloring version can be justified. For a graph $G$ that is the edge disjoint union of its subgraphs $G_{1}, G_{2} \ldots G_{k}$, we may ask more generally: How else can we describe $\chi_{g}(G)$ using $\chi_{g}\left(G_{1}\right), \chi_{g}\left(G_{2}\right) \ldots \chi_{g}\left(G_{k}\right)$ ? Three separate approaches are explored in the following three subsections. 


\subsubsection{A Partial Analogue of Burr's Theorem}

The proof of Burr's Theorem 2.3.1 found in [5] can be partially adapted to group coloring:

Theorem 2.3.2 If a multigraph $G$ is the edge-disjoint union of $k$ multigraphs $G_{1}, G_{2} \ldots G_{k}$ such that each $G_{i}$ is $\Gamma_{i}$-colorable, then $G$ is $\left(\Gamma_{1} \times \Gamma_{2} \times \ldots \times \Gamma_{k}\right)$ colorable.

Proof: Let $G$ be the edge-disjoint union of $k$ graphs $G_{1}, G_{2} \ldots G_{k}$ such that each $G_{i}$ is $\Gamma_{i}$-colorable. Let $\Gamma=\left(\Gamma_{1} \times \Gamma_{2} \times \ldots \times \Gamma_{k}\right)$ and $f \in F(G, \Gamma)$. To prove the theorem, it is sufficient to find a $(\Gamma, f)$-coloring of $G$. As $\Gamma$ is a product of $k$ groups, $f$ can be viewed as a $k$-tuple $f=\left(f_{1}, f_{2} \ldots f_{k}\right)$, where $f_{i} \in F\left(G, \Gamma_{i}\right)$, for each $i \in\{1,2 \ldots k\}$. Fix an $i$ with $1 \leq i \leq k$. Since each $G_{i}$ is $\Gamma_{i}$-colorable, there exists a $\left(\Gamma_{i}, f_{i}\right)$-coloring $c_{i}: V\left(G_{i}\right) \rightarrow \Gamma_{i}$ of $G_{i}$. Define $c: V(G) \rightarrow \Gamma$ by $c(v)=\left(c_{1}(v), c_{2}(v) \ldots c_{k}(v)\right)$. Since $G$ is the edge-disjoint union of $G_{1}, G_{2} \ldots G_{k}$, for any $e=u v \in E(G)$, there exists a unique $i_{e} \in\{1,2 \ldots k\}$ such that $u v \in E\left(G_{i_{e}}\right)$. It follows that $c_{i_{e}}(u)-c_{i_{e}}(v) \neq f_{i_{e}}(u v)$, and so $c(u)-c(v) \neq f(u v)$ at the $i_{e}$ 's component. It follows that $c$ is a proper $(\Gamma, f)$-coloring of $G$.

However, the converse of Theorem 2.3.2 does not hold, as we will see below.

Proposition 2.3.3 The graph $K_{4,4}$ is $\left(\mathbb{Z}_{2} \times \mathbb{Z}_{2}\right)$-colorable, but cannot be decomposed into two edge-disjoint $\mathbb{Z}_{2}$-colorable subgraphs.

Proof: By Theorem 1.2.2, $\chi_{g}\left(K_{4,4}\right) \leq \Delta\left(K_{4,4}\right)=4$, so that $K_{4,4}$ must be $\left(\mathbb{Z}_{2} \times \mathbb{Z}_{2}\right)$-colorable. Now assume by way of contradiction that $K_{4,4}$ is the edgedisjoint union of two graphs $G_{1}$ and $G_{2}$, both of which are $\mathbb{Z}_{2}$-colorable. By Lemma 1.2.3, $G_{1}$ and $G_{2}$ must both be forests, and thus they each have at most $|V(G)|-1=7$ edges. Then, since we assumed that $K_{4,4}$ is the edge-disjoint union of $G_{1}$ and $G_{2}$, we must have $\left|E\left(K_{4,4}\right)\right| \leq 14$, a contradiction.

Using Theorem 1.2.4, it is simple to construct infinitely many graphs each of which is $\left(\mathbb{Z}_{2} \times \mathbb{Z}_{2}\right)$-colorable, but cannot be decomposed into two edge-disjoint 
$\mathbb{Z}_{2}$-colorable subgraphs. Furthermore, it is important to recall now a property of group colorings that will show us Theorem 2.3.2 is not quite as strong as it appears. Knowing that a graph $G$ is $\Gamma$-colorable does not necessarily imply that $\chi_{g}(G) \leq|\Gamma|$. To see an example of this, albeit in the dual group connectivity form, see [14]. A more thorough treatment of group coloring and group structure is given in Section 2.2 . 


\subsubsection{Forests and Arboricity}

In this subsection we consider extending a standard vertex coloring result involving arboricity (defined in Section 1.1). The result appears in part as an exercise in Section 21.4 of [2]. The proof is omitted, since Theorem 2.3.4 follows from Corollary 2.3.7.

Theorem 2.3.4 For any graph $G$, if $a(G) \leq k$ then $\chi(G) \leq 2 k$.

To work towards a generalization, we revisit the proof using $\bar{\delta}(G)$.

Lemma 2.3.5 If a multigraph $G$ is the edge-disjoint union of $k$ multigraphs $G_{1}, G_{2}, \ldots, G_{k}$ such that $\bar{\delta}\left(G_{i}\right) \leq p_{i}$ for $1 \leq i \leq k$, then $\bar{\delta}(G) \leq\left\lfloor 2\left(1-\frac{1}{|V(G)|}\right) \sum_{i=1}^{k} p_{i}\right\rfloor$.

Proof: Assume that $G$ is the edge-disjoint union of $k$ graphs $G_{1}, G_{2}, \ldots, G_{k}$ such that $\bar{\delta}\left(G_{i}\right) \leq p_{i}$ for $1 \leq i \leq k$. Then $\left|E\left(G_{i}\right)\right| \leq(|V(G)|-1) p_{i}$ and $|E(G)| \leq$ $(|V(G)|-1) \sum_{i=1}^{k} p_{i}$. Likewise, for each subgraph $H$ of $G$ we can get $|E(H)| \leq$ $(|V(H)|-1) \sum_{i=1}^{k} p_{i}$ in the same way, since any such $H$ is also the edge-disjoint union of some $\left\{H_{i}: 1 \leq i \leq k\right\}$ which are subgraphs of $\left\{G_{i}: 1 \leq i \leq k\right\}$, respectively. Now for any subgraph $H$ of $G$ we have the following inequality:

$$
\begin{aligned}
\delta(H) & \leq\left\lfloor\frac{2|E(H)|}{|V(H)|}\right\rfloor \leq\left\lfloor\frac{2(|V(H)|-1)}{|V(H)|} \sum_{i=1}^{k} p_{i}\right\rfloor \\
& =\left\lfloor 2\left(1-\frac{1}{|V(H)|}\right) \sum_{i=1}^{k} p_{i}\right\rfloor \leq\left\lfloor 2\left(1-\frac{1}{|V(G)|}\right) \sum_{i=1}^{k} p_{i}\right\rfloor .
\end{aligned}
$$

This completes the proof.

Since graphs with $\chi_{g}(G)=2$ are so well-characterized by Lemma 1.2.3, we can use Lemma 2.3.5 to further refine Theorem 2.3.2 in the special case where $m_{1}=m_{2}=\ldots m_{k}=2$.

Theorem 2.3.6 If a multigraph $G$ is the edge-disjoint union of $k$ graphs $G_{1}, G_{2} \ldots G_{k}$ such that each $\chi_{g}(G) \leq 2$, then $\chi_{g}(G) \leq\left\lfloor 2 k\left(1-\frac{1}{|V(G)|}\right)\right\rfloor+1$. 
Proof: Assume that $G$ is the edge-disjoint union of $k$ graphs $G_{1}, G_{2} \ldots G_{k}$ such that each $\chi_{g}\left(G_{i}\right) \leq 2$. Then by Theorem 1.2.3, each $G_{i}$ is a forest and thus $\bar{\delta}\left(G_{i}\right) \leq 1$. Now by Lemma 2.3 .5 we have $\bar{\delta}(G) \leq\left\lfloor 2 k\left(1-\frac{1}{|V(G)|}\right)\right\rfloor$, and thus $\chi_{g}(G) \leq\left\lfloor 2 k\left(1-\frac{1}{|V(G)|}\right)\right\rfloor+1$.

The above theorem will seem more familiar when restated in other terms; Corollary 2.3.7 follows directly from Theorem 2.3.6, Lemma 1.2.3, and the definition of arboricity:

Corollary 2.3.7 For any multigraph $G$, if $a(G) \leq k$ then $\chi_{g}(G) \leq\lfloor 2 k(1-$ $\left.\frac{1}{|V(G)|}\right\rfloor+1$.

Ordinarily, when dealing with standard vertex colorings, there is no reason not to simply round down the bound on chromatic number to $2 k$, since $2 k$ will always be smaller than $|V(G)|$ for a minimum covering. However when dealing with multigraphs it is possible for $2 k$ to be greater than $|V(G)|$, so it is important to leave the bound as written to ensure it remains tight for a larger family of multigraphs. We will present a corresponding bound in DP coloring as well as an application of these bounds in Section 4.1.

\subsubsection{Analysis}

In this subsection we attempt to generalize Theorem 2.3.2 to look more like Theorem 2.3.1, while relaxing the conclusion. To do so using methods similar to Section 2.2, a lower bound on $\chi_{g}(G)$ in terms of $\bar{\delta}(G)$ is necessary so we may say $\chi_{g}(G) \leq N$ implies $\bar{\delta}(G) \leq M$ for some $M$ depending only on $N$. It seems that theorems of this form are more difficult than the converse form, since they cannot rely on techniques developed for vertex coloring. We may note that a graph with $\chi(G)=2$ may have an arbitrarily high $\bar{\delta}(G)$. This is not true of group chromatic number, and in general it has been shown that sufficiently high $\bar{\delta}(G)$ will imply that $\chi_{g}(G)$ is as large as required, which is the type of bound that is useful here. 
Theorem 2.3.8 [17] If a graph $G$ contains a subgraph $H$ with $\delta(H) \geq 2$ then $\chi_{g}(G)>\delta(H) / 2 \ln (\delta(H))$

Corollary 2.3.9 For any integer $N \in \mathbb{N}$, there exists an integer $M \in \mathbb{N}$, depending only on $N$, such that $\chi_{g}(G) \leq N$ implies $\bar{\delta}(G)<M$.

Proof: We prove an equivalent statement: For each $N \in \mathbb{N}$ there exists $M \in \mathbb{N}$, depending only on $N$, such that $\bar{\delta}(G) \geq M$ implies $\chi_{g}(G)>N$. By Theorem 2.3.8, $\chi_{g}(G)>\delta(H) / 2 \ln (\delta(H))$ if $\delta(H) \geq 2$ for some subgraph $H$ of $G$. Therefore, since $x / 2 \ln (x)$ is increasing on $(e, \infty)$, we may say that for $\bar{\delta}(G)>e$ we have the following:

$$
\chi_{g}(G)>\max _{H \subseteq G} \frac{\delta(H)}{2 \ln (\delta(H))}=\frac{\bar{\delta}(G)}{2 \ln (\bar{\delta}(G))}
$$

Furthermore since $\lim _{x \rightarrow \infty} x / 2 \ln (x)=\infty$ we may say that sufficiently high $\bar{\delta}(G)$ will ensure that $\chi_{g}(G)$ is as large as required.

Theorem 2.3.10 For any collection $m_{1}, m_{2} \ldots m_{k} \in \mathbb{N}$ there exists $M \in \mathbb{N}$ such that if $G$ is the edge-disjoint union of $k$ graphs $G_{1}, G_{2} \ldots G_{k}$ with $\chi_{g}\left(G_{i}\right) \leq m_{i}$ for $1 \leq i \leq k$ then $\chi_{g}(G) \leq M$

Proof: This follows from Corollary 2.3.9 and Lemma 2.3.5.

Now that the existence of such a bound has been established, it is natural to seek a best bound. We might ask the following question:

Question 2.3.11 What is the smallest integer $a \in \mathbb{N}$ such that if $G$ is the edgedisjoint union of $k$ graphs $G_{1}, G_{2} \ldots G_{k}$ with $\chi_{g}\left(G_{i}\right) \leq m_{i}$ for $1 \leq i \leq k$ then $\chi_{g}(G) \leq a m_{1} m_{2} \ldots m_{k}$ ?

It is clear that $a \geq 1$ since $K_{4}$ can be written as the disjoint union of two paths $T_{1}$ and $T_{2}$, and $\chi_{g}\left(K_{4}\right)=4=\chi_{g}\left(T_{1}\right) \chi_{g}\left(T_{2}\right)$. We are not aware of an example that requires $a>1$, so it may well be that $a=1$ as with Theorem 2.3.1. 


\subsection{Group Chromatic Number of Multigraphs}

\subsubsection{Motivation}

In 1.2.2 we may note that group coloring a multigraph $G$, as opposed to its underlying simple graph $\hat{G}$, becomes a meaningful distinction, unlike classical colorings. This leads naturally to the question: Given a multigraph $G$, and its simplification $\hat{G}$, what is the relationship between $\chi_{g}(G)$ and $\chi_{g}(\hat{G})$ ?

A good way to quantify the difference between $G$ and $\hat{G}$ is the multiplicity of $G$ (written $\mu(G)$ ) which is defined as the cardinality of the largest set of parallel edges in $G$. So a more specific question would be as follows:

Question 2.4.1 Is there a function $f: \mathbb{N}^{2} \rightarrow \mathbb{N}$ such that $\chi_{g}(G) \leq f\left(\mu(G), \chi_{g}(\hat{G})\right)$ ?

In this section we limit our efforts to the most difficult case, multigraphs $G$ with the property that $G=k \hat{G}$ for some $k$, where $|u v|=k$ for each pair of adjacent $u, v \in V(G)$. Any multigraph $G^{\prime}$ can be extended to a graph $G$ of this form by setting $G=\mu\left(G^{\prime}\right) \hat{G}^{\prime}$. Since we then have $G^{\prime}$ as a subgraph of $G$, it is not difficult to see that $\chi_{g}\left(G^{\prime}\right) \leq \chi_{g}(G)$. Therefore any investigation of Question 2.4.1 need only consider this most difficult class of multigraphs.

\subsubsection{A Bound from Multiplicity}

In this subsection we present results in the direction of answering Question 2.4.1. The first result is actually a lower bound, rather than the upper bound requested by Question 2.4.1. The result appears in [26], but the proof here is original.

Theorem 2.4.2 [26] Let $G$ be a simple graph. If $G$ is not $\Gamma$-colorable then $k G$ is not $\left(\mathbb{Z}_{k} \times \Gamma\right)$-colorable.

Proof: Assume $G$ is not $\Gamma$-colorable. Then there exists a function $f \in F(G, \Gamma)$ such that $G$ is not $(\Gamma, f)$-colorable. From $f$ we construct $g \in F\left(k G, \Gamma \times \mathbb{Z}_{k}\right)$ as follows: For each set of parallel edges $\left\{e_{i}: 1 \leq i \leq k\right\}=[u v]$ in $k G$, let $g\left(e_{i}\right)=(f(u v), i)$. Assume by way of contradiction that there exists $c: V(G) \rightarrow$ 
$\Gamma \times \mathbb{Z}_{k}$, a $\left(\Gamma \times \mathbb{Z}_{k}, g\right)$-coloring of $k G$. Then for any adjacent $u, v \in V(G)$ we have $c(u)-c(v) \notin\{f(u v)\} \times \mathbb{Z}_{k}$. If we denote by $c_{1}$ the first index of $c$, then $c_{1}(u)-c_{1}(v) \neq f(u v)$, and thus $c_{1}: V(G) \rightarrow \Gamma$ is a $(\Gamma, f)$-coloring of $G$, a contradiction. Therefore, since there can be no such $\left(\Gamma \times \mathbb{Z}_{k}, g\right)$-coloring $c$ of $k G$, we see that $k G$ is not $\left(\Gamma \times \mathbb{Z}_{k}\right)$-colorable.

Corollary 2.4.3 $\chi_{g}(k G) \geq k\left(\chi_{g}(G)-1\right)+1$ for any simple graph $G$.

Proof: For a simple graph $G$, there exists a group $\Gamma$ with $|\Gamma|=\chi_{g}(G)-1$ such that $G$ is not $\Gamma$-colorable. By Theorem 2.4.2, $k G$ is not $\left(\mathbb{Z}_{k} \times \Gamma\right)$-colorable. Since $\left|\left(\mathbb{Z}_{k} \times \Gamma\right)\right|=k\left(\chi_{g}(G)-1\right)$, we must have $\chi_{g}(k G) \geq k\left(\chi_{g}(G)-1\right)+1$.

Now that the lower bound is established, it is natural to wonder whether it is also an upper bound. So far, we are not aware of a counterexample, so we make the following Conjecture 2.4.4:

Conjecture 2.4.4 $\chi_{g}(k G)=k\left(\chi_{g}(G)-1\right)+1$

The case where $\chi_{g}(G)$ meets the degeneracy bound from Theorem 1.2.1 is quite straightforward.

Theorem 2.4.5 If $\chi_{g}(G)=\bar{\delta}(G)+1$ then $\chi_{g}(k G)=k\left(\chi_{g}(G)-1\right)+1$.

Proof: Assume that $\chi_{g}(G)=\bar{\delta}(G)+1$. By Corollary 2.4.3, it suffices to show that $\chi_{g}(k G) \leq k\left(\chi_{g}(G)-1\right)+1$. Indeed, we have $\chi_{g}(k G) \leq \bar{\delta}(k G)+1=$ $k \bar{\delta}(G)+1=k\left(\chi_{g}(G)-1\right)+1$, so the proof is complete.

However, to even show that a graph $G$ does not meet this degeneracy bound is far from straightforward, and working with $k G$ afterwards can be very challenging. The next subsection examines a partial result of this type, involving even wheels. 


\subsubsection{Coloring Wheels}

In Theorem 4.2.2, it is proven that all Halin graphs other than odd wheels have $\chi_{g}=3$. Furthermore, for any Halin graph $G$, we have $\bar{\delta}(G)=3$, and thus Halin graphs other than odd wheels do not meet the degeneracy bound and seem to be a good class of graphs to test Conjecture 2.4.4. In particular, this subsection concentrates on even wheels, as they seem to be a harder case, compared to non-wheel Halin graphs. Odd wheels are already covered by Theorem 2.4.5.

The first result can be proved using the notation of group coloring but, since it is generalized later by a DP analogue in Theorem 4.3.8, it is listed as a Corollary here.

Corollary 2.4.6 If $W_{n}$ is a wheel where $n$ is even, then $\chi_{g}(k G) \leq 3 k$

To go further, we must first construct some better tools, similar to the snarl (see Definition 2.2.5). The following Proposition 2.4.7 requires much less than the snarl definition (Definition 2.2.5) but still obtains a result similar to Theorem 2.2.10.

Proposition 2.4.7 Let $\Gamma$ be a group, $A, B, F \subseteq \Gamma$ such that $A=\left\{a_{1}, a_{2}\right\}$ and $|B|=|F|=k \geq 2$. If $F^{-1} a_{1}=F^{-1} a_{2}=B$ then $\Gamma$ has a subgroup $H=\left\langle a_{2} a_{1}^{-1}\right\rangle$ such that $|H|>1$ and $|H|$ evenly divides $k$.

Proof: Let $\Gamma, A, B$, and $F$ be as described above and assume that $F^{-1} a_{1}=$ $F^{-1} a_{2}=B$. We also enumerate $B=\left\{b_{1}, b_{2} \ldots b_{k}\right\}$ arbitrarily and then enumerate $F$ more specifically so that $f_{i} a_{1}=b_{i}$ for $i \in[k]$. We may now define a permutation $\sigma \in S_{k}$ by setting, for each $i \in[k], \sigma(i)$ to be the unique index such that $f_{\sigma(i)}^{-1} a_{2}=f_{i}^{-1} a_{1}$. It is well known that any permutation can be decomposed into disjoint cycles (see [12] Chapter I, Theorem 6.3). Let $\left(n_{1}, n_{2} \ldots n_{p}\right)$ be a cycle in such a decomposition of $\sigma$. Then $f_{n_{i}}^{-1} a_{1}=b_{n_{1}}=f_{\sigma\left(n_{1}\right)}^{-1} a_{2}$ for $i \in[p]$, which is to say that $f_{\sigma\left(n_{1}\right)}=f_{n_{i}}\left(a_{2} a_{1}^{-1}\right)$ for $i \in[p]$. Let $h=a_{2} a_{1}^{-1} \in \Gamma$. Then we have $f_{n_{1}}=f_{n_{1}} h^{p}$ and thus $h^{p}=1_{\Gamma}$, and furthermore that $h^{i} \neq 1_{\Gamma}$ for $0<i<p$. Therefore we may note that $H=\langle h\rangle=\left\langle a_{2} a_{1}^{-1}\right\rangle$ is a subgroup of $\Gamma$ with $|H|=p$. 
If $p=k$ (i.e. $\sigma$ is a single cycle) then certainly $|H|$ divides $k$ since $|H|=p=k$. Let us assume instead that we have $p<k$. First we note that for each $i \in[k]$ we must have $\sigma(i) \neq i$, since otherwise $f_{i}^{-1} a_{1}=f_{\sigma(i)}^{-1} a_{1}=f_{i}^{-1} a_{2}$ which implies $a_{1}=a_{2}$, a contradiction. Thus, there are no "trivial" cycles of length 1 . Returning to the cycle we first examined above, we may note that $f_{\sigma(i)}=f_{i}\left(a_{2} a_{1}^{-1}\right)$ for $i \in[k]$ in general, rather than just inside any particular cycle. With this in mind, we conclude that the existence of any cycle of length $q$ in the decomposition of $\sigma$ implies that $h$ has order $q$ in $\Gamma$. Since the order of any element in any group is certainly uniquely determined, it follows that every cycle in the decomposition of $\sigma$ must have length $p$, the same as the first cycle. As these cycles effectively partition $[k]$, and each cycle has length $|H|=p$, it is apparent that $|H|$ evenly divides $k$.

Before we proceed, there is another useful (though again weaker) result similar to Theorem 2.2.10. We will refer to the structure as a snarloid since its definition generalizes Definition 2.2.5, the snarl.

Definition 2.4.8 A (u,v;f)-snarloid is a 3-tuple $\left(L_{u}, L_{v}, f\left(A_{u v}\right)\right)$ with $\left|f\left(A_{u v}\right)\right| \leq$ $\left|L_{u}\right|=\left|L_{v}\right|<2\left|f\left(A_{u v}\right)\right|$ such that $L_{u}, L_{v} \subseteq \Gamma$ and $A_{u v} \subseteq[u v]$ and satisfying the property that for any $a \in L_{u}$, we have $f\left(A_{u v}\right)^{-1} a \subseteq L_{2}$.

To simplify the notation, when $f$ is understood or assumed in the context, we often write $(u, v)$-snarloid rather than $(u, v ; f)$-snarloid. We further define that a $(u, v)$-snarloid with $A_{u v}=[u v]$ is a full width $(u, v)$-snarloid. When $\left|f\left(A_{u v}\right)\right|=\left|L_{u}\right|$ exactly, the snarloid can be more specifically called a snarl, as seen in Definition 2.2.5. In the rest of the subsection, when referencing a $(u, v ; f)$ snarloid, we will often substitute $F$ for $f\left(A_{u v}\right)$.

Proposition 2.4.9 For a given $f \in F(G, \Gamma)$, if there exists a $(u, v)$-snarloid $\left(L_{u}, L_{v}, F\right)$, then each of the following holds.

(i) For any $f \in F$ and $a \in L_{u}$, there exists an element $b \in L_{v}$ such that $f^{-1} a=b$.

(ii) For any $f \in F$ and $b \in L_{v}$, there exists an $a \in L_{u}$ such that $f^{-1} a=b$. 
Proof. Part (i) follows directly from Definition 2.4.8. From the same definition we can see that for any $f \in F$ we have $f^{-1} L_{u} \subseteq L_{v}$. Now, since $\left|f^{-1} L_{u}\right|=\left|L_{u}\right|=$ $\left|L_{v}\right|$, the equality $f^{-1} L_{u}=L_{v}$ must hold for any such $f \in F$. Thus, given $f \in F$ and $b \in L_{v}$ we can specify $a=f b \in f L_{v}=L_{u}$.

Lemma 2.4.10 For a given $f \in F(G, \Gamma)$, if there exists a $(u, v)$-snarloid $\left(L_{u}, L_{v}, F\right)$, then $\left\{b_{i} b_{j}^{-1}: b_{i}, b_{j} \in L_{v}\right\}=\left\{f_{i}^{-1} f_{j}: f_{i} f_{j} \in F\right\}$.

Proof. Let $b_{i}, b_{j} \in L_{v}$. Note that $b_{i}$ and $b_{j}$ can each be written in $F$ different ways (e.g. $b_{i}=f_{j}^{-1} a_{k}$ for some $f_{j} \in F$ and $a_{k} \in L_{u}$ ) using Proposition 2.4 .9 (ii). Since $\left|L_{u}\right|<2|F|$, we must use the same element $a_{r} \in L_{u}$ at least twice while doing so. Thus, we may write $b_{i} b_{j}^{-1}=f_{p}^{-1} a_{r}\left(f_{q}^{-1} a_{r}\right)^{-1}=f_{p}^{-1} a_{r} a_{r}^{-1} f_{q}=f_{p}^{-1} f_{q}$ for some $f_{p}, f_{q} \in F$. Thus, we have $\left\{b_{i} b_{j}^{-1}: b_{i}, b_{j} \in L_{v}\right\} \subseteq\left\{f_{i}^{-1} f_{j}: f_{i} f_{j} \in F\right\}$.

Now, let $f_{i}, f_{j} \in F$ and choose some $a_{k} \in L_{u}$. By Proposition 2.4.9 $(i)$ we way write $f_{i}^{-1} f_{j}=\left(a_{k} b_{p}^{-1}\right)^{-1}\left(a_{k} b_{q}^{-1}\right)=b_{p} a_{k}^{-1} a_{k} b_{q}^{-1}=b_{p} b_{q}^{-1}$ for some $b_{p}, b_{q} \in L_{v}$. Thus, we have $\left\{b_{i} b_{j}^{-1}: b_{i}, b_{j} \in L_{v}\right\} \supseteq\left\{f_{i}^{-1} f_{j}: f_{i} f_{j} \in F\right\}$, completing the proof.

For the remainder of this subsection, when working on a given $f \in F(G, \Gamma)$ and a $(u, v)$-snarloid $\left(L_{u}, L_{v}, F\right)$, we define:

$$
H=\left\{b_{i} b_{j}^{-1}: b_{i}, b_{j} \in L_{v}\right\}=\left\{f_{i}^{-1} f_{j}: f_{i} f_{j} \in F\right\}
$$

Lemma 2.4.11 For a given $f \in F(G, \Gamma)$, if there exists a $(u, v)$-snarloid $\left(L_{u}, L_{v}, F\right)$, then for any $b_{i}, b_{j}, b_{k} \in L_{v}$, there exists $b_{l} \in L_{v}$ such that $b_{i} b_{j}^{-1}=b_{k} b_{l}^{-1}$.

Proof. Let $b_{i}, b_{j}, b_{k} \in L_{v}$. By Lemma 2.4.10, we may write $b_{i} b_{j}^{-1}=f_{x}^{-1} f_{y}$ for some $f_{x}, f_{y} \in F$. Using the given $b_{k}$ and Proposition 2.4.9 (ii), we have $f_{x}=a_{p} b_{k}^{-1}$ for some $a_{p} \in L_{u}$. By Proposition 2.4.9 $(i)$ we now have $f_{y}=a_{p} b_{l}^{-1}$ for some $b_{l} \in L_{v}$. Therefore, $b_{i} b_{j}^{-1}=f_{x}^{-1} f_{y}=\left(a_{p} b_{k}^{-1}\right)^{-1}\left(a_{p} b_{l}^{-1}\right)=b_{k} a_{p}^{-1} a_{p} b_{l}^{-1}=b_{k} b_{l}^{-1}$. 
Lemma 2.4.12 For a given $f \in F(G, \Gamma)$, if there exists a $(u, v)$-snarloid $\left(L_{u}, L_{v}, F\right)$, then $H$ (as defined above) is a subgroup of $\Gamma$.

Proof. Let $h_{1}, h_{2} \in H$. Note that $h_{1} h_{2}^{-1}=\left(b_{p} b_{q}^{-1}\right)\left(b_{j} b_{i}^{-1}\right)^{-1}=b_{p} b_{q}^{-1} b_{i} b_{j}^{-1}$ for some $b_{i}, b_{j}, b_{p}, b_{q} \in L_{v}$. Using Lemma 2.4.11, we see that $b_{i} b_{j}^{-1}=b_{q} b_{l}^{-1}$ for some $b_{l} \in L_{v}$. Therefore $h_{1} h_{2}^{-1}=b_{p} b_{q}^{-1} b_{i} b_{j}^{-1}=b_{p} b_{q}^{-1} b_{q} b_{l}^{-1}=b_{p} b_{l}^{-1} \in H$.

Now that we have established that $H$ is a subgroup of $\Gamma$, we go on to establish $|H|$, so that we can apply Lagrange's Theorem in some coloring arguments later. We can actually prove a stronger property:

Lemma 2.4.13 For a given $f \in F(G, \Gamma)$, if there exists a $(u, v)$-snarloid $\left(L_{u}, L_{v}, F\right)$, then $L_{v}=H b$ for any $b \in L_{v}$. In particular, $|H|=\left|L_{v}\right|$.

Proof. Let $b \in L_{v}$. Recall that $H=\left\{b_{i} b_{j}^{-1}: b_{i}, b_{j} \in L_{v}\right\}$. Thus, for any $b_{i} \in L_{v}$, we may write $b_{i}=b_{i} b^{-1} b \in H b$. This demonstrates that $L_{v} \subseteq H b$. Now, let $b_{j} b_{i}^{-1} b \in H b$. By Lemma 2.4.11 there exists $b_{l} \in L_{v}$ such that $b_{i} b_{j}^{-1}=b b_{l}^{-1}$. Taking an inverse of both sides, we get $b_{j} b_{i}^{-1}=b_{l} b^{-1}$. Thus, we have $b_{j} b_{i}^{-1} b=$ $b_{l} b^{-1} b=b_{l} \in L_{v}$, completing the proof that $L_{v}=H b$, and $|H|=\left|L_{v}\right|$ follows directly.

Now that we have established some tools in Proposition 2.4.7 and Lemma 2.4.13, we can improve the bound from Corollary 2.4.6. The following theorem also gives some (seemingly rare) examples of graphs that are colorable by a smaller group but not colorable by a larger group. Specifically we may note that for odd $n$ we have $\chi_{g}\left(W_{n}\right)=4$ by Theorem 4.2.2. Therefore, since $\operatorname{de} \bar{t} t a\left(W_{n}\right)=3$, we have $\chi_{g}\left(k W_{n}\right)=3 k+1$ by Theorem 2.4.5. However, we will see below that $k W_{n}$ is colorable by any group $\Gamma$ with $|\Gamma|=3 k-1$. For even $n$, the following theorem implies that $\chi_{g}\left(k W_{n}\right) \leq 3 k-1$ (for $k>2$ ). The case where $k=2$ could be handled separately, but would likely fall under another more general proof of a better bound so it has been left for a later time. 
Theorem 2.4.14 Let $n, k \in \mathbb{N}$ such that $n>3$ and $k>2$ and let $\Gamma$ be a group with $|\Gamma|=3 k-1$. Then $k W_{n}$ is $\Gamma$-colorable.

Proof: Let $n, k$, and $\Gamma$ be as described above, and let $f \in F\left(k W_{n}, \Gamma\right)$ be piecewise injective. We will construct a $(\Gamma, f)$-coloring of $k W_{n}$. We label the vertices the same as in previous results with $v_{0}$ at the "hub" and $v_{1}, v_{2} \ldots v_{n}$ in order along the "rim". We begin by coloring $v_{0}$ with some arbitrary $\alpha \in \Gamma$, and then for each $i \in[n]$ we define $L\left(v_{i}\right)=\Gamma-f\left[v_{i} v_{0}\right] \alpha$ to represent the $2 k-1$ choices for $c\left(v_{i}\right)$ that remain when we choose $c\left(v_{0}\right)=\alpha$.

Now, consider that for any $\beta \in L\left(v_{1}\right)$ there are $k$ choices for $c\left(v_{2}\right)$ that are inconsistent with choosing $c\left(v_{1}\right)=\beta$. A poor choice of $\beta$ would have $f\left[v_{1} v_{1}\right] \beta \subset$ $L\left(v_{n}\right)$, leaving only $k-1$ choices for $c\left(v_{n}\right)$ later. If we assume by way of contradiction that every $\beta \in L\left(v_{1}\right)$ is such a poor choice, then $\left(L\left(v_{n}\right), f\left[v_{n} v_{1}\right], L\left(v_{1}\right)\right)$ is a full-width snarloid. Then, by Lemma 2.4.13 $\Gamma$ has a subgroup of order $2 k-1$, which is a contradiction by Lagrange's Theorem (Theorem 2.2.14). Therefore, there must be at least one $\beta \in L\left(v_{1}\right)$ such that $f\left[v_{1} v_{1}\right] \beta \not \subset L\left(v_{n}\right)$ and thus $k$ choices remain for $c\left(v_{n}\right)$ that are consistent with $c\left(v_{0}\right)=\alpha$ and $c\left(v_{1}\right)=\beta$. These $k$ choices remaining for $c\left(v_{n}\right)$ will be denoted by $L^{\prime}\left(v_{n}\right)=L\left(v_{n}\right)-f\left[v_{1} v_{1}\right] \beta=$ $\Gamma-\left[f\left[v_{n} v_{0}\right] \alpha \cup f\left[v_{1} v_{1}\right] \beta\right]$.

Now, starting with $c\left(v_{0}\right)=\alpha$ and $c\left(v_{1}\right)=\beta$, we extend the coloring greedily to $v_{2} \ldots v_{n-1}$. At each step, $c\left(v_{i}\right)$ and $c\left(v_{0}\right)$ may only remove at most $2 k$ choices for $c\left(v_{i+1}\right)$, leaving at least $k-1 \geq 2$ remaining, denoted by $L^{\prime}\left(v_{i+1}\right)$. Assume by way of contradiction that for each $\gamma \in L^{\prime}\left(v_{n-1}\right)$ we have $f\left[v_{n} v_{n-1}\right] \gamma=L^{\prime}\left(v_{n}\right)$ so that no valid choice for $c\left(v_{n}\right)$ remains. Then by Proposition 2.4.7, $\Gamma$ has a nontrivial subgroup $H$ whose order divides $k$. Again, by Lagrange's Theorem and some intuitive number theory, we have a contradiction, since $|H|$ cannot divide both $k$ and $3 k-1$. Thus there is some $\gamma \in L^{\prime}\left(v_{n-1}\right)$ which will allow us to extend the coloring to $v_{n}$, completing the proof. 


\section{Chapter 3}

\section{Group List Coloring}

In this chapter a single result in Group List Coloring is presented. See Subsection 1.2.2 for definitions, basic properties, and previously published results. The result is actually already generalized by Theorem 1.2.15 from [1], but it was proved independently and will be included here to demonstrate another use for the snarl structure (see Definition 2.2.5 and following characterization in Theorem 2.2.10).

\subsection{An Analogue of Brooks' Theorem}

\subsubsection{Motivation}

Brooks' coloring theorem is a well-known classic result in graph coloring theory, which has been extended to group coloring of simple graphs in [23], and to group list coloring of simple graphs in [7].

Theorem 3.1.1 Let $G$ be a simple connected graph.

(i) (Brooks [4], and Theorem 14.4 of [2]) If $G$ is not an odd cycle nor a complete graph, then $\chi(G) \leq \Delta(G)$.

(ii) (Lai and Zhang, Theorem 4.2 of [23]) If $G$ is not a cycle nor a complete graph,

then $\chi_{g}(G) \leq \Delta(G)$. 
(iii) (Chuang et al, Theorem 1.1 of [7]) If $G$ is not a cycle nor a complete graph, then $\chi_{g l}(G) \leq \Delta(G)$.

By definition, for a graph $G$ that permits multiple edges, we have $\chi(G)=\chi(\hat{G})$ and $\chi_{L}(G)=\chi_{L}(\hat{G})$. Unlike classic graph coloring, as shown in [26], it is possible that $\chi_{g}(G)>\chi_{g}(\hat{G})$. The multi-graph version of group coloring Brooks' theorem is proved in [26], in which it is shown that for if a multigraph $G$ with $k=\mu(G)$ and $n=|V(G)|$ is not a $k C_{n}$ nor a $k K_{n}$, then $\chi_{g}(G) \leq \Delta(G)$.

The main result in this section is a multigraph version of group list coloring Brooks' theorem. In the next two subsections, we introduce introduce methods for coloring cycles in multigraphs, and then refine these methods in Subsection 3.1.4 for the main result.

\subsubsection{Coloring Irregular Cycles}

Lemma 3.1.2 Let $G$ be a multigraph. If $\hat{G}=C_{n}$ and there exist adjacent vertices $u, u^{\prime} \in V(G)$ with $d_{G}(u) \neq d_{G}\left(u^{\prime}\right)$, then $G$ is D-group choosable.

Proof. Let $\Gamma$ be a group with $|\Gamma| \geq \Delta(G)$, and $f \in F(G, \Gamma)$. Let $L$ : $V(G) \rightarrow 2^{\Gamma}$ be a $D$-list. As $\hat{G}=C_{n}$, we can label $V(\hat{G})=\left\{v_{i}: i \in \mathbb{Z}_{n}\right\}$ and $E(\hat{G})=\left\{v_{i} v_{i+1}: i \in \mathbb{Z}_{n}\right\}$. By assumption, we may assume and $\left\{u, u^{\prime}\right\}=\left\{v_{1}, v_{2}\right\}$ and that $d_{G}\left(v_{1}\right)<d_{G}\left(v_{2}\right)$. Fix one edge $e_{0} \in\left[v_{1} v_{2}\right]$ and oriented it as $e_{0}=\left(v_{1}, v_{2}\right)$. Orient the edges of $E\left(G-e_{0}\right)$ so that for each $i \in \mathbb{Z}_{n}-\{1\}$, all edges joining $v_{i}$ and $v_{i+1}$ are oriented from $v_{i}$ to $v_{i+1}$, and all edge in $\left[v, v_{2}\right]-\left\{e_{0}\right\}$ are oriented from $v_{2}$ to $v_{1}$. Let $D$ denote the resulting orientation of $G$. We are to construct a $(\Gamma, L, f)$-coloring with $D$.

Since $\left|L\left(v_{2}\right)\right|=d\left(v_{2}\right)>d\left(v_{1}\right)=\left|L\left(v_{1}\right)\right|=\left|\left\{f\left(e_{0}\right)^{-1} g: g \in L\left(v_{1}\right)\right\}\right|$, we set $c\left(v_{2}\right) \in L\left(v_{2}\right)-\left\{f\left(e_{0}\right)^{-1} g: g \in L\left(v_{1}\right)\right\}$. For each $i \in \mathbb{Z}_{n}-\{1, n\}$, assuming that $c\left(v_{i}\right)$ has been defined, set $c\left(v_{i+1}\right) \in L\left(v_{i+1}\right)-\left\{f(e)^{-1} c\left(v_{i}\right): e \in\left[v_{i} v_{i+1}\right]\right\}$. This can be done as $\left|L\left(v_{i+1}\right)\right|=d\left(v_{i+1}\right)=\left|\left[v_{i} v_{i+1}\right]\right|+\left|\left[v_{i+1} v_{i+2}\right]\right|>\left|\left[v_{i} v_{i+1}\right]\right|=$ $\left|\left\{f(e)^{-1} c\left(v_{i}\right): e \in\left[v_{i} v_{i+1}\right]\right\}\right|$. Thus we have chosen $c\left(v_{i}\right) \in L\left(v_{i}\right)$ for all $i \in$ $\mathbb{Z}_{n}-\{1\}$ such that for any $i$ with $2 \leq i \leq n-1$, and for any $e \in\left[v_{i} v_{i+1}\right]$, 
$c\left(v_{i}\right) c\left(v_{i+1}\right) \neq f(e)$. Since $\left|L\left(v_{1}\right)\right|=d\left(v_{1}\right)>\left|\left[v_{n} v_{1}\right]\right|+\left|\left[v_{1} v_{2}\right]-\left\{e_{0}\right\}\right|$, we can define $c\left(v_{1}\right) \in L\left(v_{1}\right)-\left\{f(e)^{-1} c\left(v_{n}\right): e \in\left[v_{n} v_{1}\right]\right\} \cup\left\{f(e)^{-1} c\left(v_{2}\right): e \in\left[v_{1} v_{2}\right]-\left\{e_{0}\right\}\right\}$. By definition, $c$ is a $(\Gamma, L, f)$-coloring under the orientation $D$.

Lemma 3.1.3 Let $k \geq 2$ be an integer and $G$ be a multigraph. If $\hat{G}=K_{n}$, but $G \neq k K n$, then $G$ is D-group choosable.

Proof. Let $G$ be a multigraph such that $\hat{G}=K_{n}$, but $G \neq k K n$. Given any $u, v, w \in V(G)$, the induced subgraph $G[\{u, v, w\}]$ is a triangle with some parallel edges.

If every such triangular induced subgraph were regular, then $G=k K_{n}$. Therefore we may assume that there exist $u, v, w \in V(G)$ such that $G[\{u, v, w\}]$ is not regular. Now, $G[\{u, v, w\}]$ is $D$-group choosable by Lemma 3.1.2, and therefore $G$ is $D$-group choosable by Lemma 1.2.9.

\subsubsection{Coloring Regular Cycles}

To determine $D$-group choosability of regular cyclic multigraphs, we will revisit the concept of a snarl, covered earlier in Subsection 2.2.2. Since the previous definitions were given in the context of group coloring rather than group list coloring, the definitions are results will be restated in this subsection, this time with group list coloring in mind. The proofs will not be included since they do not meaningfully differ from their group coloring counterparts.

Definition 3.1.4 For a given $f \in F(G, \Gamma)$, a given $D$-list $L: V(G) \rightarrow 2^{\Gamma}$, and an edge uv $\in E(G)$, a $(u, v)$-snarl is a 3-tuple $\left(L_{u}, L_{v}, A_{u v}\right)$ with $\left|L_{u}\right|=\left|L_{v}\right|=$ $\left|A_{u v}\right|$ such that $L_{u} \subseteq L(u), L_{v} \subseteq L(v)$ and $A_{u v} \subseteq[u v]$ satisfying the property that

$$
\text { for any } a_{j} \in L_{u}, f\left(A_{u v}\right)^{-1} a_{j}=L_{v} \text {. }
$$

$A(u, v)$-snarl with $A_{u v}=[u v]$ is a full width $(u, v)$-snarl. 
Proposition 3.1.5 If there exists a $(u, v)$-snarl $\left(L_{u}, L_{v}, A_{u v}\right)$, then each of the following holds.

(i) For any $e_{p} \in A_{u v}$ and $a_{q} \in L_{u}$, there exists an element $b_{z} \in L_{v}$ such that $f\left(e_{p}\right)^{-1} a_{q}=b_{z}$.

(ii) For any $a_{p} \in L_{u}$ and $b_{q} \in L_{v}$, there exists an $e_{z} \in A_{u v}$ such that $f\left(e_{z}\right)^{-1} a_{p}=$ $b_{q}$.

(iii) For any $e_{p} \in A_{u v}$ and $b_{q} \in L_{v}$, there exists an $a_{z} \in L_{u}$ such that $f\left(e_{p}\right)^{-1} a_{z}=$ $b_{q}$.

(iv) For any $a_{0} \in L(u)-L_{u}$, we have $\left\{f(e)^{-1} a_{0}: e \in A_{u v}\right\} \cap L_{v}=\varnothing$.

These properties of snarls can be used to prove the following result:

Theorem 3.1.6 If $G$ is a regular multigraph such that $\hat{G}=C_{n}$ but $G \neq k C_{n}$, then $G$ is D-group choosable.

Proof. Let $V(\hat{G})=\left\{v_{i}: i \in \mathbb{Z}_{n}\right\}$ and $E(\hat{G})=\left\{v_{i} v_{i+1}: i \in \mathbb{Z}_{n}\right\}$. Orient the edges of $E(G)$ so that for each $i \in \mathbb{Z}_{n}$, all edges joining $v_{i}$ and $v_{i+1}$ are oriented from $v_{i}$ to $v_{i+1}$. Let $L: V(G) \rightarrow 2^{\Gamma}$ be a $D$-list, and $f \in F(G, \Gamma)$ be an arbitrary mapping. We are to construct a $(\Gamma, L, f)$-coloring $c: V(G) \rightarrow \Gamma$ with $c\left(v_{i}\right) \in L\left(v_{i}\right)$, as follows.

Case 1 For some $t \in \mathbb{Z}_{n}$, there does not exist a full width $\left(v_{t}, v_{t+1}\right)$-snarl.

We may assume that $t=1$. Let $m=\left|v_{1} v_{2}\right|$. Start with an arbitrary color $c\left(v_{n}\right) \in L\left(v_{n}\right)$. For $j=n-1, n-2, \ldots, 3$, define $c\left(v_{j}\right) \in L\left(v_{j}\right)-\left\{f\left(e_{l}\right) c\left(v_{j+1}\right):\right.$ $\left.e_{l} \in\left[v_{j} v_{j+1}\right]\right\}$. Since $d_{G}\left(v_{i}\right)=\left|v_{i-1} v_{i}\right|+\left|v_{i} v_{i+1}\right|$, for any $i \in \mathbb{Z}_{n}$, it follows by the definition of $m$ that there exists subsets $L_{v_{1}} \subseteq L\left(v_{1}\right)-\left\{f(e)^{-1} c\left(v_{n}\right): e \in\left[v_{n} v_{1}\right]\right\}$ and $L_{v_{2}} \subseteq L\left(v_{2}\right)-\left\{f(e) c\left(v_{3}\right): e \in\left[v_{2} v_{3}\right]\right\}$ satisfying $\left|L_{v_{1}}\right|=\left|L_{v_{2}}\right|=m$. Since there is no full width $\left(v_{1}, v_{2}\right)$-snarl, by Definition 3.1.4, there must exist an $a_{p} \in$ $L_{v_{1}}$ such that $\left\{f(e)^{-1} a_{p}: e \in\left[v_{1} v_{2}\right]\right\} \subsetneq\left\{b_{i}: 1 \leq i \leq m\right\}$. Let $c\left(v_{1}\right)=a_{p}$ and choose $c\left(v_{2}\right) \in L_{v_{2}}-\left\{f(e)^{-1} a_{p}: e \in\left[v_{1} v_{2}\right]\right\}$. It is routine to verify that $c$ is a $(\Gamma, L, f)$-coloring. 
Case 2 for each $t \in \mathbb{Z}_{n}$, there exists a full width $\left(v_{t}, v_{t+1}\right)$-snarl.

Since $G$ is regular and $\hat{G}=C_{n}$, for any $i \in \mathbb{Z}_{n}$, we must have $\left|v_{i-1} v_{i}\right|+\left|v_{i} v_{i+1}\right|=$ $\Delta(G)$. Let $m=\left|v_{1} v_{2}\right|$. Since $G \neq k C_{n} m \neq \Delta(G)-m$. Thus for any $i \in \mathbb{Z}_{n}$, $\left|v_{t} v_{t+1}\right|$ equals $m$ or $\Delta(G)-m$ alternately, so $n$ must be even. We may assume, without loss of generality that $m<\Delta(G) / 2$.

By the assumption of Case 2, let $\left(L_{v_{n}}, L_{v_{1}}, A_{v_{n} v_{1}}\right)$ be a $\left(v_{n}, v_{1}\right)$-snarl. We are to construct a $(\Gamma, L, f)$-coloring. Since $m=\left|v_{1} v_{2}\right|$, we have $\left|L_{v_{n}}\right|=\left|L_{v_{1}}\right|=$ $\Delta(G)-m$. As $\Delta(G)-m>m$, we can choose $c\left(v_{n}\right) \in L\left(v_{n}\right)-L_{v_{n}}$. For $j=$ $n-1, n-2, \ldots, 3$, define $c\left(v_{j}\right) \in L\left(v_{j}\right)-\left\{f(e) c\left(v_{j+1}\right): e \in\left[v_{j} v_{j+1}\right]\right\}$. Let $L_{v_{2}}=$ $L\left(v_{2}\right)-\left\{f(e) c\left(v_{3}\right): e \in\left[v_{2} v_{3}\right]\right\}$. Since $m=\left|v_{1} v_{2}\right|$, we have $\left|v_{2} v_{3}\right|=\Delta(G)-m$, and so $\left|L_{v_{2}}\right| \geq \Delta(G)-\left|v_{2} v_{3}\right|=m$. If $\left|L_{v_{2}}\right|>m$ we will replace $L_{v_{2}}$ with an arbitrary subset of size $m$. Since $c\left(v_{n}\right) \in L\left(v_{n}\right)-L_{v_{n}}$, by Proposition 3.1.5(iv), any color in $L_{v_{1}}$ can be a candidate for $c\left(v_{1}\right)$, and similarly any color in $L_{v_{2}}$ can be a candidate for $c\left(v_{2}\right)$.

A pair of colors $\left(\alpha_{1}, \alpha_{2}\right)$ is good if $\alpha_{1} \in L_{v_{1}}$ and $\alpha_{2} \in L_{v_{2}}$ such that $\alpha_{1} \alpha_{2}^{-1} \notin$ $\left\{f(e): e \in\left[v_{1} v_{2}\right]\right\}$. In order to extend $c$ into a $(\Gamma, L, f)$-coloring, it suffices to find a good pair $\left(\alpha_{1}, \alpha_{2}\right)$ and define $c\left(v_{1}\right)=\alpha_{1}$ and $c\left(v_{2}\right)=\alpha_{2}$.

By contradiction, assume that there does not exist any good pairs. Thus for any $a_{p}^{\prime} \in L_{v_{1}}$ we have $\left\{f(e)^{-1} a_{p}^{\prime}: e \in\left[v_{1} v_{2}\right]\right\}=L\left(v_{2}\right)-\left\{f(e) c\left(v_{3}\right): e \in\left[v_{2} v_{3}\right]\right\}$. Therefore, for any fixed $e_{q}^{\prime} \in\left[v_{1} v_{2}\right.$, we have $\left\{f\left(e_{q}^{\prime}\right)^{-1} a_{i}^{\prime} \in L_{v_{1}}\right\} \subseteq L_{v_{2}}$. As $\Delta(G)-$ $m>m$, there would be distinct $a_{r}^{\prime}$ and $a_{s}^{\prime}$ in $L_{v_{n}}$ such that $f\left(e_{q}^{\prime}\right)^{-1} a_{r}^{\prime}=f\left(e_{q}^{\prime}\right)^{-1} a_{s}^{\prime}$, forcing $a_{r}^{\prime}=a_{s}^{\prime}$, a contradiction. Thus there must be a good pair of colors so that $c$ can be extended to a $(\Gamma, L, f)$-coloring of $G$.

Since a $(\Gamma, L, f)$-coloring of $G$ exists in both cases, Theorem 3.1.6 holds.

The concept of a snarl can also be used to construct counterexamples in order to give a lower bound on $\chi_{g l}$. First we will show necessary and sufficient conditions for a snarl to exist, in the context of group list coloring. Again, the proofs of the following results do not significantly differ from their counterparts in Subsection 2.2.2, so their proofs have been omitted here. 
Through the rest of this subsection, we assume that $G$ is a given graph and $L: V(G) \rightarrow 2^{\Gamma}$ is a list assignment, $u v \in E(G)$, and unless otherwise stated, we always use $\left(L_{u}, L_{v}, A_{u v}\right)$ to denote a $(u, v)$-snarl, assuming the existence of it. For a fixed $f \in F(G, \Gamma)$, define $f\left(A_{u v}\right)=\left\{f(e): e \in A_{u v}\right\}$, and define

$$
\begin{aligned}
& H_{1}=\left\{a_{i} a_{j}^{-1}: a_{i}, a_{j} \in L_{u}\right\} \\
& H_{2}=\left\{a_{i}^{-1} a_{j}: a_{i}, a_{j} \in L_{u}\right\} \\
& H_{3}=\left\{b_{i}^{-1} b_{j}: b_{i}, b_{j} \in L_{v}\right\} .
\end{aligned}
$$

Proposition 3.1.7 For a $(u, v)$-snarl $\left(L_{u}, L_{v}, A_{u v}\right)$, each of the following holds.

(i) If $F_{1}=\left\{f_{i} f_{j}^{-1}: f_{i}, f_{j} \in f\left(A_{u v}\right)\right\}$, then $H_{1}=F_{1}$.

(ii) If $F_{2}=\left\{-b_{i} b_{j}: b_{i}, b_{j} \in L_{v}\right\}$, then $H_{2}=F_{2}$.

(iii) If $F_{3}=\left\{-f_{i} f_{j}: f_{i}, f_{j} \in f\left(A_{u v}\right)\right\}$, then $H_{3}=F_{3}$.

(iv) Given any $a_{i}, a_{j}, a_{k} \in L_{u}$, there exists $a_{l} \in L_{u}$ such that $a_{i} a_{j}^{-1}=a_{k} a_{l}^{-1}$.

Lemma 3.1.8 The subsets $H_{1}, H_{2}$, and $H_{3}$ defined in (3.2) are conjugate subgroups of $\Gamma$.

Theorem 3.1.9 Let $G$ be a graph. $\Gamma$ be a group with $|\Gamma| \geq \Delta(G)$. uv $\in E(G)$ and $L: V(G) \rightarrow 2^{\Gamma}$ be a D-list. Let $L_{u}, L_{v}, F \subseteq \Gamma$ be subsets of $\Gamma$ such that $L_{u}=\left\{a_{i}: 1 \leq i \leq m\right\} \subseteq L(u), L_{v}=\left\{b_{i}: 1 \leq i \leq m\right\} \subseteq L(v)$, and for some $A_{u v}=\left\{e_{i}: 1 \leq i \leq m\right\} \subseteq[u v], F=f\left(A_{u v}\right)$. Then the following are equivalent.

(i) $\left(L_{u}, L_{v}, A_{u v}\right)$ is a $(u, v)$-snarl.

(ii) there exist conjugate subgroups $H_{1}, H_{2}$, and $H_{3}$ of $\Gamma$ and elements $a, b, f \in \Gamma$ satisfying $a b^{-1}=f$ such that $L_{u}=H_{1} a=a H_{2}, L_{v}=H_{3} b=b H_{2}$, and $F=$ $H_{1} f=f H_{3}$.

Corollary 3.1.10 Let $G$ be a graph. $\Gamma$ be an abelian group with $|\Gamma| \geq \Delta(G)$. $u v \in E(G)$ and $L: V(G) \rightarrow 2^{\Gamma}$ be a D-list. Let $L_{u}, L_{v}, F \subseteq \Gamma$ be subsets of $\Gamma$ such that $L_{u} \subseteq L(u), L_{\subseteq} L(v)$, and for some $A_{u v} \subseteq[u v], F=f\left(A_{u v}\right)$. Then the following are equivalent.

(i) $\left(L_{u}, L_{v}, A_{u v}\right)$ is a $(u, v)$-snarl.

(ii) there exist a subgroup $H$ of $\Gamma$ and elements $a, b, f \in \Gamma$ satisfying $a b^{-1}=f$ such that $L_{u}=a H, L_{v}=b H$, and $F=f H$. 
The following two lemmas can be proved without the notion of a snarl, specifically by combining Theorem 1.3 of [26] with the knowledge that for any multigraph $G$ we have $\chi_{g}(G) \leq \chi_{g l}(G)$. However, in an effort to demonstrate the use of snarls to construct counterexamples and lower bounds for $\chi_{g l}$, we will use them here now.

Lemma 3.1.11 $\chi_{g l}\left(k C_{n}\right) \geq \chi_{g}\left(k C_{n}\right) \geq 2 k+1$ for any $k$ and $n$.

Proof. Label $V\left(C_{n}\right)=\left\{v_{i}: i \in \mathbb{Z}_{n}\right\}$, and $E\left(C_{n}\right)=\left\{v_{i} v_{i+1}: i \in \mathbb{Z}_{n}\right\}$. Orient the edges of $E(G)$ so that for each $i \in \mathbb{Z}_{n}$, all edges joining $v_{i}$ and $v_{i+1}$ are oriented from $v_{i}$ to $v_{i+1}$. We use $\Gamma=\mathbb{Z}_{2 k}$ as an additive group and construct a mapping $f \in F\left(k C_{n}, \Gamma\right)$ so that there is a full-width snarl at each pair of adjacent vertices. By Corollary 3.1.10, the sufficient and necessary conditions for a snarl to occur would be a subgroup $H$ satisfying Corollary 3.1.10 (ii). In order for the snarl to be full-width, we must have $|H|=k$.

Therefore we may use $H=\left\{g \in \mathbb{Z}_{2 k}:\right.$ and $\left.g \equiv 0(\bmod 2)\right\}$. For each $i \in \mathbb{Z}_{n}-\{n\}$, define $f\left[v_{i}, v_{i+1}\right]=H+1$, and define $f\left[v_{n}, v_{1}\right]=H$. By Definition 3.1.4, if $c\left(v_{1}\right) \in H$ then $c\left(v_{2}\right) \in H$. Similarly, for each $i \in\{1,2, \ldots, n-2\}$, if $c\left(v_{i}\right) \in H$ then $c\left(v_{i+1}\right) \in H$. Thus if $c\left(v_{1}\right) \in H$, then $c\left(v_{n}\right) \in H$. However, if $c\left(v_{n}\right) \in H$, then that $f\left[v_{n}, v_{1}\right]=H$ implies that any $(\Gamma, f)$-coloring $c$ must have $c\left(v_{1}\right) \notin H$, a contradiction. The same proof applies if we start with $c\left(v_{1}\right) \notin H$.

Lemma 3.1.12 $\chi_{g l}\left(k K_{n}\right) \geq \chi_{g}\left(k K_{n}\right) \geq k(n-1)+1$ for any $k$ and $n$.

Proof. We use $\Gamma=\mathbb{Z}_{k(n-1)}$ as an additive group and construct $f \in F\left(k K_{n}, \Gamma\right)$ so that there is a full-width snarl at each pair of adjacent vertices. By Corollary 3.1.10, we set $H=\left\{g \in \mathbb{Z}_{k(n-1)}: g=(n-1) p: p \in \mathbb{Z}_{k}\right\}$. Now, for each $[u, v]$, let $f[u, v]=H$. Then for any $(\Gamma, f)$-coloring $c$, and for any $u, v \in V\left(k K_{n}\right), c(u)$ and $c(v)$ must be in different cosets of $H$ for $c$ to be a coloring. However, there are $n$ vertices and only $n-1$ cosets, so a coloring is impossible. 


\subsubsection{D-Group Choosability and Brooks' Theorem}

Definition 3.1.13 A $\theta$-graph is a simple graph that is a subdivision of $3 K_{2}$. A multigraph $G$ is a generalized $\theta$-graph if the simplification $\hat{G}$ is a $\theta$-graph.

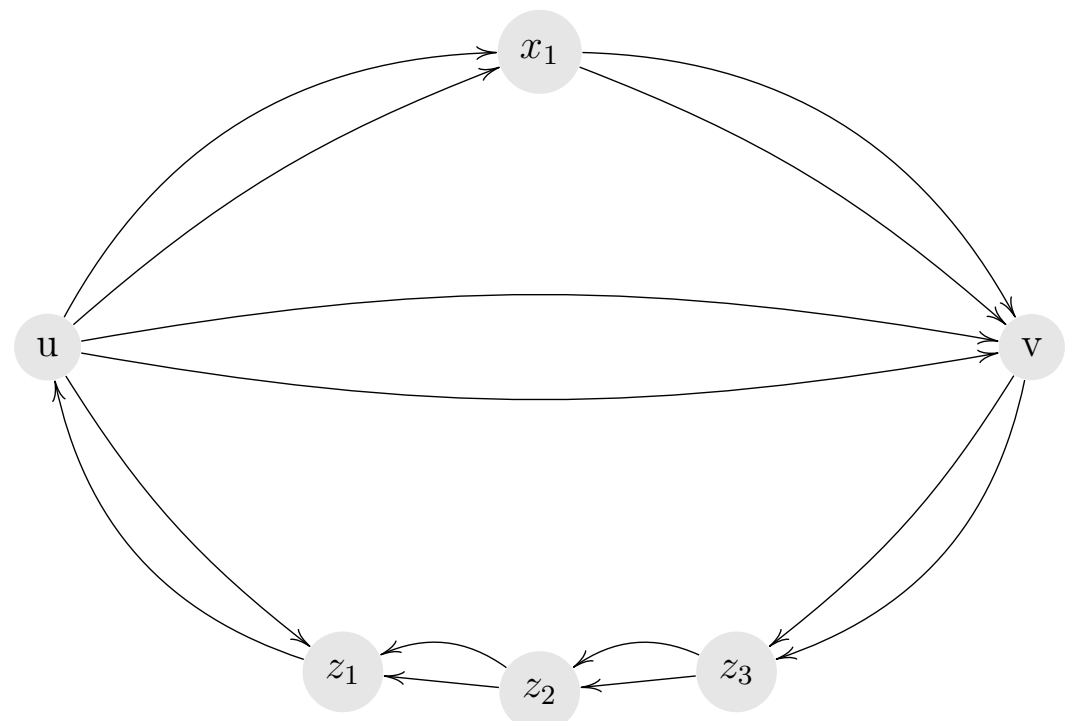

Lemma 3.1.14 Every generalized $\theta$-graph is D-group choosable.

Proof. Let $G$ be a generalized $\theta$-graph. By Definition 3.1.13, there exist two distinct vertices $u, v \in V(G)$ such that $\hat{G}$ consists of three $(u, v)$ paths $P_{1}=$ $x_{0} x_{1} \ldots x_{p}, P_{2}=y_{0} y_{1} \ldots y_{q}$, and $P_{3}=z_{0} z_{1} \ldots z_{r}$ such that $u=x_{0}=y_{0}=z_{0}$ and $v=x_{p}, y_{q}, z_{r}$. By Definition 3.1.13, we assume that $p \geq r>1$. Fix an edge $e_{0} \in\left[u z_{1}\right]$. To show that $G$ is is $D$-group choosable, we assign an orientation $D=D(G)$ by orienting all edges in $\left[x_{i} x_{i+1}\right]$ from $x_{i}$ to $x_{i+1},(0 \leq i \leq p-1)$, all edges in $\left[y_{i} y_{i+1}\right]$ from $y_{i}$ to $y_{i+1},(0 \leq i \leq q-1)$, all edges in $\left[z_{i} z_{i+1}\right]$ from $z_{i+1}$ to $z_{i},(1 \leq i \leq r-1)$ and all edges in $\left[u z_{1}\right]-\left\{e_{0}\right\}$ from $z_{1}$ to $u$. Orient $e_{0}=\left(u, z_{1}\right)$. (See figure for an illustration. )

Suppose first that there exist distinct edges $s_{1} t_{1}$ and $s_{2} t_{2}$ such that $\left|s_{1} t_{1}\right| \neq$ $\left|s_{2} t_{2}\right|$. Then $\hat{G}$ has at least one induced cycle $\hat{C}$ such that $C \neq k C_{n}$. By Lemma 3.1.2 (if $C$ is not regular) or by Theorem 3.1.6 (if $C$ is regular), we conclude that $C$ is $D$-group-choosable. Therefore by Lemma $1.2 .9 G$ is $D$-group-choosable, so we assume that $|u v|=k$ for any pair of adjacent vertices $u, v \in V(G)$. 
Let $\Gamma$ be a group with $|\Gamma| \geq \Delta(G)$, and $f \in F(G, \Gamma)$. Let $L: V(G) \rightarrow 2^{\Gamma}$ be a $D$-list. We are to construct a $(\Gamma, L, f)$-coloring.

Choose $c(u) \in L(u)-\left\{f\left(e_{0}\right)^{-1} g: g \in L\left(z_{1}\right)\right\}$. For each $i \in\{1,2, \ldots, p-1\}$ and $j \in\{1,2, \ldots, q-1\}$, choose $c\left(x_{i}\right) \in L\left(x_{i}\right)-\left\{f(e)^{-1} c\left(x_{i-1}\right): e \in\left[x_{i-1} x_{i}\right]\right\}$ and $c\left(y_{j}\right) \in L\left(y_{j}\right)-\left\{f(e)^{-1} c\left(y_{j-1}\right): e \in\left[y_{j-1} y_{j}\right]\right\}$. Next, we define $c(v) \in$ $L(v)-\left(\left\{f(e)^{-1} c\left(x_{p-1}\right): e \in\left[x_{p-1} v\right]\right\} \cup\left\{f\left(e^{\prime}\right)^{-1} c\left(y_{q-1}\right): e^{\prime} \in\left[y_{q-1} v\right]\right\}\right)$. For the internal vertices of $P_{3}$, we first color, for $i=r-1, r-2, \ldots, 3,2, c\left(z_{i}\right) \in$ $L\left(z_{i}\right)-\left\{f(e)^{-1} c\left(z_{i+1}\right): e \in\left[x_{i} x_{i+1}\right]\right\}$. Finally, we color $c\left(z_{1}\right) \in L\left(z_{1}\right)-(\{f(e) c(u):$ $\left.\left.e \in\left[u z_{1}\right]-e_{0}\right\} \cup\left\{f\left(e^{\prime}\right)^{-1} c\left(z_{2}\right): e^{\prime} \in\left[z_{1} z_{2}\right]\right\}\right)$. It is routine to verify that $c$ is a $(\Gamma, L, f)$-coloring, and thus $G$ is $D$-group choosable.

We now make use of a useful property of $\theta$-graphs, also used in [7] for the simple graph case of Theorem 3.1.16.

Lemma 3.1.15 (Chuang et al. Lemma 2.8 of [7]) Let $G$ be a simple graph with $\kappa(G) \geq 2$. If $G$ is neither a complete graph nor a cycle, then $G$ has an induced $\theta$-subgraph.

The following Theorem 3.1.16 is virtually identical to the simple graph version (Theorem 2.1 of [7]), but has been reproduced here with notation consistent with the rest of this dissertation for the sake of convenience.

Theorem 3.1.16 Let $G$ be a connected multigraph. Then $G$ is D-group choosable if and only if $G$ has a block $B$ such that $B \neq k C_{n}, k K_{n}$.

Proof. Let $G$ be a connected multigraph wtih some block $B \neq k C_{n}, k K_{n}$. Then by Lemma $3.1 .15, \hat{B}$ contains a $\theta$-graph. Therefore, $B$ has an induced subgraph $H$ which is a generalized $\theta$-graph. By Lemma 3.1.14, $H$ is $D$-group choosable, and so by Lemma $1.2 .9, B$ and $G$, are $D$-group choosable as well.

Now, let $G$ be a multigraph such that every block on $G$ is neither $k C_{n}$ nor $k K_{n}$. We proceed by induction on $s$, the number of blocks in $G$. It follows from Lemma 3.1.11 and Lemma 3.1.12 that $G$ is not $D$-group choosable for $s=1$. Now 
let $B_{1}, B_{2} \ldots B_{s}$ be the blocks of $G$. We assume, without loss of generality, that $B_{1}$ is an end block of $G$ (i.e. $B_{1}$ only shares one vertex $v$ with the other blocks). By induction, $K=G-\left(V\left(B_{1}\right)-\{v\}\right)$ is not $D$-group choosable. Then there must exist some group $\Gamma_{1}$ with $\left|\Gamma_{1}\right| \geq \Delta(K)$, some $\left(\Gamma_{1}, D\right)$ list $L_{1}: V(K) \rightarrow 2^{\Gamma_{1}}$, and some $f_{1} \in F\left(K, \Gamma_{1}\right)$ such that $K$ is not $\left(\Gamma_{1}, L_{1}, f_{1}\right)$-colorable. Likewise, since $B_{1}$ is not $D$-group choosable, there is some group $\Gamma_{2}$ with $\left|\Gamma_{2}\right| \geq \Delta\left(B_{1}\right)$, some $\left(\Gamma_{2}, D\right)$ list $L_{2}: V\left(B_{1}\right) \rightarrow 2^{\Gamma_{2}}$, and some $f_{2} \in F\left(B_{1}, \Gamma_{2}\right)$ such that $B_{1}$ is not $\left(\Gamma_{2}, L_{2}, f_{2}\right)$-colorable. Let $\Gamma=\Gamma_{1} \times \Gamma_{2}$. Let $L: V(G) \rightarrow 2^{\Gamma}$ be defined by $L(v)=L_{1}(v) \cup L_{2}(v), L(w)=L_{1}(w)$ for $w \in V\left(G-B_{1}\right.$, and $L(w)=L_{2}(w)$ for $w \in V\left(B_{1}\right)-\{v\}$. Define $f \in F(G, \Gamma)$ by $f(e)=f_{1}(e)$ for $e \in E(K)$ and $f(e)=f_{2}(e)$ for $e \in E\left(B_{1}\right)$. If $G$ has a $(\Gamma, L, f)$ coloring $c$ then there are two possibilities: if $c(v) \in L_{1}(v)$ then $K$ is $\left(\Gamma_{1}, L_{1}, f_{1}\right)$-colorable, and if $c(v) \in L_{2}(v)$ then $B_{1}$ is $\left(\Gamma_{2}, L_{2}, f_{2}\right)$-colorable. Either way there is a contradiction, so we may conclude that $G$ is not $(\Gamma, L, f)$-colorable, and thus not $D$-group choosable.

The main result of this section follows, an analogue of Brook's Theorem:

Theorem 3.1.17 For any connected multigraph $G \cdot \chi_{g l}(G) \leq \Delta(G)+1$, where equality holds if any only if $G$ is a $k C_{n}$ or a $k K_{n}$ for some integers $k \geq 1$ and $n \geq 2$.

Proof. Let $n=|V(G)|$. If $G=k C_{n}$ or $G=k K_{n}$ then $\chi_{g l}(G) \geq \Delta(G)+1$ follows from Lemma 3.1.11 and Lemma 3.1.12 respectively. On the other hand, by Lemma 1.2.8, we have $\chi_{g l}(G) \leq \max _{H \subseteq G}\{\delta(H)\}+1 \leq \Delta(G)+1$. If $G$ is not regular, then $\chi_{g l}(G) \leq \max _{H \subseteq G}\{\delta(H)\}+1 \leq \Delta(G)$. For the remainder of the proof, we will therefore assume that $G$ is regular and that $G$ is neither a $k K_{n}$ nor a $k C_{n}$. We will show that in all remaining cases, $G$ is D-group choosable and thus $\chi_{g l}(G) \leq \Delta(G)$.

Assume first that $\kappa(G) \geq 2$. If $\hat{G} \in\left\{C_{n}, K_{n}\right\}$, then $D$-group choosability of $G$ follows from Theorem 3.1.6 or from Lemma 3.1.3, respectively. If $\hat{G} \notin\left\{C_{n}, K_{n}\right\}$, then by Theorem 3.1.16, $G$ is $D$-group choosable. Therefore, we assume that $G$ is not 2-connected, so $G$ has at least 2 blocks, one of which is an end block $B$. Since 
$G$ is regular, $c \geq 2$ and $B$ is an end block of $G$, it follows that either $\hat{B} \notin\left\{C_{n}, K_{n}\right\}$, whence by Theorem 3.1.16, $B$ is $D$-group choosable; or $\hat{B} \in\left\{C_{n}, K_{n}\right\}$ and $B$ is not regular, and so by Lemmas 3.1 .2 or $3.1 .3, B$ is $D$-group choosable. As $G$ always has an induced subgraph which is $D$-group choosable, it follows by 1.2.9 that $G$ is $D$-group choosable by Theorem 3.1.16. 


\section{Chapter 4}

\section{DP Coloring and $S_{k}$ Coloring}

In this chapter we examine some new results in DP and $S_{k}$ coloring. Most of these results are in some way analogous to results in previous chapters, but one should be careful not to assume that they are direct generalizations since this is often not the case. See Subsections 1.2.3 and 1.2.4 for definitions, basic properties, and some previously published results.

\subsection{DP Coloring and Disjoint Unions}

\subsubsection{Motivation}

As in the group coloring counterpart to this section, The research in this section is motivated by the coloring result proved by Burr in [5], stated as follows:

Theorem 4.1.1 [5] Let $G$ be a simple graph and $m_{1}, m_{2} \ldots m_{k}$ be positive integers. Then $\chi(G) \leq m_{1} m_{2} \ldots m_{k}$ if and only if $G$ is the edge-disjoint union of $k$ graphs $G_{1}, G_{2} \ldots G_{k}$ such that $\chi\left(G_{i}\right) \leq m_{i}$ for $1 \leq i \leq k$.

It is natural to consider to what extent a DP coloring version can be justified. As it turns out, DP coloring seems to be better suited to this type of result, mainly due to Proposition 1.2.11, so we can get a better analogue of Burr's Theorem in DP coloring than in group coloring. Moreover, some alternate approaches from 
Section 2.3 have analogous DP coloring results that will be discussed in the following subsections.

\subsubsection{A Partial DP Coloring Analogue of Burr's Theorem}

We will now attempt another generalization of Theorem 4.1.1, this time to DPcoloring. Just as Theorem 2.3.2 is stated in terms of $\Gamma$-colorability rather than $\chi_{g}$, we will again prove only a partial result, and this time the result is even weaker. We include it here in the interest of examining the natural generalization of Burr's methods to DP-coloring and to introduce the idea of contracting portions of a cover which we will revisit for more interesting results in a future paper. First, we will introduce a specific type of cover.

Definition 4.1.2 Let $G$ be a multigraph, let $m_{1}, m_{2} \in \mathbb{N}$, and let $(L, H)$ be a full $m_{1} m_{2}$-cover of $G$. We say that $(L, H)$ is uniformly $m_{1}$-collapsible if there exist partitions $\left\{L_{1}(v), L_{2}(v), \ldots, L_{m_{1}}(v)\right\}$ for each $v \in V(G)$ such that:

1. $\left|L_{1}(v)\right|=\left|L_{2}(v)\right|=\ldots=\left|L_{m_{1}}(v)\right|=m_{2}$ for each $v \in V(G)$.

2. If $u$ and $v$ are adjacent vertices in $G$, and $i \in\left\{1,2, \ldots, m_{1}\right\}$ then $N_{H}\left(L_{i}(u)\right) \cap$ $L(v)=L_{j}(v)$ for some $j \in\left\{1,2, \ldots, m_{1}\right\}$.

For acyclic graphs, it is not difficult to show that any $m_{1} m_{2}$-cover is uniformly $m_{1}$-collapsible. First, we may designate some arbitrary vertex $v_{0}$ in a given component as a root, then partition $L\left(v_{0}\right)$ appropriately according to the first property of Definition 4.1.2. Now, we partition the neighbors of $v_{0}$ so that the second property of Definition 4.1.2 is satisfied, and continue in this way to neighbors of neighbors until each $L(v)$ is partitioned.

However, for a cycle it is possible to construct an $m_{1} m_{2}$-cover that is not uniformly $m_{1}$-collapsible. The figure below illustrates a 6 -cover for the graph $K_{3}$ that is not uniformly 3-collapsible. To be uniformly 3-collapsible, we would need to partition $L(w)$ into 3 parts of size 2 , but the second property of definition 4.1.2 implies that, in this particular cover, no part may mix elements from inside 
and outside of the set $A$. Therefore, a divisibility argument indicates that $L(w)$ cannot be appropriately partitioned.

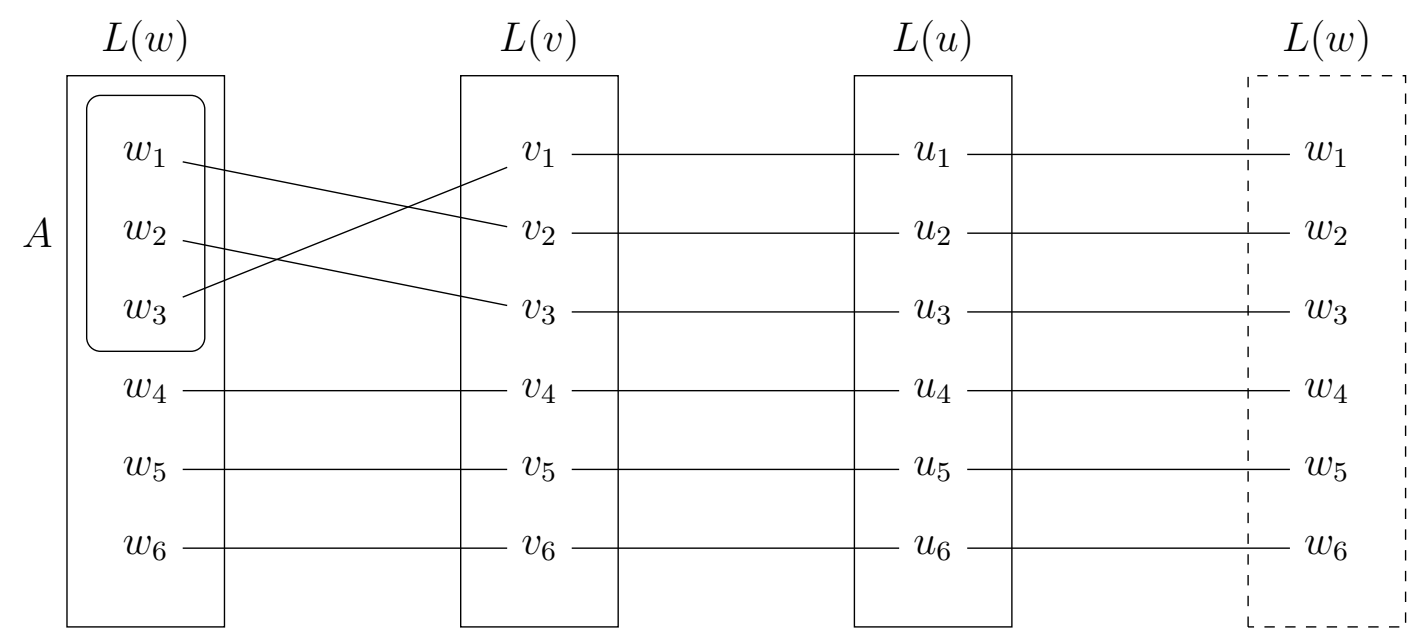

Theorem 4.1.3 Let $G$ be a multigraph, and let $(L, H)$ be a uniformly $m_{1}$-collapsible $m_{1} m_{2}$-cover of $G$. If $G$ is the edge-disjoint union of $G_{1}$ and $G_{2}$ such that $\chi_{D P}\left(G_{i}\right) \leq m_{i}$ for $1 \leq i \leq 2$, then $G$ is $(L, H)$-colorable.

Proof: Let $G,(L, H), G_{1}$ and $G_{2}$ be as described. To prove the theorem, we will construct an $(L, H)$-coloring for $G$. For each $v \in V(G)$, we have an appropriate partition $\left\{L_{1}(v), L_{2}(v), \ldots, L_{m_{1}}(v)\right\}$ of $L(v)$ that satisfies the two properties of Definition 4.1.2. We now modify $(L, H)$ to form a new $m_{1}$-cover $\left(L^{\prime}, H^{\prime}\right)$ as follows: For each $u v \in E\left(G_{2}\right)$, remove $E_{H}[L(u), L(v)]$ from $H$. For each $v \in V(G)$ we identify the vertices in each part $L_{i}(v)$ of the partition, removing any loops and new parallel edges formed, to create the vertex $v_{i} \in V\left(H^{\prime}\right)$. Thus $L^{\prime}(v)=\left\{v_{1}, v_{2}, \ldots, v_{m_{1}}\right\}$ for each $v \in V(G)$. The second property of Definition 4.1.2 implies that $H^{\prime}$ is indeed a cover of $G_{1}$. If we now consider only the edges of $G_{1}$, we may note that $\chi_{D P}\left(G_{1}\right) \leq m_{1}$ implies that there exists an independent set $I^{\prime} \subseteq V\left(H^{\prime}\right)$ with $\left|I^{\prime}\right|=\left|V\left(G_{1}\right)\right|$. We use this set $I^{\prime}$ to construct another graph $H^{\prime \prime}$ from $H$ as follows: Remove all $u \in V(H)$ except those contracted to form the vertices in $I^{\prime}$, and for each $u v \in E\left(G_{1}\right)$ remove $E_{H}[L(u), L(v)]$. We may also 
define, for each $v \in V(G), L^{\prime \prime}(v)=L(v) \cap V\left(H^{\prime \prime}\right)$. Then $\left(L^{\prime \prime}, H^{\prime \prime}\right)$ is an $m_{2}$-cover of $G_{2}$, and $\chi_{D P}\left(G_{2}\right) \leq m_{2}$ implies that there is an independent set $I^{\prime \prime} \subseteq V\left(H^{\prime \prime}\right)$ with $\left|I^{\prime \prime}\right|=\left|V\left(G_{2}\right)\right|=|V(G)|$. We may now note that $I^{\prime \prime}$ is independent in $H$ as well: by the construction of $H^{\prime}$ and $H^{\prime \prime}$, for any two $u, v \in I^{\prime \prime}$ we have $u v \notin E(H)$, since $u v$ cannot cover an edge of $G_{1}$ nor of $G_{2}$. Thus, we have by definition an $(L, H)$-coloring of $G$.

It would be possible to write Theorem 4.1.3 with slightly weaker assumptions; specifically we only need to require that the second property in Definition 4.1.2 holds along edges of $G_{1}$, but for the sake of clarity we elect to leave the theorem as written. Likewise, it is possible to perform an induction to prove a version with more subgraphs in the decomposition, but it would be cumbersome to read due to the collapsibility requirements at each step.

Just as in Theorem 2.3.2, the converse of Theorem 4.1.3 does not hold. Indeed the same graph $K_{4,4}$ serves as a counter-example. By Theorem 1.2.15 (ii), $\chi_{D P}\left(K_{4,4}\right) \leq \Delta\left(K_{4,4}\right)=4$, implying that any uniformly collapsible 4-cover $(L, H)$ of $K_{4,4}$ is colorable. We may be assured that a uniformly collapsible cover exists, as it is evident that the covers equivalent to standard vertex coloring will be uniformly collapsible for any graph. However, as previously demonstrated, $K_{4,4}$ cannot be decomposed into two edge-disjoint forests. Therefore by Corollary 1.2.14, $K_{4,4}$ cannot be decomposed into two graphs $G_{1}$ and $G_{2}$ with $\chi_{D P}\left(G_{1}\right) \leq 2$ and $\chi_{D P}\left(G_{2}\right) \leq 2$.

\subsubsection{Forests and Arboricity}

We now briefly revisit some previous results in group coloring from Section 2.3 and present analogue DP coloring results. As with group coloring, since graphs with $\chi_{D P}(G)=2$ are so well-characterized by Corollary 1.2.14, we can use Lemma 2.3.5 to further refine Theorem 4.1.3 in the special case where $m_{1}=m_{2}=\ldots m_{k}=2$ :

Theorem 4.1.4 If a multigraph $G$ is the edge-disjoint union of $k$ graphs $G_{1}, G_{2} \ldots G_{k}$ such that each $\chi_{D P}(G) \leq 2$, then $\chi_{D P}(G) \leq\left\lfloor 2 k\left(1-\frac{1}{|V(G)|}\right)\right\rfloor+1$. 
Proof: Assume that $G$ is the edge-disjoint union of $k$ graphs $G_{1}, G_{2} \ldots G_{k}$ such that each $\chi_{D P}\left(G_{i}\right) \leq 2$. Then by Theorem 1.2.14, each $G_{i}$ is a forest and thus $\bar{\delta}\left(G_{i}\right) \leq 1$. Now by Lemma 2.3.5 we have $\bar{\delta}(G) \leq\left\lfloor 2 k\left(1-\frac{1}{|V(G)|}\right)\right\rfloor$, and thus $\chi_{D P}(G) \leq\left\lfloor 2 k\left(1-\frac{1}{|V(G)|}\right)\right\rfloor+1$.

The above theorem will seem more familiar when restated in other terms; Corollary 4.1.5 follows directly from Theorem 4.1.4 and the definition of arboricity:

Corollary 4.1.5 For any multigraph $G$, if $a(G) \leq k$ then we have $\chi_{D P}(G) \leq\left\lfloor 2 k\left(1-\frac{1}{|V(G)|}\right)\right\rfloor+1$.

Ordinarily, when dealing with standard vertex colorings, there is no reason not to simply round down the bound on chromatic number to $2 k$, since $2 k$ will always be smaller than $|V(G)|$ for a minimum covering. However when dealing with multigraphs it is possible for $2 k$ to be greater than $|V(G)|$, so it is important to leave the bound as written to ensure it remains tight for a larger family of multigraphs. We may apply this bound in the following application on multigraphs that are slightly larger than complete graphs.

Proposition 4.1.6 Let $p>0$ be an odd number and $G$ be a multigraph of the form $(2 p+1) K_{2 t+1} \cup H$, where $H$ is an acyclic graph with at most $t$ edges. Then $\chi_{g}(G)=\chi_{D P}(G)=2 t(2 p+1)+1$.

Proof: Let $G=(2 p+1) K_{2 t+1} \cup H$, where $H$ is an acyclic graph with at most $t$ edges. First, we demonstrate that $a(G) \leq(p+1)(t+1)$. The subgraph $2 p K_{2 t+1}$ can be covered by $p(2 t+1)$ forests. Next, we cover the remaining edges which induce $K_{2 t+1} \cup H$ as follows: Pick some vertex $v$ that is not on $H$ and remove it from $K_{2 t+1}$ to form $K_{2 t}$. This copy of $K_{2 t}$ can be covered by $t$ trees which also span $K_{2 t}$. Each of these trees can be extended so that it spans $v$ as well by adding edges between $t$ vertices of $K_{2 t}$ and $v$, but we will defer this choice until deciding which edges are appropriate. Consider the graph $H$ on the vertex set 
$V(G)-v$. Since $H$ has at most $t$ edges, it must have at least $t$ components, some of which may be isolated vertices. Select $t$ of these components, and select one vertex from each selected component yielding $\left\{u_{i}: 1 \leq i \leq t\right\}$. We form a forest $F$ by starting with $H$ and drawing the edges $u_{i} v$ for $1 \leq i \leq t$. Finally we return to the choice we deferred earlier and extend our spanning $t$ trees to cover $v$ as well by drawing edges from each $u \in V(G)-\left(v \cup\left\{u_{i}: 1 \leq i \leq t\right\}\right)$ to $v$. Now $K_{2 t+1} \cup H$ is covered by $t+1$ forests, and thus $G$ is covered by $p(2 t+1)+t+1$ forests so $a(G) \leq p(2 t+1)+t+1$.

Now, by Corollary 4.1 .5 and Inequality 1.1 we have $\chi_{g}(G) \leq \chi_{D P}(G) \leq$ $\left\lfloor 2(p(2 t+1)+t+1)\left(1-\frac{1}{2 t+1}\right)\right\rfloor+1=2 t(2 p+1)+1$. By Theorem 1.2.2, $\chi_{g}\left((2 p+1) K_{2 t+1}\right)=\Delta\left((2 p+1) K_{2 t+1}\right)+1=2 t(2 p+1)+1$, and thus $\chi_{g}(G)=$ $\chi_{D P}(G)=2 t(2 p+1)+1$ exactly.

A similar (and much simpler) calculation shows that just as we may add an acyclic graph with $t$ edges to $(2 p+1) K_{2 t+1}$ without increasing the group chromatic number or DP-chromatic number, and so we may say the same about adding an acyclic graph with $t$ edges to $r K_{2 t}$ for any integers $r$ and $t$. In general, the value of $\bar{\delta}(G)$ is not always obvious so for some classes of graphs, like in Proposition 4.1.6, so it may be easier to bound the value of $\bar{\delta}(G)$ with $a(G)$.

\subsubsection{Analysis}

We may adapt the group coloring result in Theorem 2.3.10 using only the fact that $\chi_{g}(G) \leq \chi_{D P}(G)$ for any graph $G$ to obtain the following analogue in DP coloring:

Theorem 4.1.7 For any $m_{1}, m_{2}, \ldots, m_{k} \in \mathbb{N}$ there exists $M \in \mathbb{N}$ such that if $G$ is the edge-disjoint union of $k$ graphs $G_{1}, G_{2}, \ldots, G_{k}$ with $\chi_{D P}\left(G_{i}\right) \leq m_{i}$ for $1 \leq i \leq k$ then $\chi_{D P}(G) \leq M$.

Now that the existence of such a bound has been established, it is natural to seek the best bound. We might ask the following question: 
Question 4.1.8 What is the smallest integer $a \in \mathbb{N}$ such that if $G$ is the edgedisjoint union of $k$ graphs $G_{1}, G_{2}, \ldots, G_{k}$ with $\chi_{D P}\left(G_{i}\right) \leq m_{i}$ for $1 \leq i \leq k$ then $\chi_{D P}(G) \leq a m_{1} m_{2} \ldots m_{k}$ ?

It is clear that, as with group coloring, we have $a \geq 1$ since $K_{4}$ can be written as the disjoint union of two paths $T_{1}$ and $T_{2}$, and $\chi_{D P}\left(K_{4}\right)=4=$ $\chi_{D P}\left(T_{1}\right) \chi_{D P}\left(T_{2}\right)$. We are not aware of an example that requires $a>1$, so it may well be that $a=1$ as with Theorem 4.1.1.

\subsection{Comparing $\chi_{g}(G)$ and $\chi_{D P}(G)$}

\subsubsection{Motivation}

So far in this dissertation, we have seen methods that work just as well for DP-coloring as they do for group coloring. It is important to note that, as with many such generalizations, some properties are lost. In this section, we explore methods and constructions that have been applied to group coloring, but break when applied to DP-coloring. The first, and possibly most important construction that is lost is the dual. Group coloring, from its conception in [14], has been closely tied to group connectivity. This leads us to extend a former group chromatic number result on Halin graphs to its DP-coloring version, for which no duality argument is known. In [17], it is conjectured that for any graph $G, \chi_{L}(G) \leq \chi_{g}(G)$. In [18], Král and Nejedlý first introduced the list group chromatic number $\chi_{g l}(G)$ of a graph $G$. Further studies of $\chi_{g l}(G)$ can be found in $[7,6,21]$, among others. As the invariants $\chi_{L}(G), \chi_{g}(G), \chi_{g l}(G)$ and $\chi_{D P}(G)$ are considered to be closely related, we in this section will also investigate their relationships, and present an example of a graph $G$ such that $\chi_{g}(G)<\chi_{D P}(G)$.

\subsubsection{Halin Graphs}

The first, and possibly most important construction that is lost when generalizing group coloring to DP coloring is the dual. Group coloring, from its conception 
in [14], has been closely tied to group connectivity (see Corollary 1.2.7) and was in fact defined after this dual form. So far, no dual form of DP-coloring is known. The proof of the following Theorem 4.2.2 in [25] relies heavily on group connectivity of the dual graph. A corresponding theorem for DP-coloring would have to use much different methods. We will prove such a corresponding theorem for DP-coloring below.

Definition 4.2.1 A graph $G$ is a Halin Graph if it is the edge-disjoint union of a tree $T$ with at least 4 vertices, none of which has degree 2, and a cycle $C$, constructed by first embedding $T$ on a plane, and then traversing the boundary of $T$ in the clockwise direction connecting each leaf encountered with the next.

Theorem 4.2.2 [25] Let $G$ be a Halin graph. Then $\chi_{g}(G)=4$ if $G$ is isomorphic to an odd wheel and $\chi_{g}(G)=3$ otherwise.

Definition 4.2.3 For a $k$-cover $(L, H)$ of $G$, we say that $\sigma: E(G) \rightarrow S_{k}$ represents $(L, H)$ when, as in Proposition 1.2.16, $G$ is $\sigma$-colorable if and only if $G$ is $(L, H)$-colorable.

Lemma 4.2.4 Given any full $k$-cover $(L, H)$ of a graph $G$ and any acyclic subgraph $T$ of $G$, there exists some $\sigma: E(G) \rightarrow S_{k}$ such that $\sigma$ represents $(L, H)$ and $\sigma_{u v}=1_{S_{k}}$ for each $u v \in E(T)$.

Proof: Let $(L, H)$ be a $k$-cover of a graph $G$, and let $T$ be an acyclic subgraph of $G$. Choose some $v \in V(T)$ and arbitrarily identify $L(v)$ with $[k]$. Now for each neighbor $u$ of $v$ in $T$, we identify $L(u)$ with $[k]$ so that for $i \in[k]$, we assign $i$ to the element of $L(u)$ that is adjacent in $H$ to the element of $L(v)$ which was assigned $i$. Now $\sigma_{u v}(i)=i$ for $i \in[k]$ (i.e. $\sigma_{u v}=1_{S_{k}}$ ). We may repeat this process until we have identified $L(w)$ with $[k]$ for each $w \in V(T)$. Since $T$ is acyclic, there can be no conflict in assigning $[k]$ to any $L(w)$ for $w \in V(T)$. For $w \notin V(T)$, we may arbitrarily assign $[k]$ to $L(w)$. Now $\sigma$ represents $(L, H)$ and $\sigma_{u v}=1_{S_{k}}$ for each $u v \in E(T)$. 
Lemma 4.2.4 is well-suited to Halin graphs, since Halin graphs can be decomposed into a spanning tree and a cycle along the leaves of the spanning tree. We apply it below to prove a theorem analogous to Theorem 4.2.2.

Theorem 4.2.5 Let $G$ be a Halin graph. Then $\chi_{D P}(G)=4$ if $G$ is isomorphic to an odd wheel and $\chi_{D P}(G)=3$ otherwise.

Proof. Let $W_{k}$ denote the wheel graph on $k+1$ vertices. Let $G$ be a Halin graph. We consider the two cases depending on whether $G$ is a wheel or not.

Case 1. $G$ is a wheel. Thus $G=W_{k}$ for some integer $k \geq 3$.

Let $(L, H)$ be a 3-cover of $G$. Without loss of generality, we may assume that $(L, H)$ is full. We label the vertices of $G$ so $v_{0}$ is the "hub" of the wheel and $v_{1}, v_{2}, \ldots, v_{k}$ are placed in order around the "rim". By Lemma 4.2.4, there exists $\sigma: E(G) \rightarrow S_{3}$ such that $\sigma$ represents $(L, H)$ and $\sigma_{v_{0} v_{i}}=1_{S_{3}}$ for each $i \in k$. To prove this case, we prove a stronger property: If $G$ is not $\sigma$-colorable then $\sigma_{u v}=1_{S_{3}}$ for each $u v \in E(G)$, and thus $G$ must be an odd wheel. First, we construct two partial colorings. Let $c_{0}\left(v_{0}\right)=0$ and $c_{0}\left(v_{1}\right)=2$. Let $c_{1}\left(v_{0}\right)=1$ and $c_{1}\left(v_{1}\right)=2$. Note that our choice for $c_{0}\left(v_{1}\right)$ disallows exactly one choice of $c_{0}\left(v_{2}\right)$, so $c_{0}\left(v_{2}\right) \neq \sigma_{v_{1} v_{2}}\left(c_{0}\left(v_{1}\right)\right)$. Similarly, $c_{0}\left(v_{2}\right) \neq \sigma_{v_{0} v_{2}}\left(c_{0}\left(v_{0}\right)\right)$. Therefore there must be at least one choice remaining for $c_{0}\left(v_{2}\right)$. We may continue to extend $c_{0}$ and $c_{1}$ to $v_{3} \ldots v_{k-1}$ in this way without fear of conflict.

Claim: If for some $i$ with $1 \leq i \leq k-1$ our choices of $c_{0}\left(v_{0}\right)$ and $c_{0}\left(v_{i}\right)$ both forbid the same color at $v_{i+1}$ (i.e. $\left.\sigma_{v_{i} v_{i+1}}\left(c_{0}\left(v_{i}\right)\right)=\sigma_{v_{0} v_{i+1}}\left(c_{0}\left(v_{0}\right)\right)\right)$ then $c_{0}$ can be extended to a $\sigma$-coloring of $G$. The same applies to $c_{1}$.

Proof of Claim: First assume that our choices of $c_{0}\left(v_{0}\right)$ and $c_{0}\left(v_{k-1}\right)$ both forbid the same color at $v_{k}$. Then we have two choices remaining for $c_{0}\left(v_{k}\right)$ that are consistent with $c_{0}\left(v_{0}\right), c_{0}\left(v_{1}\right), \ldots, c_{0}\left(v_{k-1}\right)$, if we disregard $\sigma_{v_{k} v_{1}}$. Only one of these two choices may cause conflict with respect to $\sigma_{v_{k} v_{1}}$, so the other must be a $\sigma$-coloring of $G$. Now we note that if for some $i$ with $1 \leq i \leq k-2$ our choices of $c_{0}\left(v_{0}\right)$ and $c_{0}\left(v_{i}\right)$ both forbid the same color at $v_{i+1}$, then the two options for $c_{0}\left(v_{i+1}\right)$ that are consistent with previous $c_{0}\left(v_{0}\right), c_{0}\left(v_{1}\right), \ldots, c_{0}\left(v_{i}\right)$ must yield two options for $c_{0}\left(v_{i+2}\right)$. By induction we see that this reduces to the case where 
$i=k-1$, and thus $c_{0}$ can again be extended to a $\sigma$-coloring of $G$. This completes the proof of the claim.

We therefore assume that the condition of the claim does not hold, so that possible extensions of $c_{0}$ and $c_{1}$ to $v_{2}, v_{3}, \ldots, v_{k}$ are uniquely determined by the colors they first assign to $v_{0}$ and $v_{1}$. Now, $c_{0}\left(v_{0}\right)=0$ implies $c_{0}\left(v_{2}\right) \neq 0$ and $c_{1}\left(v_{0}\right)=1$ implies $c_{1}\left(v_{2}\right) \neq 1$. Therefore, since the condition of the claim does not hold and since $c_{0}\left(v_{1}\right)=c_{1}\left(v_{1}\right)=2$, we must have $\sigma_{v_{1} v_{2}}(2) \neq 0$ and $\sigma_{v_{1} v_{2}}(2) \neq 1$. This leaves $\sigma_{v_{1} v_{2}}(2)=2$. Similar arguments with different colors at $v_{0}$ and $v_{1}$ show that $\sigma_{v_{1} v_{2}}(0)=0$ and $\sigma_{v_{1} v_{2}}(1)=1$ so that $\sigma_{v_{1} v_{2}}=1_{S_{3}}$. We may also repeat the same argument to show that $\sigma_{v_{i} v_{i+1}}=1_{S_{3}}$ for $2 \leq i \leq k-1$. It remains to show that $\sigma_{v_{k} v_{1}}=1_{S_{3}}$.

Now we consider two more partial colorings $c_{3}$ and $c_{4}$ where $c_{3}\left(v_{0}\right)=2$, $c_{3}\left(v_{1}\right)=0, c_{4}\left(v_{0}\right)=2$ and $c_{4}\left(v_{1}\right)=1$. Since $\sigma_{v_{i} v_{i+1}}=1_{S_{3}}$ for $1 \leq i \leq k-1$, we must have:

$$
\begin{cases}c_{3}\left(v_{i}\right)=0 & \text { when } 1 \leq i \leq k \text { and } i \text { is odd, } \\ c_{3}\left(v_{i}\right)=1 & \text { when } 1 \leq i \leq k \text { and } i \text { is even, } \\ c_{4}\left(v_{i}\right)=1 & \text { when } 1 \leq i \leq k \text { and } i \text { is odd, } \\ c_{4}\left(v_{i}\right)=0 & \text { when } 1 \leq i \leq k \text { and } i \text { is even. }\end{cases}
$$

Since we assume that $c_{3}$ and $c_{4}$ are not $\sigma$-colorings, we can see that when $k$ is even we must have $\sigma_{v_{k} v_{1}}(1)=0$ and $\sigma_{v_{k} v_{1}}(0)=1$, so $\sigma_{v_{k} v_{1}}=(01)$. However, we then have $c_{0}\left(v_{1}\right)=2$ and $c_{0}\left(v_{k}\right)=1$ so $c_{0}$ is a $\sigma$-coloring, a contradiction. If instead $k$ is odd then we must have $\sigma_{v_{k} v_{1}}(0)=0$ and $\sigma_{v_{k} v_{1}}(1)=1$ so $\sigma_{v_{k} v_{1}}=1_{S_{3}}$. To summarize, $W_{k}$ is not $\sigma$-colorable if and only if $\sigma_{u v}=1_{S_{3}}$ for each $u v \in E(G)$ and $k$ is odd. Such a $\sigma$-coloring of $G$ would be equivalent to a vertex coloring of an odd wheel with three colors, obviously not possible. Therefore, $\chi_{D P}\left(W_{k}\right)=3$ when $k$ is even, and $\chi_{D P}\left(W_{k}\right)>3$ when $k$ is odd. Since $\chi_{D P} \leq \bar{\delta}\left(W_{k}\right)+1=4$, we must have $\chi_{D P}\left(W_{k}\right)=4$ when $k$ is odd.

Case 2: $G$ is not a wheel.

By definition, $G$ is an edge-disjoint union of a spanning tree $T$ and a cycle $C$ through the leaves of $T$. Let $(L, H)$ be a full 3-cover of $G$, and let $\sigma: E(G) \rightarrow S_{3}$ such that $\sigma$ represents $(L, H)$ and $\sigma_{e}=1_{S_{3}}$ for each $e \in E(T)$. Again, we assume 
by way of contradiction that $G$ is not $\sigma$-colorable.

Let $J=T-V(C)$, and let $w_{2}$ be a leaf of $J$. We label $V(C)$ with $v_{1}, v_{2}, \ldots, v_{k}$ in order such that $v_{2} \ldots v_{t}$ are adjacent to $w_{2}$ in $G$ and $v_{t+1}, \ldots, v_{k}, v_{1}$ are not adjacent to $w_{2}$ in $G$. Let $w_{1}$ be the vertex of $J$ adjacent to $v_{1}$. By the same reasoning as in Case 1, a fixed partial $\sigma$-coloring of $G-\left\{v_{2}, \ldots, v_{k}\right\}$ must uniquely determine any possible extension to $G-\left\{v_{3}, \ldots, v_{k}\right\}$. Thus, if $c$ is a partial $\sigma$-coloring of $G-\left\{v_{2}, \ldots, v_{k}\right\}$, then $\sigma_{v_{1} v_{2}}\left(c\left(v_{1}\right)\right) \neq \sigma_{w_{2} v_{2}}\left(c\left(w_{2}\right)\right)=c\left(w_{2}\right)$. Now we consider a partial $\sigma$-coloring $c_{0}: V(J) \cup\left\{v_{1}\right\} \rightarrow\{1,2,3\}$ with $c_{0}\left(v_{1}\right)=0, c_{0}\left(w_{1}\right)=1$, and $c_{0}\left(w_{2}\right)=0$. Before proceeding, we will demonstrate that such a proper partial coloring exists.

We define $J^{\prime}=G\left[V(J) \cup\left\{v_{1}\right\}\right]$, a subgraph of the tree $T$, so that we have $\sigma_{e}=1_{S_{3}}$ for each $e \in E\left(J^{\prime}\right)$, and thus $c_{0}$ is proper under $\sigma$ if and only if $c_{0}$ is proper when considered as a standard vertex coloring problem. We further define $P$ to be the unique $\left(v_{1}, w_{2}\right)$-path in $J^{\prime}$, and since $v_{1}$ is a leaf of $J^{\prime}$, we may write a $V(P)$ in order as $v_{1}, w_{1}, \ldots, w_{2}$. If the distance between $w_{1}$ and $w_{2}$ on $P$ is odd, then we may color $P$ by alternating 0 and 1 . Note that we may then change the color of $w_{2}$ if necessary, though this is not required for $c_{0}$. If instead the distance between $w_{1}$ and $w_{2}$ is even, define $w_{p}$ to be the unique vertex on $P$ adjacent to $w_{2}$. As before, we color $v_{1}, w_{1}, \ldots, w_{p-1}$ by alternating 0 and 1 , but then let $c_{0}\left(w_{p}\right)=2$. Now we may set $c_{0}\left(w_{2}\right)=0$ as required. In either case, after coloring $P$ it is routine to greedily extend $c_{0}$ to $J^{\prime}$, since $\bar{\delta}\left(J^{\prime}\right)+1 \leq 3$.

Now that this partial coloring is established, we may note that $\sigma_{v_{1} v_{2}}(0) \neq 0$, or else our choice of $c_{0}\left(v_{1}\right)$ and $c_{0}\left(w_{2}\right)$ forbid the same choice for $c_{0}\left(v_{2}\right)$. By similar reasoning as with $c_{0}$, we may construct a partial coloring $c_{1}: V(J) \cup\left\{v_{1}\right\} \rightarrow$ $\{1,2,3\}$ with $c_{1}\left(v_{1}\right)=0, c_{1}\left(w_{1}\right)=2$, and $c_{1}\left(w_{2}\right)=1$, so $\sigma_{v_{1} v_{2}}(0) \neq 1$, and another partial coloring $c_{2}$ with $c_{2}\left(v_{1}\right)=0, c_{2}\left(w_{1}\right)=1$, and $c_{2}\left(w_{2}\right)=2$, so $\sigma_{v_{1} v_{2}}(0) \neq 2$. Since any $\sigma \in S_{3}$ must map 0 , we have a contradiction.

So in the case of Halin graphs, while the dual methods used for group coloring cannot be applied to DP-coloring, the result still holds. 


\subsubsection{An Example where $\chi_{g}(G)<\chi_{D P}(G)$}

We new construct an example graph $G$ such that $\chi_{g}(G)<\chi_{D P}(G)$. Recall from Corollary 1.2.5 that, for any graph $G, \chi_{g}(G)$ is exactly the maximum of all $\chi_{g}(B)$ where $B$ is a clock of $G$. This property is not present in DP coloring, nor in group list coloring, allowing us to recycle an example previously used to demonstrate the possibility that $\chi_{g}(G)<\chi_{g l}(G)$.

Consider the following graph $G$ :

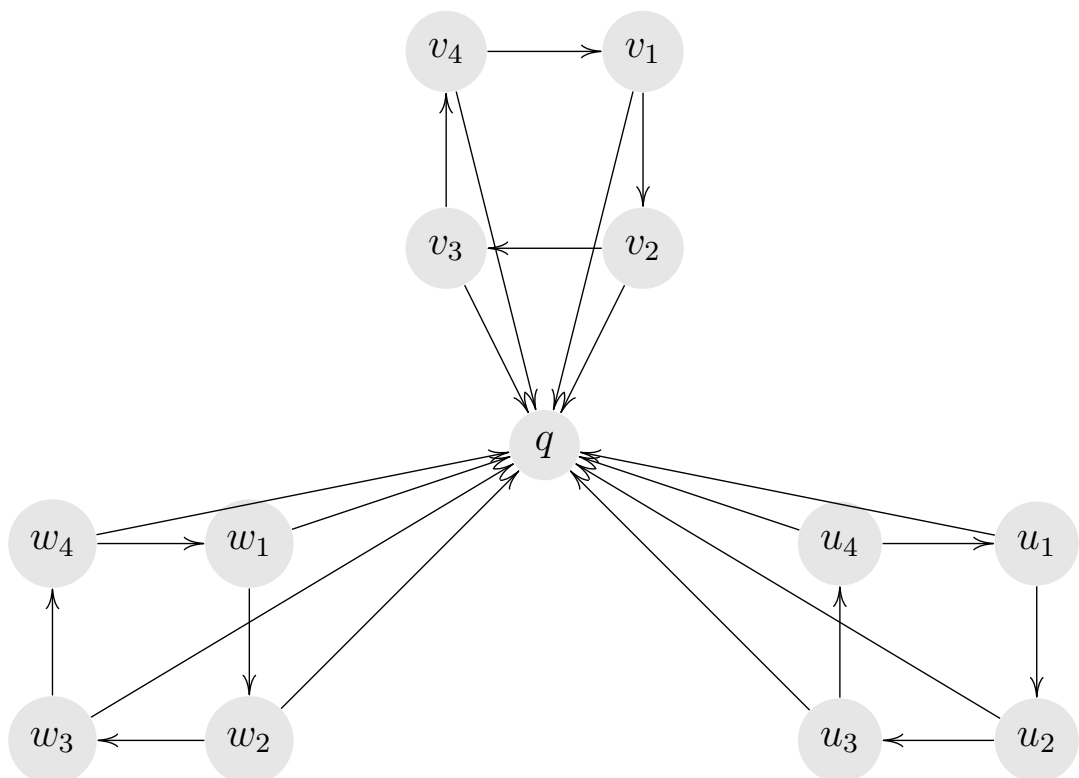

By Theorem 4.2.2 and Corollary 1.2.5 we have $\chi_{g}(G)=3$, but it is proven in [7] that $\chi_{g l}(G)=4$. Therefore, by Proposition 1.2.13 and Lemma 1.2.12 we must also have $\chi_{D P}(G)=4$. Furthermore, we can see that Corollary 1.2.5 does not hold for DP-colorings, since $\chi_{D P}(B)=3$ for each block $B$ of $G$ by Theorem 4.2 .5 .

\subsection{DP Chromatic Number of Multigraphs}

\subsubsection{Motivation}

As in Section 2.4, we may note that DP coloring a multigraph $G$, as opposed to its underlying simple graph $\hat{G}$, becomes a meaningful distinction, unlike classical 
colorings. This leads naturally to the question: Given a multigraph $G$, and its simplification $\hat{G}$, what is the relationship between $\chi_{D P}(G)$ and $\chi_{D P}(\hat{G})$ ? In this section, we show that the results from Section 2.4 have DP coloring analogues and ask the following question:

Question 4.3.1 Is there a function $f: \mathbb{N}^{2} \rightarrow \mathbb{N}$ such that $\chi_{D P}(G) \leq f\left(\mu(G), \chi_{D P}(\hat{G})\right)$ ?

\subsubsection{A Bound From Multiplicity}

In this subsection we present results in the direction of answering Question 4.3.1. The first result is actually a lower bound, rather than the upper bound requested by Question 4.3.1.

Theorem 4.3.2 Let $G$ be a simple graph. If $\chi_{D P}(G)>l$ then $\chi_{D P}(k G)>k l$.

Proof: Assume that $\chi_{D P}(G)>l$. By Proposition 1.2.16 there is a function $\sigma: E(G) \rightarrow S_{l}$ such that $G$ is not $\sigma$-colorable. For each $e=(u, v) \in E(G)$, we enumerate $[u v]=\left\{e_{m}: 1 \leq m \leq k\right\}$. We now construct $\rho: E(k G) \rightarrow S_{[l] \times[k]}$ by $\rho_{e_{m}}(i, j)=\left(\sigma_{e}(i),(j+m)(\bmod \mathrm{k})\right)$. It is not difficult to see that $\rho$ represents a full $k l$-cover of $k G$ which we may denote $(L, H)$. Assume, by way of contradiction that $\chi_{D P}(k G) \leq k l$, so that there is an independent set $S$ of size $|V(G)|$ in $H$. We now construct a cover $(\hat{L}, \hat{H})$ of $G$ as follows: For each $v \in V(G)$ and for each $i \in[l]$, identify the vertices of $H$ labeled by $i \times[k]$ in $L(v)$, removing any resulting loops and multiple edges. Note that $S$ is an independent set in $\hat{H}$, so that $G$ is $(\hat{L}, \hat{H})$-colorable. However, we may note that $\sigma$ represents $(\hat{L}, \hat{H})$. Therefore, since $G$ is not $\sigma$-colorable, $G$ cannot be $(\hat{L}, \hat{H})$-colorable either, a contradiction.

Corollary 4.3.3 $\chi_{D P}(k G) \geq k\left(\chi_{D P}(G)-1\right)+1$

As with group coloring in Section 2.4, it is natural to wonder whether this lower bound is also an upper bound. So far, we are not aware of a counterexample, so we make the following Conjecture 4.3.4: 
Conjecture 4.3.4 $\chi_{g}(k G)=k\left(\chi_{g}(G)-1\right)+1$

Again, the case where $\chi_{D P}(G)$ meets the degeneracy bound from Theorem 1.2.12 is quite straightforward.

Theorem 4.3.5 If $\chi_{D P}(G)=\bar{\delta}(G)+1$ then $\chi_{D P}(k G)=k\left(\chi_{D P}(G)-1\right)+1$.

Proof: Assume that $\chi_{D P}(G)=\bar{\delta}(G)+1$. By Corollary 4.3.3, it suffices to show that $\chi_{D P}(k G) \leq k\left(\chi_{D P}(G)-1\right)+1$. Indeed, we have $\chi_{D P}(k G) \leq \bar{\delta}(k G)+1=$ $k \bar{\delta}(G)+1=k\left(\chi_{D P}(G)-1\right)+1$, so the proof is complete.

As with group coloring, to even show that a graph $G$ does not meet this degeneracy bound is far from straightforward, and working with $k G$ afterwards can be challenging. The next subsection examines a partial result of this type, after establishing some useful notation for future results.

\subsubsection{Coloring Wheels}

In Theorem 4.2.5, it is proven that all Halin graphs other than odd wheels have $\chi_{D P}=3$. Furthermore, for any Halin graph $G$, we have $\bar{\delta}(G)=3$, and thus Halin graphs other than odd wheels do not meet the degeneracy bound and seem to be a good class of graphs to test Conjecture 4.3.4. In particular, this subsection concentrates on even wheels, as they seem to be a harder case, compared to non-wheel Halin graphs. Odd wheels are already covered by Theorem 4.3.5.

Before establishing the main result of this section, a fair amount of notation must be established. First, an adaptation of the definition of a snarl (Definition 2.2.5) to DP coloring:

Definition 4.3.6 Given a multigraph $G$ with $[u v] \subseteq E(G)$ and a full cover $(L, H)$ of $G$, a $(u, v)$-snarl in $(L, H)$ is a triple $(A, B, J)$ with $A \subseteq L(u), B \subseteq L(v)$, $J \subseteq E_{H}[L(u), L(v)]$, and $|A|^{2}=|B|^{2}=|J|$ such that the following two equivalent conditions hold:

(i) For each $v \in A, N_{H[J]}(v)=B$

(ii) $H[J]$ is a complete bipartite graph with bipartition $\{A, B\}$ and edge set $J$ 
We say that a $(u, v)$-snarl $(A, B, J)$ in a full cover $(L, H)$ is a full-width snarl when $|A|=|u v|$. Using Definition 4.3.6 and Definition 4.1.2, it is possible to sometimes reverse the process from Theorem 4.3.2.

Lemma 4.3.7 If $(L, H)$ is a uniformly $k$-collapsible $k l$-cover of $k G$ where $\chi_{D P}(G) \leq$ $l$, then $k G$ is $(L, H)$-colorable.

Proof: Assume that $(L, H)$ is uniformly $k$-collapsible $k l$-cover of $k G$ and $\chi_{D P}(G) \leq l$. Since $(L, H)$ is uniformly $k$-collapsible, there is is a partition $\left.\left\{L_{i}(v): v \in V(k G), 1 \leq i \leq l\right)\right\}$ described in Definition 4.1.2. We now form an $l$-cover $\left(L^{\prime}, H^{\prime}\right)$ of $G$ by contracting each set $L_{i}(v)$ to a single vertex $v_{i}$. To be clear, we have:

$$
\begin{aligned}
L^{\prime}(v) & =\left\{v_{i}: 1 \leq i \leq l\right\} \text { for each } v \in V(G) \\
E\left(H^{\prime}\right) & =\left\{u_{i} v_{j}:\left(L_{i}(u), L_{j}(v), E_{H}\left[L_{i}(u), L_{j}(v)\right]\right) \text { is a }(u, v) \text {-snarl in }(L, H)\right\} \\
& \cup\left\{v_{i} v_{j}: 1 \leq i<j \leq l, v \in V(G)\right\}
\end{aligned}
$$

Since $\chi_{D P}(G) \leq l$, there is an independent set $I^{\prime} \subseteq V\left(H^{\prime}\right)$ with $\left|I^{\prime}\right|=|V(G)|$, an $\left(L^{\prime}, H^{\prime}\right)$-coloring of $G$. We may now construct a set $I$ with $|I|=\left|I^{\prime}\right|=|V(G)|$ by arbitrarily selecting one $x \in L_{i}(v)$ for each $v_{i} \in I^{\prime}$ and note that by Definition 4.1.2(2) we have $I$ independent in $H$. This independent set $I$ shows that $k G$ is $(L, H)$-colorable, and completes the proof.

Definition 4.3.6, Definition 4.1.2, and Lemma 4.3.7 will give us a solid framework to construct useful cases while proving some results involving $\chi_{D P}(k G)$, such as the following Theorem 4.3.8. It is worth noting in Definition 4.1.2 that a uniformly $k$-collapsible $l k$-cover partitions each $E_{H}[L(u), L(v)]$ into $l$ full-width $(u, v)$-snarls. This property will help to show that the cases in Theorem 4.3 .8 are exhaustive. 
Theorem 4.3.8 If $W_{n}$ is a wheel where $n$ is even, then $\chi_{D P}(k G) \leq 3 k$

Proof: Let $(L, H)$ be a full $3 k$-cover of $k W_{n}$. We label the vertices of $W_{n}$ with $v_{0}$ at the "hub" and $v_{1}, v_{2} \ldots v_{n}$ in order along the "rim". To prove the Lemma, it suffices to show that in all cases $k G$ is $(L, H)$-colorable by constructing an appropriate independent set $I$.

Case 1: Assume that $(L, H)$ is uniformly $k$-collapsible. By Lemma 4.1.2 we see that $k W_{n}$ is $(L, H)$-colorable since $\chi_{D P}\left(W_{n}\right)=3$ for even $n$ by Theorem 4.2.5.

Case 2: Assume that for some $v_{l} \in V\left(W_{n}\right)$ with $1 \leq l \leq n$ we have $a \in L\left(v_{l}\right)$ such that for any full-width $\left(v_{l} v_{l+1}\right)$-snarl $(A, B, J)$ in $(L, H)$ we have $a \notin A$. To simplify the notation, we may assume without loss of generality that $l=1$.

First, choose some $u_{0} \in L\left(v_{0}\right)$ such that $u_{0} a \notin E(H)$ and add it to $I$. With this choice, we see that for each $i$ with $1 \leq i \leq n$ we have $k$ fewer options to choose from $L\left(v_{i}\right)$. Indeed, for $1 \leq i \leq n$, let $L^{\prime}\left(v_{i}\right)=L\left(v_{i}\right)-N_{H}\left(u_{0}\right)$ so that $L^{\prime}\left(v_{i}\right)$ will be the set of all $2 k$ remaining options for vertices from $L\left(v_{i}\right)$ which will not conflict with $u_{0}$. By our choice of $u_{0}$, we must have $a \in L^{\prime}\left(v_{1}\right)$. We might add $a$ to $I$ later, but also might need to choose another option.

Now, since $\left|N_{H}(a) \cap L^{\prime}\left(v_{n}\right)\right| \leq k$, we may choose a vertex $u_{n} \in L^{\prime}\left(v_{n}\right)-N_{H}(a)$ that does not conflict with $u_{0}$ or $a$, and add it to $I$ as well. Since $\mid N_{H}\left(u_{n}\right) \cap$ $L^{\prime}\left(v_{1}\right) \mid \leq k$, we may designate a set $S_{1} \subseteq L^{\prime}\left(v_{1}\right)-N_{H}\left(u_{n}\right)$ with $\left|S_{1}\right|=k$ and $a \in S_{1}$. For each $s \in S_{1}$, we may note that $\left\{s, u_{n}, u_{0}\right\}$ is independent in $H$. One of these independent sets will be extended to the required $(L, H)$-coloring of $W_{n}$. First, we define $S_{2}=L^{\prime}\left(v_{2}\right)-\bigcap_{s \in S_{1}} N_{H}(s)$.

Claim: $\left|S_{2}\right|>k$.

If there is some $s \in S_{1}$ with $N_{H}(s) \nsubseteq L^{\prime}\left(v_{2}\right)$, then by definition of $S_{2}$ the claim holds by a counting argument. Otherwise, since $a \in S_{1}$, there is no full-width $\left(v_{1}, v_{2}\right)$ snarl $\left(S_{1}, B, J\right)$ in $(L, H)$, and thus there are distinct $s_{1}, s_{2} \in S_{1}$ with $N_{H}\left(s_{1}\right) \cap L\left(v_{2}\right) \neq N_{H}\left(s_{2}\right) \cap L\left(v_{2}\right)$, and $\left|N_{H}\left(s_{1}\right) \cap L\left(v_{2}\right)\right|=\left|N_{H}\left(s_{2}\right) \cap L\left(v_{2}\right)\right|=k$. Therefore $k<\left|L^{\prime}\left(v_{2}\right)-\left(N_{H}\left(s_{1}\right) \cap N_{H}\left(s_{2}\right)\right)\right| \leq\left|S_{2}\right|$, and the claim holds again. 
Now we may note that until we reach $v_{2}$, there is enough freedom to arbitrarily expand the coloring while traversing the rim of the wheel, starting at $v_{n-1}$ and ending at $v_{3}$ : since $\left|L^{\prime}\left(v_{n-1}\right)-N_{H}\left(u_{n}\right)\right| \geq k$, we select some vertex $u_{n-1}$ from this set of options and add it to to our coloring $I$, and we may repeat this process to greedily select $u_{n-1} \ldots u_{3}$ as needed. Note that $\left|L^{\prime}\left(v_{2}\right)-N_{H}\left(u_{3}\right)\right| \geq k$ so that there is some $u_{2} \in S_{2}-N_{H}\left(u_{3}\right)$. By definition of $S_{2}, u_{2} \in L^{\prime}\left(v_{2}\right)-N_{H}\left(u_{1}\right)$ for some $u_{1} \in S_{1}$. Thus, $I=\left\{u_{0}, u_{1} \ldots u_{n}\right\}$ is the required $(L, H)$-coloring of $W_{n}$.

Case 3: Assume that Case 2 does not apply (and assume again without loss of generality that $l=1)$ but there is some full-width $\left(v_{1}, v_{2}\right)$-snarl $\left(A_{1}, B_{1}, J_{1}\right)$ and some full-width $\left(v_{2}, v_{3}\right)$-snarl $\left(A_{2}, B_{2}, J_{2}\right)$ with $B_{1} \cap A_{2} \neq \varnothing$ and $B_{1} \neq A_{2}$. Since $E_{H}\left[L\left(v_{i}\right), L\left(v_{j}\right)\right]$ is a union of $k$ matchings for any $\left[v_{i} v_{j}\right] \subseteq E\left(k W_{n}\right)$, there can only be at most $k$ instances of $u \in L\left(v_{0}\right)$ with $A_{1} \subseteq N_{H}(u)$, and fewer than $k$ instances where $A_{2}-B_{1} \subseteq N(u)$. Therefore, we may choose $u_{0} \in L\left(v_{0}\right)$ such that $A_{1} \nsubseteq N_{H}\left(u_{0}\right)$ and $A_{2}-B_{1} \nsubseteq N_{H}\left(u_{0}\right)$. Now, as in Case 2 we define $L^{\prime}\left(v_{i}\right)=L\left(v_{i}\right)-N_{H}\left(u_{0}\right)$ so that $L^{\prime}\left(v_{i}\right)$ will be the set of all $2 k$ remaining options for vertices from $L\left(v_{i}\right)$ which will not conflict with $u_{0}$. Then we may choose $u_{1} \in L^{\prime}\left(v_{1}\right) \cap A_{1}, s \in L^{\prime}\left(v_{2}\right) \cap\left(A_{2}-B_{1}\right)$, and $t \in L^{\prime}\left(v_{2}\right)-\left(B_{1} \cup A_{2}\right)$. Note that $\left\{u_{0}, u_{1}, s\right\}$ and $\left\{u_{0}, u_{1}, t\right\}$ are both independent in $H$.

Using this freedom at $v_{2}$, we can show that there is even more freedom at $v_{3}$. Indeed, $N_{H}(s) \cap N_{H}(t) \cap L^{\prime}\left(v_{3}\right)=\varnothing$, since $s \in A_{2}$, part of the full-width $\left(v_{2}, v_{3}\right)$ snarl $\left(A_{2}, B_{2}, J_{2}\right)$ and $t \notin A_{2}$. Thus, every $u \in L^{\prime}\left(v_{3}\right)$ is compatible with either $\left\{u_{0}, u_{1}, s\right\}$ or $\left\{u_{0}, u_{1}, t\right\}$. We may therefore proceed as in Case 2, and expand the coloring while traversing the rim of the wheel, starting at $v_{n-1}$ and ending at $v_{3}$, choosing $u_{i-1} \in L^{\prime}\left(v_{i-1}\right)-N_{H}\left(v_{i}\right)$ for each $i \in\{n, n-1 \ldots 4\}$ as needed. Finally, we choose either $u_{2}=t$ if $u_{3} \in B_{2}$ or $u_{2}=s$ otherwise, so that $I=\left\{u_{0}, u_{2} \ldots u_{n}\right\}$ is the required $(L, H)$-coloring of $W_{n}$.

Case 4: Assume that the "rim" ( $\left.L \uparrow_{V\left(W_{n}\right)-v_{0}}, H-L\left(v_{0}\right)\right)$ is uniformly $k$-collapsible, as is each individual "spoke" $\left.\left(L \Upsilon_{\left\{v_{0}, v_{i}\right\}}\right), H\left[L\left(v_{0}\right) \cup L\left(v_{i}\right)\right]\right)$ for $1 \leq i \leq n$, but 
$(L, H)$ is not uniformly $k$-collapsible as a whole. Since the "rim" is uniformly $k$ collapsible, we can partition each $L\left(v_{i}\right)$ with $1 \leq i \leq n$ into $\left\{L_{1}\left(v_{i}\right), L_{2}\left(v_{i}\right), L_{3}\left(v_{i}\right)\right\}$ as described in Definition 4.1.2. Since $(L, H)$ is not uniformly $k$-collapsible, there must be some $u \in L\left(v_{0}\right), v_{i}$ with $1 \leq i \leq n$, and $L_{l}\left(v_{i}\right)$ with $1 \leq l \leq 3$ such that $N_{H}(u) \cap L\left(v_{i}\right) \neq L_{l}\left(v_{i}\right)$ and $N_{H}(u) \cap L_{l}\left(v_{i}\right) \neq \varnothing$. We will say then that $v_{i}$ is split by $u$. Choose such a $u_{0} \in L\left(v_{0}\right)$ that splits at least one $v_{i}$, and define $L^{\prime}\left(v_{i}\right)=L\left(v_{i}\right)-N_{H}\left(u_{0}\right)$ and $L_{l}^{\prime}\left(v_{i}\right)=L_{l}\left(v_{i}\right)-N_{H}\left(u_{0}\right)$ for each $l$ and $i$. Note that for any $v_{i}$ split by $u_{0}$ we have $L_{l}^{\prime}\left(v_{i}\right) \neq \varnothing$ for $1 \leq l \leq 3$.

If every $v_{i}$ for $1 \leq i \leq n$ is split by $u_{0}$, then there is an $\left(L^{\prime}, H-L\left(v_{0}\right)\right)$-coloring $I^{\prime}$ of the rim vertices by Lemma 4.3.7 and Theorem 1.2.12. Since $u_{0}$ splits all $v_{i}$, this coloring does not conflict with $u_{0}$, and thus $I=I^{\prime} \cup u_{0}$ is an $(L, H)$-coloring of $k W_{n}$.

If there is some $v_{i}$ on the rim that is not split by $u_{0}$, so that $L_{l}^{\prime}\left(v_{i}\right)=\varnothing$ for some $i$ and $l$, then we may assume without loss of generality that $i=1, l=1$, and that $v_{2}$ is split by $u_{0}$. We may now arbitrarily select $u_{1} \in L_{2}^{\prime}\left(v_{1}\right)$, and greedily color $v_{n}, v_{n-1} \ldots v_{3}$ by selecting $u_{n} \in L^{\prime}\left(v_{n}\right)-N_{H}\left(u_{1}\right), u_{n-1} \in L^{\prime}\left(v_{n-1}\right)-N_{H}\left(u_{n}\right)$, etc. Since the rim is uniformly $k$-collapsible, we have $N_{H}\left(u_{1}\right) \cap L^{\prime}\left(v_{2}\right) \subseteq L_{p}^{\prime}\left(v_{2}\right)$ and $N_{H}\left(u_{3}\right) \cap L^{\prime}\left(v_{2}\right) \subseteq L_{q}^{\prime}\left(v_{2}\right)$ for some $p, q \in\{1,2,3\}$. Thus, we may complete the $(L, H)$-coloring by selecting $u_{2} \in L_{r}^{\prime}\left(v_{2}\right) \neq \varnothing$, where $r \in\{1,2,3\}-\{p, q\}$.

Case 5: Assume again that the rim is uniformly $k$-collapsible, but that there is some spoke $\left(\left.L\right|_{\left\{v_{0}, v_{i}\right\}}, H\left[L\left(v_{0}\right) \cup L\left(v_{i}\right)\right]\right)$ that is not. Since the "rim" is uniformly $k$ collapsible, we can partition each $L\left(v_{i}\right)$ with $1 \leq i \leq n$ into $\left\{L_{1}\left(v_{i}\right), L_{2}\left(v_{i}\right), L_{3}\left(v_{i}\right)\right\}$ as described in Definition 4.1.2. Since the spoke is not uniformly $k$-collapsible, and $E_{H}\left[L\left(v_{0}\right), L\left(v_{i}\right)\right]$ is the union of $k$ matchings, there can only be at most $k$ elements $u \in L\left(v_{0}\right)$ that do not split $v_{i}$. Therefore, we may choose some $u_{0} \in L\left(v_{0}\right)$ that splits $v_{i}$ and proceed exactly as in Case 4 to build an $(L, H)$ coloring $I$ of $k W_{n}$.

Since a uniformly $k$-collapsible $3 k$-cover partitions each $E_{H}[L(u), L(v)]$ into 3 full-width $(u, v)$-snarls, the cases above are exhaustive, so the proof is complete 


\section{Chapter 5}

\section{Appendices}

\subsection{Appendix: Proof of Lemma 2.2.16}

Notation: For a group $G$, we denote the commutator subgroup $G^{\prime}=\left\{a b a^{-1} b^{-1}\right.$ : $a, b \in G\}$, and the center of a group $C(G)=\left\{a \in G: g a g^{-1}=a\right.$ for each $\left.g \in G\right\}$.

Lemma 5.1.1 Let $N \triangleleft G$ such that $N \cap G^{\prime}=\{1\}$. Then $N \subseteq C(G)$.

Proof. Let $n \in N$ and $g \in G$. Then $g n g^{-1} n^{-1}=n^{\prime} n^{-1} \in N$ (where $n^{\prime} \in N$ ). By definition, $g n g^{-1} n^{-1} \in G^{\prime}$ as well. Since $N \cap G^{\prime}=\{1\}$, we must have $g n g^{-1} n^{-1}=1$ so that $g n g^{-1}=n$ and thus $n \in C(G)$.

Lemma 5.1.2 $A_{n}$ is the only nontrivial normal subgroup of $S_{n}$ for $n \geq 5$.

Proof. Assume by way of contradiction that $N$ is a nontrivial normal subgroup of $S_{n}$ and $N \neq A_{n}$. Then $N \cap A_{n} \triangleleft A_{n}$. Since $A_{n}$ is simple for $n \neq 4$ (see Hungerford's Algebra Chapter I Theorem 6.10) we must have either $N \cap A_{n}=\{1\}$ or $A_{n} \triangleleft N \triangleleft S_{n}$. Since $\left[S_{n}: A_{n}\right]=2$ we cannot have $A_{n} \triangleleft N \triangleleft S_{n}$. Therefore we may assume that $N \cap A_{n}=\{1\}$. In this case, we first note that $A_{n}=S_{n}^{\prime}$. Now by Lemma 5.1.1, we have $N \subseteq C\left(S_{n}\right)=\{1\}$, a contradiction. 
Lemma 5.1.3 For each $n \geq 5$, the symmetric group $S_{n}$ has no subgroup $H$ with $2<\left[S_{n}: H\right]<n$.

Proof. Let $H$ be a non-trivial subgroup of $S_{n}$. To complete the proof, it suffices to show that $\left[S_{n}: H\right]=2$. First, we note that $G$ acts on the set $S_{n} / H$ of all left cosets of $H$ in $S_{n}$ by left translation. This group action induces a homomorphism $f: S_{n} \rightarrow A\left(S_{n} / H\right)$, where $A\left(S_{n} / H\right)$ is the group of all permutations on the set $S_{n} / H$, and furthermore, $\operatorname{ker}(f) \subseteq H$ (see Hungerford's Algebra Chapter II Theorem 4.5 and Proposition 4.8). Now, $k e r(f)$ is a normal subgroup of $S_{n}$. Since $H$ is nontrivial, $f$ cannot be injective, and thus $\operatorname{ker}(f) \neq 1$. Certainly $\operatorname{ker}(f) \neq S_{n}$ either, again since $H$ is nontrivial. Therefore, by Lemma 5.1.2, we must have $\operatorname{ker}(f)=A_{n}$. Since $\left[S_{n}: \operatorname{ker}(f)\right]=\left[S_{n}: A_{n}\right]=2$ and $\operatorname{ker}(f) \subseteq H$, we must have $\left[S_{n}: H\right] \geq 2$. Since $H$ is nontrivial, $\left[S_{n}: H\right]>1$. Therefore, $\left[S_{n}: H\right]=2$. 


\section{Bibliography}

[1] A. Yu. Bernshteyn, A. V. Kostochka, and S. P. Pron: On DP-Coloring Of Graphs and Multigraphs. Siberian Mathematical Journal 58 (2017) 28-36

[2] J. A. Bondy and U. S. R. Murty, Graph theory, Springer, New York, 2008.

[3] H. G. Bray, A note on CLT groups, Pacific Journal of Mathematics 27 (1968) 229-231.

[4] R. Brooks, On colouring the nodes of a network, Proc. Cambridge Phil. Soc. 37(1941) 194-197.

[5] S. A. Burr, A Ramsey-Theoretic Result Involving Chromatic Numbers, Journal of Graph Theory, 4, 241-242 (1980).

[6] H. Chuang, H.-J. Lai, G.R. Omidi, Keke Wang and N. Zakeri, On group choosability of graphs, II, Graphs and Combinatorics, 30 (2014) 549-563.

[7] H. Chuang, H.-J. Lai, G. R. Omidi, and N. Zakeri, On Group Choosability of Graphs, Ars Combinatoria, 126 (2016) 195-209.

[8] Z. Dvořák, L. Postle: Correspondence Coloring and its Application to ListColoring Planar Graphs Without Cycles of Lengths 4 to 8. Journal of Combinatorial Theory, Series B 129 (2018) 38-54.

[9] P. Franklin: A Six Color Problem. Journal of Mathematics and Physics, 13 (1934) 363-369 
[10] H. Hadwiger: Ber eine Klassifikation der Streckenkomplexe Vierteljahrsschr. Naturforsch. Ges. Zrich., 88 (1943) 133-142

[11] P. J. Heawood: Map colouring theorems, Quarterly J. Math. Oxford Ser., 24 (1890) 322-339

[12] T. W. Hungerford, Algebra. Springer, New York, 2000.

[13] R. Hǔsek, L. Mohelníková, R. Šámal, Group connectivity $\mathbb{Z}_{4}$ vs $\mathbb{Z}_{2}^{2}$, J Graph Theory. 2019;1C11. https://doi.org/10.1002/jgt.22488.

[14] F. Jaeger, N. Linial, C. Payan, and M. Tarsi, Group Connectivity of GraphsA Nonhomogeneous Analogue of Nowhere-Zero Flow Properties, J. Combin. Theory Series B 56 (1992), 165-182.

[15] L. Jin, T.-L. Wong, X. Zhu, Colouring of Generalized Signed Planar Graphs, Discrete Mathematics 342 (2019) 836-843

[16] D. Král: Group Coloring is $\Pi_{2}^{P}$-Complete, Theoretical Computer Science, 349 (2005), 99-111

[17] D. Král, O. Pangrac, H.-J. Voss: A Note on Group Colorings. Journal of Graph Theory, 50 (2005) 123-129.

[18] D. Král, and P. Nejedly: Group Coloring and List Group Coloring Are $\Pi_{2}^{P}$-Complete, Lecture Notes in Computer Science, 3153 (2004) 274-287, Springer-Verlag

[19] H.-J. Lai, X. Li, Y. Shao, and M. Zhan, Group Connectivity and Group Colorings of Graphs - A Survey, Acta Mathematica Sinica 27 (2011), 405434.

[20] H.-J. Lai, X. Li, G. Yu: An Inequality for the Group Chromatic Number of a Graph, Discrete Math., 307 (2007) 3076-3080

[21] H.-J. Lai, G.R. Omidi and G. Raeisi, On group choosability of total graphs, Graphs and Combinatorics, 29 (2013), 585-597. 
[22] H.-J. Lai and X. Li: Group Chromatic number of graph, Graphs and Combinatorics, 21 (2005) 469-474

[23] H.-J. Lai and X. Zhang: Group Colorability of Graphs. Ars Combinatoria, 62 (2002) 299-317.

[24] H.-J. Lai and X. Zhang: Group Colorability of Graphs Without $K_{5}$-Minors, Graphs and Combinatorics, 18 (2002), 147-154.

[25] X. Li: Group Chromatic Number of Halin Graphs Graphs and Combinatorics, 31 (2015), 1531-1538.

[26] H. Li and H. Lai, Group Colorability of Multigraphs, Discrete Mathematics, 313 (2013) 101-104.

[27] E. A. Nordhaus, J. W. Gaddum: On Complementary Graphs, Amer. Math. Monthly, 63 (1956), 175-177

[28] G. Ringel, J. W. T. Youngs: Solution of the Heawood Map-Coloring Problem Proceedings of the National Academy of Sciences, 60 (1968) 438-445

[29] J. J. Rotman, An Introduction to the Theory of Groups, New York, 1999.

[30] G. Szekeres, H.S. Wilf: An Inequality for the Chromatic Number of a Graph, J. Combin. Theory 4 (1968) 1-3. 